\title{
Computation of the unipotent Albanese map on elliptic and hyperelliptic curves
}

\author{
Jamie Beacom ${ }^{1}$ \\ Received: 19 October 2018 / Accepted: 3 December 2019 / Published online: 26 December 2019 \\ (c) The Author(s) 2019
}

\begin{abstract}
We study the unipotent Albanese map appearing in the non-abelian Chabauty method of Minhyong Kim. In particular we explore the explicit computation of the $p$-adic de Rham period map $j_{n}^{d r}$ on elliptic and hyperelliptic curves over number fields via their universal unipotent connections $\mathscr{U}$. Several algorithms forming part of the computation of finite level versions $j_{n}^{d r}$ of the unipotent Albanese maps are presented. The computation of the logarithmic extension of $\mathscr{U}$ in general requires a description in terms of an open covering, and can be regarded as a simple example of computational descent theory. We also demonstrate a constructive version of a lemma of Hadian used in the computation of the Hodge filtration on $\mathscr{U}$ over affine elliptic and odd hyperelliptic curves. We use these algorithms to present some new examples describing the co-ordinates of some of these period maps. This description will be given in terms iterated $p$-adic Coleman integrals. We also consider the computation of the co-ordinates if we replace the rational basepoint with a tangential basepoint, and present some new examples here as well.
\end{abstract}

Keywords Elliptic curves - Hyperelliptic curves - de Rham fundamental group · Chabauty-Kim method · Unipotent Albanese map

Mathematics Subject Classification $11 \mathrm{GO} \cdot 11 \mathrm{G} 30 \cdot 11 \mathrm{~S} 80 \cdot 11 \mathrm{Y} 40$

\section{Résumé}

Nous étudions l'application unipotente d'Albanese qui apparait dans la méthode non- abélienne de Chabauty et Kim. En particulier, nous explorons le calcul explicite de l'application de de Rham $p$-adique de période $j^{d r}$ sur les courbes elliptiques et hyperelliptiques sur les corps numeriques via leurs connexions uniptotentes et universelles, $\mathscr{U}$. Sont inclus de nombreaux algorithmes qui font partie du calcul des versions de niveaux finis $j_{n}^{d r}$ de l'application unipotente d'Albanese. Le calcul de l'extension logarithmique de $\mathscr{U}$ nécessite une description par une couverture ouverte et peut être considérée comme un exemple de la théorie de la descendance computationelle. On montre aussi une version constructive d'un lemme d'Hadian utilisé dans le calcul de la filtration d'Hodge de $\mathscr{U}$, sur des courbes elliptiques

Jamie Beacom

beacom@maths.ox.ac.uk

1 Mathematical Institute, University of Oxford, Andrew Wiles Building, Radcliffe Observatory Quarter, Woodstock Road, Oxford OX2 6GG, UK 
affines ou hyperelliptiques affines impaires. Nous utilisons ces algorithmes pour présenter des nouveaux exemples décrivant les coordonnées de certaines de ces applications de périodes. La description sera donnée en terme des intégrales p-adiques itérées de Coleman. Nous considérons aussi le calcul des coordonnées quand le point de base rationnel est remplacé par un point de base tangentiel et présentons encore de nouveaux exemples dans ce cas.

\section{Contents}

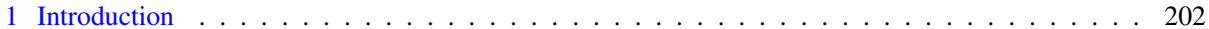

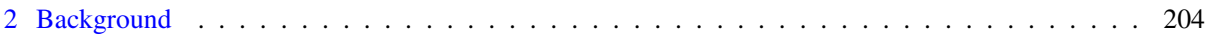

3 Logarithmic extensions of unipotent connections . . . . . . . . . . . . . . . . . . . 211

4 The Hodge filtration on $\mathscr{U} \ldots \ldots \ldots$. . . . . . . . . . . . . . . . . . . . . . . 227

5 Computing the de Rham period maps . . . . . . . . . . . . . . . . . . . . . . . 245

6 De Rham period maps with tangential basepoints . . . . . . . . . . . . . . . . . . . . . 250

7 Index of notation . . . . . . . . . . . . . . . . . . . . . . . . . 257

References . . . . . . . . . . . . . . . . . . . . . . . . 258

\section{Introduction}

This paper will examine some explicit aspects of a method introduced by Minhyong Kim in [23]. This method, known as the "Chabauty-Kim method" or "non-abelian Chabauty" is an analogue to and extension of an earlier method developed by Chabauty in [13] and made more explicit by Coleman in [16].

Suppose $X$ is a curve over a number field $K$. Let $v$ be a non-archimedean place of $K$, and $K_{v}$ its completion at $v$. The main restriction in the method of Chabauty-Coleman is the rank-genus condition, which requires our curve to have genus strictly greater than the rank of its Jacobian. Kim in [23] provides a possible way around this by replacing the Jacobian with a certain quotient of the de Rham fundamental group $U^{d r}$ of $X$, relative to some fixed basepoint $b \in X(K)$. The quotient in question comes from the Hodge filtration $F^{\bullet}$ on $U^{d r}$ defined in [26]. In [23] Kim shows that the quotient group $F^{0} U^{d r} \backslash U^{d r}$ naturally classifies the de Rham path spaces. This property of the quotient group allows us to define a higher de Rham unipotent Albanese map $j^{d r}$. Taking quotients of $U^{d r}$ by its lower central series yields a chain of sub-quotients $U_{n}^{d r}$, each of which also carries a Hodge filtration. Composition of the map $j^{d r}$ with the natural projection $U^{d r} \rightarrow U_{n}^{d r}$ give us a family of maps $j_{n}^{d r}: X\left(K_{v}\right) \rightarrow F^{0} U_{n}^{d r} \backslash U_{n}^{d r}$ which are compatible in the following sense:



The maps $j_{n}^{d r}$ have a Zariski dense image in the quotient $F^{0} U_{n}^{d r} \backslash U_{n}^{d r}$. It is this property which allows us to carry out a Chabauty type argument. Kim constructs a variety over $\mathbb{Q}_{p}$, the Selmer variety, with suitable finite level versions denoted here by $\mathrm{Sel}_{n}^{\text {glob }}$, along with finite 
level étale unipotent Albanese maps $j_{n}^{\text {ét }}: X(K) \rightarrow S e l_{n}^{\text {glob }}$. There is also an algebraic map $\log _{v, n}: S e l_{n}^{g l o b} \rightarrow F^{0} U_{n}^{d r} \backslash U_{n}^{d r}\left(K_{v}\right)$ such that the the image of $X(K)$ in $F^{0} U_{n}^{d r} \backslash U_{n}^{d r}\left(K_{v}\right)$ will be contained in the image of $S e l_{n}^{g l o b}$. The connection to Chabauty then is that if the following dimension hypothesis holds

$$
\operatorname{dim}\left(\operatorname{Sel} l_{n}^{g l o b}\right)<\operatorname{dim}\left(F^{0} U_{n}^{d r} \backslash U_{n}^{d r}\right)
$$

the algebraicity of $\log _{v, n}$ implies the existence of an algebraic function on $X\left(K_{v}\right)$ such that $X(K)$ is contained in its zero locus. The density of the image of $X\left(K_{v}\right)$ under $j_{n}^{d r}$ in $F^{0} U_{n}^{d r} \backslash U_{n}^{d r}$ implies that this algebraic function is non-zero on $X\left(K_{v}\right)$, and hence its zero locus is finite by the $p$-adic Weierstrass Preparation Theorem. The finiteness of $X(K)$ then follows.

One need not only consider $K$-rational points in the above. Let $R$ be the ring of integers of $K_{v}$ and let $S$ be some finite set of places of $K$ (which is usually taken to contain the places above primes of bad reduction for $X$ ). Then we can also try to use the machinery above to prove finiteness results for $\mathscr{X}\left(R_{S}\right)$, where $\mathscr{X}$ is some smooth model of $X$ over $R$ and $R_{S}$ are the $S$-integers of $K$.

There are a growing number of instances where the dimension hypothesis has been verified, yielding Diophantine applications. In a roughly chronological order, on the thrice punctured projective line $\mathbb{P}_{\mathbb{Q}}^{1}-\{0,1, \infty\}$ [22] it has been verified for the $\mathbb{Z}_{S}$-points and for the $S$ integers of a totally real field [21]; if $X$ is a once punctured elliptic curve with CM over $\mathbb{Q}$ then $n \geq \# S+r+1$ is sufficient to show the finiteness of $X\left(\mathbb{Z}_{S}\right)$ for some $r$ depending on $E$ [25] and if all the Tamagawa numbers are 1 and $X$ has rank 1 then $n=2$ is sufficient [24]; if $X$ is a complete hyperbolic curve with CM Jacobian [15]; and if $X$ is a complete hyperbolic curve and a solvable cover of $\mathbb{P}_{\mathbb{Q}}^{1}$ (and hence any smooth superelliptic curve over $\mathbb{Q}$ with genus at least 2 [20]. In [4,8] Balakrishnan and Dogra have made an explicit application of non-abelian Chabauty when $n=2$ - what they refer to as 'Quadratic Chabauty' - to $p$-adic heights on elliptic and hyperelliptic curves. They extended their methods in [7] to propose an effective Chabauty-Kim theorem which provides bounds of the type produced by Coleman under certain hypothesis even when $r=g$. Recently with Müller, Tuitman and Vonk they demonstrated an application of the Chabauty-Kim method in [9] to a non-hyperelliptic curve and used the method to complete the classification of non-CM elliptic curves over $\mathbb{Q}$ with split Cartan level structure. Also of interest is the fact that a number of important conjecturesBloch-Kato, Fontaine-Mazur and Jansenn-each imply the existence of an $n \gg 0$ such that the dimension hypothesis is satisfied yielding an effective form of Falting's Theorem.

The algebraic function whose existence is implied by the dimension hypothesis will be described locally as a $p$-adic analytic power series, and in fact will be defined by $p$-adic iterated integrals. These $p$-adic iterated integrals will come from the parallel transport associated to the $n$-th finite level quotient of the universal unipotent connection $\mathscr{U}$ associated to $X$.

The universal unipotent connection $\mathscr{U}$ is a universal object among pointed unipotent connections on $X$ and is in fact a pro-unipotent connection with finite level quotients $\mathscr{U}_{n}$. It transpires that the dual of $\mathscr{U}$ is the co-ordinate ring of the canonical $U^{d r}$ torsor $P^{d r}$. As outlined in [23] the universal connection comes with an associated Hodge filtration inducing the Hodge filtration on $U^{d r}$. As shall be shown in Sect.2, in order to determine the coordinates of $j_{n}^{d r}(x)$ it will be necessary to determine this Hodge filtration. Lemma 3.6 in [21] will be used to demonstrate how this Hodge filtration may be computed when $X$ is an affine elliptic or hyperelliptic curve. The practical computation of the Hodge filtration is motivated by the approach of Dogra in [19] and Balakrishnan and Dogra in [4]. 
As part of this computation the logarithmic extension $\overline{\mathscr{U}}$ of the universal connection $\mathscr{U}$ on $X$ will need to be computed. In Sect. 3 a general algorithm for computation of $\overline{\mathscr{U}}$ is outlined (Algorithm 1), which is an example of computational descent theory. That is, the logarithmic extension of the universal connection on the complement $X=C-D$ of a complete curve $C$ is computed as a collection of logarithmic connections on an open cover $\left(U_{i}\right)$ of $X$ together with isomorphism satisfying certain descent conditions. This will be necessary because unlike the case of $\mathbb{P}^{1} \backslash\{0,1, \infty\}$, for curves of positive genus the extension $\overline{\mathscr{U}}$ will in general have non-trivial bundle, or it may not be possible to express the connection on just one affine piece as having logarithmic poles at all the missing points. This is what necessitates taking the approach outlined above. The existence of suitable logarithmic extensions of the $\mathscr{U}_{n}$ compatible with projection is proven (Theorem 3). Conditions are imposed on the extensions, and using these two algorithms are provided, Algorithms 2 and 3, allowing for the iterative computation of $\overline{\mathscr{U}}_{n}$ for elliptic and odd hyperelliptic curves respectively. We then demonstrate how the computation of the Hodge filtration is contained in the computation of these extensions. In Theorem 4 we present a constructive version of Lemma 3.6 in [21] when $X$ is an elliptic curve or an odd hyperelliptic curve. From this we develop Algorithm 5 for the explicit computation of the Hodge filtration on the $\mathscr{U}_{n}$ in this case.

In Sect. 5 we apply the previous algorithms to the computation of $j_{n}^{d r}$ for several new $n$. Previously, $j_{n}^{d r}$ has been determined for elliptic curves only when $n=1,2$ and for hyperelliptic curves it has only been computed for $n=1,2$ in specific cases. We compute the co-ordinates of $j_{3}^{d r}$ (Proposition 12) and $j_{4}^{d r}$ (Proposition 13) for elliptic curves, and $j_{2}^{d r}$ (Proposition 14) for general odd hyperelliptic curves. In Sect. 6 we consider the scenario where our basepoint is tangential: this is useful in those cases where a rational basepoint is lacking, and to provide a greater wealth of examples. We provide new explicit descriptions of the coordinates of the maps $j_{2}^{d r}$ (Proposition 15) and $j_{3}^{d r}$ (Proposition 16) for elliptic curves with a tangential basepoint at infinity, and $j_{2}^{d r}$ (Proposition 17) for odd hyperelliptic curves with a tangential basepoint at infinity.

Although we concentrate on elliptic and odd degree hyperelliptic curves in this paper it should be possible to generalise much of the material to more general classes of curves. Affine elliptic curves and odd degree hyperelliptic curves, having one point at infinity removed from the complete curves, represented a relatively simply yet broad class of examples to consider. It should be simple to translate the results of Sect. 3 for more general curves. However, the conditions in Theorem 4 and the proof of said theorem are already very complicated and this is where I would expect to find some difficulty in generalising these results for more general curves.

Throughout this paper notation is introduced to describe the various gauge transformations and Hodge filtrations explicitly. At the recommendation of the reviewer, we have included an index of notation at the end of the paper in Section 7 which it is hoped will add clarity and aid referencing when using this paper.

\section{Background}

\subsection{The universal unipotent connection}

Here we introduce unipotent connections and background material on the unipotent Albanese map. Throughout both sections [23] is used as a primary reference for definitions and results.

Let $K$ be a field of characteristic 0 , let $X$ be a $K$-scheme, and suppose we have a fixed basepoint $b \in X(K)$. Let $\mathscr{V}$ be a vector bundle on $X$. 
Definition 1 A connection on $\mathscr{V}$ is a $K$-linear morphism of sheaves $\nabla$ such that

$$
\nabla: \mathscr{V} \rightarrow \mathscr{V} \otimes \Omega_{X / K}^{1}
$$

satisfying the Leibniz condition: for $U \subset X$ open, $\nabla(f s)=s \otimes d f+f \nabla(s)$ where $f \in \mathscr{O}_{X}(U), s \in \mathscr{V}(U)$. Here $\Omega_{X / K}^{1}$ is the sheaf of 1-forms on $X / K$.

Remark 1 We will often refer to a vector bundle $\mathscr{V}$ with connection $\nabla$ simply as a connection and write such objects either as $(\mathscr{V}, \nabla)$ or simply as $\mathscr{V}$.

Remark 2 We may extend $\nabla$ to a covariant derivative $\nabla^{1}: \mathscr{V} \otimes \Omega_{X / K}^{1} \rightarrow \mathscr{V} \otimes \Omega_{X / K}^{2}$ as follows: for $U \subset X$ open define $\nabla^{1}(s \otimes \omega):=s \otimes d \omega-\nabla(s) \wedge \omega$ for $s \in \mathscr{V}(U), \omega \in \Omega_{X / K}^{1}(U)$

Definition 2 We say that $\mathscr{V}$ is a flat or integrable connection if the induced morphism

$$
\nabla^{1} \circ \nabla: \mathscr{V} \rightarrow \mathscr{V} \otimes \Omega_{X / K}^{2}
$$

is the zero map. Note that if $X$ is a curve, then any connection $\mathscr{V}$ is automatically flat.

Given a connection $(\mathscr{V}, \nabla)$ with $\mathscr{V}$ of rank $n$, there is a matrix $\Omega \in \mathfrak{g l}_{n} \otimes \Omega_{X / K}^{1}$ called the connection matrix which determines $\nabla$ : suppose that we have a local basis $e_{i}: \mathscr{O}_{X} \hookrightarrow \mathscr{V}(1 \leq$ $i \leq n)$. Let $U \subset X$ be some trivialising neighbourhood in $X$. Then $\nabla\left(e_{i}\right) \in \mathscr{V} \otimes \Omega_{X / K}^{1}(U)$, and so there are $\omega_{i j} \in \Omega_{X / K}^{1}(U)$ such that

$$
\nabla\left(e_{i}\right)=\sum_{j} e_{j} \otimes \omega_{i j}
$$

We let $\Omega:=\left(\omega_{i j}\right)$. We may show that in matrix notation $\nabla(e \cdot f)=e \cdot(d f+\Omega \cdot f)$, and so $\nabla$ acts locally as $d+\Omega$.

Remark 3 A connection $(\mathscr{V}, \nabla=d+\Omega)$ is flat if and only if

$$
d \Omega+\Omega \wedge \Omega=0
$$

Definition 3 A morphism of connections $(\mathscr{V}, \nabla) \rightarrow\left(\mathscr{W}, \nabla^{\prime}\right)$ is a morphism $f: \mathscr{V} \rightarrow \mathscr{W}$ of sheaves preserving the connection.

Definition 4 A connection $\mathscr{V}$ is unipotent with index of unipotency less than or equal to $n$ if there is a decreasing sequence of sub-connections

$$
\mathscr{V}=\mathscr{V}_{n} \supset \mathscr{V}_{n-1} \supset \ldots \supset \mathscr{V}_{1} \supset \mathscr{V}_{0}
$$

such that the quotients $\mathscr{N}_{i+1} / \mathscr{V}_{i}$ are isomorphic to a direct sum of copies of $\left(\mathscr{O}_{X}, d\right)$ i.e. they are trivial.

We obtain the following category on $X$ :

Definition 5 Let $\operatorname{Un}_{n}(X)$ be defined to be category whose objects are unipotent vector bundles on $X$ with flat connection having index of unipotency less than or equal to $n$ with morphisms being morphisms of connections. Define $\mathrm{Un}(X)$ to be $\cup_{n \geq 1} \mathrm{Un}_{n}(X)$

Given some $b \in X(K)$ we can define a functor $e_{b}: \operatorname{Un}(X) \rightarrow \operatorname{Vect}_{K}$ from $\operatorname{Un}(X)$ to the category of vector spaces over $K$, sending $\mathscr{V} \mapsto \mathscr{V}_{b}:=b^{*} \mathscr{V}$. We may show that $e_{b}$ is a fibre functor $\left(\mathrm{Un}(X), e_{b}\right)$ is a neutral Tannakian category. In [1] the authors make the following definition: 
Definition 6 Given a neutral Tannakian category $(\mathscr{C}, \omega)$ over a field $k$, define the pointed category $\mathscr{C}^{*}$ to be the category whose objects are pairs $(V, v)$, where $V$ is an object of $\mathscr{C}$ and $v \in \omega(V)$ and morphisms $f:(V, v) \rightarrow(W, w)$ being morphisms $f: V \rightarrow W$ in $\mathscr{C}$ such that $\omega(f)(v)=w$.

With this in mind we may define the universal connection as a universal projective system of connections:

Definition 7 A projective system of objects $\left\{\left(\mathscr{U}_{n}, u_{n}\right)\right\}_{n \geq 0}$ in $\operatorname{Un}(X)^{*}$ with $\mathscr{U}_{n}$ having index of unipotency $\leq n$ for all $n \geq 0$ is called universal if for every $(\mathscr{V}, v)$ in $\operatorname{Un}(X)$ with index of unipotency $\leq n$ there is a unique morphism in $\operatorname{Un}(X)^{*} \phi$ such that

$$
\phi:\left(\mathscr{U}_{n}, u_{n}\right) \rightarrow(\mathscr{V}, v) .
$$

That is, there is a morphism $\phi: \mathscr{U}_{n} \rightarrow \mathscr{V}$ of connections such that

$$
\phi_{b}: u_{n}\left(\in b^{*} \mathscr{U}_{n}\right) \mapsto v\left(\in b^{*} \mathscr{V}\right) .
$$

It is shown in [27, I, Chapter 2] that such a universal projective system $\left\{\left(\mathscr{U}_{n}, u_{n}\right)\right\}_{n \geq 0}$ exists in $\operatorname{Un}(X)^{*}$. There it is the object referred to as the "generic pro-sheaf", $\mathscr{G}_{b, d r}$. Another construction is contained in Theorem A in [28]. A nice reference for the construction on a general scheme $X$ over a field $K$ is contained in [23, §1] where we see that we can take $\left(\mathscr{U}_{0}, u_{0}\right)=\left(\mathscr{O}_{X}, 1\right)$ and $u_{n}$ to be the point $1 \in b^{*} \mathscr{U}_{n}$ for all $n$.

Definition 8 Let $\left\{\mathscr{U}_{n}, 1\right\}_{n \geq 0}$ be a universal projective system in $\operatorname{Un}(X)^{*}$. Then we call this projective system the universal unipotent connection $(\mathscr{U}, u)$ on $X$.

Remark 4 By abuse of notation we will often also refer to the projective limit $\lim _{\leftarrow} \mathscr{U}_{n}$ as the universal connection $\mathscr{U}$. This is a pro-unipotent connection but as it does not have a finite index of unipotency it is not an object of $\operatorname{Un}(X)$. However, it will be useful to consider this pro-object in the next section where it's relationship to the de Rham fundamental group will be explored.

Remark 5 We will throughout this paper refer to the universal unipotent connection on $X$ simply as the universal connection on $X$.

When $X$ is an affine curve we have an explicit description of the universal unipotent pointed connection:

Definition 9 Let $C$ be a smooth projective curve of genus $g$ over a field $K$ of characteristic 0 . Let $D$ be a non-empty divisor of size $r$ and let $X:=C-D$. Let $\alpha_{0}, \ldots, \alpha_{2 g+r-2}$ be 1forms on $X$ such that their cohomology classes are a $K$-basis of $H_{d r}^{1}(X / K)$. We will assume that this basis is chosen so that the cohomology classes of $\alpha_{0}, \ldots, \alpha_{g-1}$ form a $K$-basis of $H^{0}\left(C, \Omega_{X / K}^{1}\right)$. Let $V_{d r}:=H_{d r}^{1}(X / K)^{\vee}$ with basis elements $A_{i}$ dual to $\alpha_{i}$. Let $R$ be the tensor algebra of $V_{d r}$ i.e.

$$
R:=\bigoplus_{k \geq 0} V_{d r}^{\otimes k}
$$

Write the basis element $A_{i_{1}} \otimes A_{i_{2}} \otimes \cdots \otimes A_{i_{k}}$ as the word $A_{i_{1}} A_{i_{2}} \ldots A_{i_{k}}$. Let $I$ be the two-sided ideal generated by $A_{0}, \ldots, A_{2 g+r-2}$ and define $R_{n}$ to be the quotient

$$
R_{n}:=R / I^{n+1}
$$


of $R$ by words of length $\geq n+1$. Then define

$$
\mathscr{U}_{n}:=R_{n} \otimes \mathscr{O}_{X}
$$

and let $\nabla_{n}$ be the connection such that

$$
f \in R_{n} \mapsto-\sum_{i} A_{i} f \otimes \alpha_{i} .
$$

For convenience we will often write $\nabla$ instead of $\nabla_{n}$.

Theorem 1 ([23], Lemma 3) Let X, $\mathscr{U}_{n}$ be as in Definition 9. For every $(\mathscr{V}, v)$ a pointed connection with index of unipotency $\leq n$ there is a unique map $\left(\mathscr{U}_{n}, 1\right) \rightarrow(\mathscr{V}, v)$.

We will need to consider filtrations on connections throughout this paper, and so we make the following definition:

Definition 10 By a filtered connection $\mathscr{V}:=\left(\mathscr{V}, \nabla, F^{\bullet}\right)$ on $X$ we mean a vector bundle $\mathscr{V}$ with a connection $\nabla$ which is equipped with a decreasing filtration by sub-bundles

$$
\mathscr{V}=F^{m} \mathscr{V} \subset F^{m+1} \mathscr{V} \subset \cdots \subset F^{n} \mathscr{V}=0
$$

for some $m<n \in \mathbb{Z}$ satisfying the Griffiths' transversality property:

$$
\nabla\left(F^{i} \mathscr{V}\right) \subset F^{i-1} \mathscr{V} \otimes \Omega_{X / K}^{1}
$$

for all $i$.

\subsection{The de Rham unipotent Albanese map}

From now on, we will assume the following: let $C$ be a smooth curve over a number field $K$ and $D$ a non-empty divisor defined over $K$. Then define $X:=C-D$ and let $b \in X(K)$ be a rational basepoint. Let $v$ be a non-Archimedean valuation on $K$ lying above a rational prime $p$ of good reduction for $X$ and take $K_{v}$ to be the completion of $K$ with respect to $v$. Let $R_{v}$ be the ring of integers of $K_{v}$ and $k$ its residue field. Finally, let $X_{v}:=X \otimes K_{v}$ denote the basechange of $X$.

Recall from Definition 5 that $\operatorname{Un}\left(X_{v}\right)$ is defined to be the category of unipotent connections on $X_{v}$ with finite unipotency index. Note we have dropped the requirement that the connections are flat here since $X_{v}$ is a curve and, therefore, $\Omega_{X_{v} / K_{v}}^{2}=0$. Given $b \in X(K)$ then the functor

$$
e_{b}: \operatorname{Un}\left(X_{v}\right) \mapsto \operatorname{Vect}_{K_{v}} ; \mathscr{V} \mapsto b^{*} \mathscr{V}
$$

is a fibre functor and $\left(\mathrm{Un}\left(X_{v}\right), e_{b}\right)$ is a neutral Tannakian category. We denote by $\left\langle\operatorname{Un}_{n}\left(X_{v}\right)\right\rangle$ the Tannakian sub-category of $\operatorname{Un}\left(X_{v}\right)$ generated by $\operatorname{Un}_{n}\left(X_{v}\right)$ and we let $\left\langle e_{b}^{n}\right\rangle$ denote the restriction of $e_{b}$ to this sub-category. Using Tannaka duality we make the following definition:

Definition 11 The de Rham fundamental group $U^{d r}$ of $X_{v}$ with basepoint $b$ is that group scheme associated to the Tannakian category $\left(\operatorname{Un}\left(X_{v}\right), e_{b}\right)$ representing $\operatorname{Aut}^{\otimes}\left(e_{b}\right)$. There is also a group scheme $U_{n}^{d r}$ associated to the Tannakian category $\left(\left\langle\operatorname{Un}_{n}\left(X_{v}\right)\right\rangle,\left\langle e_{b}^{n}\right\rangle\right)$ and representing Aut ${ }^{\otimes}\left(\left\langle e_{b}^{n}\right\rangle\right)$. For $x \in X\left(K_{v}\right)$ the de Rham path torsor $P^{d r}(x)$ is the right $U^{d r}$. torsor representing $\operatorname{Isom}^{\otimes}\left(e_{b}, e_{x}\right)$ and $P_{n}^{d r}(x)$ similarly is the right $U_{n}^{d r}$-torsor representing $\operatorname{Isom}^{\otimes}\left(\left\langle e_{b}^{n}\right\rangle,\left\langle e_{x}^{n}\right\rangle\right)$. 
Remark 6 The group scheme $U_{n}^{d r}$ is a quotient of $U^{d r}$ in the following sense: let $G$ be a group scheme and define $Z^{1} G:=G$ and for $n \geq 1$ let $Z^{n+1} G:=\left[G, Z^{n} G\right]$. Then we have $U_{n}^{d r}=U^{d r} / Z^{n} U^{d r}$.

Remark 7 The $P^{d r}(x)$ fit together to form the canonical torsor $P^{d r} \rightarrow X$ which is a right torsor for $X \times{ }_{K_{v}} U^{d r}$ with fibre over $x \in X\left(K_{v}\right)$ being $P^{d r}(x)$. Similarly there is a canonical torsor $P_{n}^{d r}$ for $U_{n}^{d r}$.

We now elucidate the relationship between the universal connections of the previous section and the de Rham fundamental group through the following lemmas and propositions. We omit the proofs, but these can be found in [23].

Lemma 1 There are functorial isomorphisms

$$
x^{*} \mathscr{U}_{n} \cong \operatorname{Hom}\left(e_{b}^{n}, e_{x}^{n}\right) ; x^{*} \mathscr{U} \cong \operatorname{Hom}\left(e_{b}, e_{x}\right) .
$$

Lemma 2 Let $\mathscr{U}_{n}$ be as in Definition 9. Then there is a unique morphism of connections $\Delta: \mathscr{U}_{n+m} \mapsto \mathscr{U}_{n} \otimes \mathscr{U}_{m}$ such that $\Delta(1)=1 \otimes 1$ and $\Delta\left(A_{i}\right)=A_{i} \otimes 1+1 \otimes A_{i}$.

Remark 8 This morphism of connections $\Delta$ extends to $\mathscr{U}$ by taking the limits over all $m$ and $n$. This in turn makes $\mathscr{U}$ into a sheaf of co-commutative co-algebras. Recall from the previous lemma that there is a functorial isomorphism $\lim _{\leftarrow} R_{n}=x^{*} \mathscr{U} \cong \operatorname{Hom}\left(e_{b}, e_{x}\right)$. Following [23, Section 1] note that $b^{*} \mathscr{U}$ is the universal enveloping algebra of $\operatorname{Lie}\left(U^{d r}\right)$. This then is a co-commutative Hopf algebra and the co-product will be that induced by the map $\Delta$ of the previous lemma.

Definition 12 The group-like elements of the Hopf algebra $b^{*} \mathscr{U}$ are the $g \in b^{*} \mathscr{U}$ such that $\Delta(g)=g \otimes g$. The primitive elements are the $h \in b^{*} \mathscr{U}$ such that $\Delta(h)=h \otimes 1+1 \otimes h$.

Using the functoriality of the isomorphism $x^{*} \mathscr{U} \cong \operatorname{Hom}\left(e_{b}, e_{x}\right)$ together with it's explicit description as in [23] we deduce that $f \in x^{*} \mathscr{U}$ is group-like if and only if it belongs to $U^{d r}$. From this we may deduce the following result.

Proposition 1 The coordinate ring $\mathscr{P}^{d r}$ of $P^{d r}$ is the dual sheaf $\mathscr{P}=\mathscr{U}^{\vee}$.

Remark 9 Following this, $U^{d r}$ is then identified with the group-like elements of $b^{*} \mathscr{U}$ and $\operatorname{Lie}\left(U^{d r}\right)$ is identified with the primitive elements. The exponential map

$$
\exp : g \mapsto \sum_{n=0}^{\infty} \frac{g^{n}}{n !}
$$

converges on the image of $\operatorname{Lie}\left(U^{d r}\right)$ in each $R_{n}=b^{*} \mathscr{U}_{n}$. It is simple to see that when $g$ is primitive and $\exp (g)$ converges then $\exp (g)$ is group-like. Similarly, there is a logarithm map

$$
\log : g \mapsto \sum_{n=1}^{\infty} \frac{(1-g)^{n}}{n}
$$

which, when it converges, is inverse to the exponential map. Thus, for any $K_{v}$-algebra $A$ we have an isomorphism

$$
\exp : \operatorname{Lie}\left(U^{d r}\right) \otimes A \cong U^{d r}(A) .
$$


Using Proposition 1 we can define a filtration on $P^{d r}$ which turns out to be a filtration by lengths of iterated integrals.

Definition 13 Let $\mathscr{P}_{n}^{d r}:=\mathscr{U}_{n}^{\vee}$. The Eilenberg-Maclane filtration on $P^{d r}=\operatorname{Spec}(\mathscr{P})$ is defined by

$$
\mathscr{O}_{X} \subset \mathscr{P}_{1}^{d r} \subset \mathscr{P}_{2}^{d r} \subset \cdots \subset \mathscr{P}^{d r}=\mathscr{U}^{\vee}
$$

The projection $\mathscr{U} \rightarrow \mathscr{U}_{n}$ corresponds then to the projection $P^{d r} \rightarrow P_{n}^{d r}$. One may then consider the co-product $\Delta$ on the fibres $x^{*} \mathscr{U}_{n}$ and identify the group-like elements with those of $P_{n}^{d r}$. In [28, Theorem E] Wojtkowiak demonstrates that $\mathscr{P}^{d r}$ possess a Hodge filtration $F^{\bullet}$ :

$$
\mathscr{P}^{d r}=F^{0} \mathscr{P}^{d r} \supset \cdots \supset F^{i} \mathscr{P}^{d r} \supset \cdots
$$

by sub $\mathscr{O}_{X}$-modules where the $F^{i} \mathscr{P}^{d r}$ are all ideals. The filtration $F^{\bullet} \mathscr{P} d r$ in turn induces a filtration on $P^{d r}$ :

Definition 14 The Hodge filtration is such that $F^{i} P^{d r}$ has defining ideal $F^{-i+1} \mathscr{P} d r$.

In loc.cit. it is shown that with the induced filtration $F^{\bullet} U^{d r}$ on $U^{d r}$ the space $F^{0} P^{d r}$ becomes an $F^{0} U^{d r}$ torsor and is hence trivialised over an $K_{v}$-algebra $Z$ as $F^{0} U^{d r}$ is unipotent.

Now let $Y$ be the reduction of $X_{v}$ over $k$. Let $\operatorname{Un}(Y)$ be the category of overconvergent unipotent isocrystals on $Y$. If we basechange to $K_{v}$, this will be identified with unipotent connections convergent on every residue disk on $X$, and overconvergent near points of $D_{v}=$ $D \otimes K_{v}$. For $c \in Y(k)$, let $] c[$ denote the residue disk of $c$. Then there is a fibre functor $e_{c}:(\mathscr{V}, \nabla) \mapsto \mathscr{V}(] c[)^{\nabla=0}$ which takes the horizontal sections of $\mathscr{V}$ on the residue disk of $c$. Tannaka duality then gives us a crystalline fundamental group $U^{c r}$, and a right-torsor of crystalline paths $P^{c r}(y)$ for $y \in Y(k)$. We similarly obtain $U_{n}^{c r}, P_{n}^{c r}(y)$ with overconvergent isocrystals of unipotency index less than or equal to $n$.

The $q=|k|$-power map on $\mathscr{O}_{Y}$ induces a Frobenius automorphism $\phi: P_{n}^{c r}(y) \simeq P_{n}^{c r}(y)$. By the comparison theorem of Chiarellotto ([14]) between de Rham and crystalline fundamental groups we obtain a Frobenius automorphism $\phi$ on $P_{n}^{d r}(x)$. Besser shows that this Frobenius automorphism satisfies the following property:

Theorem 2 ([11], Theorem 3.1) The map $U_{n}^{d r} \rightarrow U_{n}^{d r}$ given by $g \mapsto \phi(g) g^{-1}$ is an isomorphism.

As a consequence of this Besser shows in [11, Corollary 3.2] that one may deduce that for any $x \in X_{v}\left(K_{v}\right)$ there is a unique Frobenius-invariant de Rham path $p_{n}^{c r}(x)$ from $b$ to $x$. Both existence and uniqueness follow in a fairly straightforward manner from the above theorem. We are now ready to define the de Rham period map. In [23] Kim defines the notion of an admissible $U^{d r}$-torsor.

Let $T=\operatorname{Spec}(\mathscr{T})$ be a right $U_{n}^{d r}$-torsor over a $K_{v}$-scheme $Z$. We say that a $U^{d r}$-torsor $T$ is admissible if it has an Eilenberg-Maclane filtration; a Hodge filtration such that $F^{0} T$ is trivialised over $Z$; it has a Frobenius morphsim of $Z$-schemes $\phi$ semilinear with respect to the $U^{d r}$-action and preserving the Eilenberg-Maclane filtration; $\phi$ has a unique invariant $Z$-point; and, there is a universal injectivity property on the filtrations.

Remark 10 Given our previous results observe then that $P^{d r}$ and $P_{n}^{d r}$ are admissible torsors for $U^{d r}$ and $U_{n}^{d r}$ respectively. 
Let $T$ be an admissible torsor over a $K_{v}$-algebra $L$. Then as it is a right torsor, there is a $u_{T} \in U_{n}^{d r}$ such that $p_{T}^{c r}=p_{T}^{H} u_{T}$. The point $u_{T}$ will be unique up to multiplication on the left by $F^{0} U_{n}^{d r}$, and so we have a $\left[u_{T}\right] \in F^{0} \mathscr{U}_{n}^{d r} \backslash U_{n}^{d r}$. There leads to the following bijective correspondence.

Proposition 2 (Kim, [23] Proposition 1) There is a natural bijection between $F^{0} U_{n}^{d r} \backslash U_{n}^{d r}$ (resp. $F^{0} U^{d r} \backslash U^{d r}$ ) and isomorphism classes of admissble $U_{n}^{d r}$-torsors (resp. admissible $U^{d r}$-torsors) given by the map

$$
T \mapsto\left[u_{T}\right] .
$$

We are now in a position to define the de Rham period map, which is the de Rham realisation of the unipotent Albanese map. Note here that we will write $\left[P^{d r}(x)\right]$ as the image of $P^{d r}(x)$ under the map from the preceding proposition rather than $\left[u_{P^{d r}(x)}\right]$.

Definition 15 The de Rham period maps $j^{d r}, j_{n}^{d r}$ are defined as follows:

$$
\begin{aligned}
j^{d r} & : X_{v}\left(K_{v}\right) \rightarrow F^{0} U^{d r} \backslash U^{d r} \\
& x \mapsto\left[P^{d r}(x)\right] \\
j_{n}^{d r}: & X_{v}\left(K_{v}\right) \rightarrow F^{0} U^{d r} \backslash U^{d r} \rightarrow F^{0} U_{n}^{d r} \backslash U_{n}^{d r} \\
& x \mapsto\left[P^{d r}(x)\right] \mapsto\left[P_{n}^{d r}(x)\right]
\end{aligned}
$$

In applications to Diophantine problems it is the finite level maps $j_{n}^{d r}$ that we are primarily interested in. Thus our aim should be to find explicit representatives for $\left[P_{n}^{d r}(x)\right]$ in $F^{0} U_{n}^{d r} \backslash U_{n}^{d r}$ for arbitrary $x \in X_{v}\left(K_{v}\right)$. To do this we need to find a Frobenius invariant $p_{n}^{c r}(x) \in P_{n}^{d r}(x)$, a trivialisation $p_{n}^{H}(x) \in F^{0} P_{n}^{d r}(x)$ and $u_{n}(x) \in U_{n}^{d r}$ such that $p_{n}^{c r}(x)=p_{n}^{H}(x) u_{n}(x)$. Then we can take $\left[P_{n}^{d r}(x)\right]=\left[u_{n}(x)\right]$. The element $p_{n}^{c r}(x)$ is computed as the parallel transport of $1 \in b^{*} \mathscr{U}_{n}$ to the fibre $x^{*} \mathscr{U}_{n}$.

Lemma 3 ([23], §1) The Frobenius invariant path $p_{n}^{c r}(x)$ in $P_{n}^{d r}(x)$ is given by

$$
p_{n}^{c r}(x)=1+\sum_{|w| \leq n} \int_{b}^{x} \alpha_{w} w
$$

where $\alpha_{w}=\alpha_{i_{1}} \alpha_{i_{2}} \cdots \alpha_{i_{s}}$ if $w=A_{i_{1}} A_{i_{2}} \cdots A_{i_{s}}$ and the sum is taken over all words in $A_{0}, \ldots, A_{2 g-r+2}$ of length at most $n$.

Remark 11 In the above lemma the iterated integrals appearing are iterated Coleman integrals defined by

$$
\int_{x}^{y} \omega_{1} \ldots \omega_{r}:=\int_{x}^{y} \omega_{1}\left(t_{1}\right) \int_{x}^{t_{1}} \omega_{2}\left(t_{2}\right) \ldots \int_{x}^{t_{r-1}} \omega_{r-1}\left(t_{r-1}\right) \int_{x}^{t_{r}} \omega_{r}\left(t_{r}\right) .
$$

Balakrishnan has developed algorithms for computing iterated Coleman integrals of this type on elliptic and hyperelliptic curves (see $[3,5]$ ) which have seen applications to Kim's non-abelian Chabauty.

Determining $p_{n}^{H}(x)$ will requires us to be able to compute the Hodge filtration on $P_{n}^{d r}$. The filtration $F^{\bullet} \mathscr{P} d r$ induces a filtration on the dual $\mathscr{U}$ and each quotient $\mathscr{U}_{n}$. This will give each $\mathscr{U}_{n}$ the structure of a filtered connection and by computing this filtration we may identify the filtration on $P^{d r}$ and $P_{n}^{d r}$. In what follows we spend some time showing how this may be explicitly calculated in the case of elliptic curves and odd hyperelliptic curves. 


\section{Logarithmic extensions of unipotent connections}

In the introduction we noted that in order to compute the Hodge filtration on the universal pointed connection of an affine curve we will need to make use of an approach due to Hadian in [21]. This will require us to compute a universal projective system of logarithmic connections on the compact curve extending the universal projective system of connections on the affine curve. This section is concerned with presenting a computational method to do this in the case that we have elliptic or odd hyperelliptic curves.

Let $C, D$ and $X$ be as in Definition 9 and let $\Omega_{C}^{1}(D)$ be the sheaf of logarithmic differentials on $C$ along $D$. This sheaf will consist of differentials on $C$ regular on $X$ and with at worst logarithmic poles along $D$.

Definition 16 A logarithmic connection on $C$ with logarithmic poles along $D$ is a vector bundle $\mathscr{V}$ equipped with a $K$-linear morphism of sheaves $\nabla$ such that

$$
\nabla: \mathscr{V} \rightarrow \mathscr{V} \otimes \Omega_{C}^{1}(D)
$$

satisfying the Leibniz condition as in Definition 1. A trivial logarithmic connection on $C$ along $D$ is a direct sum of copies of $\left(\mathscr{O}_{C}, d\right)$ where $\mathscr{O}_{C}$ is the structue sheaf of $C$ and $d$ is its exterior derivative. A morphism of logarithmic connections is defined analogously to morphisms of connections.

For any open $Y \subset C$ a logarithmic connection on $C$ with poles along $D$ is a vector bundle $\mathscr{W}$ equipped with a $K$-linear morphism of sheaves

$$
\nabla: \mathscr{V} \rightarrow \mathscr{V} \otimes \Omega_{Y}^{1}(D \cap Y)
$$

Definition 17 Let $\mathscr{V}$ be a connection on $X=C-D$. A logarithmic extension of $\mathscr{V}$ to $C$ along $D$ is a logarithmic connection $\overline{\mathscr{V}}$ on $C$ with logarithmic poles along $D$ such that $\left.\overline{\mathscr{V}}\right|_{X} \cong \mathscr{V}$.

That such extensions exist is a consequence of a theorem of Deligne ([17, Proposition 5.2]). In this section we provide some algorithms to compute such extensions when $C$ is either an elliptic or odd hyperelliptic curve and $D$ consists of the point at infinity.

In [21] Hadian defines unipotent logarithmic connections, which are iterated extensions of trivial logarithmic connections $\left(P \otimes_{K} \mathscr{O}_{C}, \mathrm{id}_{P} \otimes_{K} d\right)$. Here $P$ is a finite dimensional $K$-vector space and $d: \mathscr{O}_{C} \rightarrow \Omega_{C}^{1}(D)$ is the usual exterior derivative. The logarithmic extension $\overline{\mathscr{U}}$ of the universal connection $\mathscr{U}$ on the affine curve $X$ is then used as a model for $\mathscr{U}$. Lemma 3.6 in loc.cit. suggests a practical way to compute the Hodge filtration of $\mathscr{U}$ by making use of this logarithmic extension $\overline{\mathscr{U}}$ and we will employ this approach in Sect. 4. We now turn our attention to the computation of the logarithmic extension of $\mathscr{U}$.

\subsection{A general algorithm for logarithmic extensions}

Recall that $\mathscr{U}$ consists of a projective system of pointed unipotent connections $\left\{\left(\mathscr{U}_{n}, u_{n}\right)\right\}$. We compute the logarithmic extension of $\mathscr{U}$ as a projective system $\overline{\mathscr{U}}=\left\{\left(\overline{\mathscr{U}}_{n}, u_{n}\right)\right\}$ where each $\overline{\mathscr{U}}_{n}$ is a logarithmic extension of $\mathscr{U}_{n}$. We shall see later that this construction will ensure that the resulting projective system is a universal projective system among pointed unipotent logarithmic connections. In order to compute logarithmic extensions $\overline{\mathscr{U}}_{n}$ of the $\mathscr{U}_{n}$ we utilise a description in terms of an open covering of $C$. That is, an object is described on open subsets of some cover together with gluing morphisms (descent datum) which satisfy some cocycle condition. 
Definition 18 Descent for logarithmic connections on $C$ along $D$ is given by the following descent datum:

1. An open cover $\left(Y^{i}\right)_{i}$ of $C$

2. Logarithmic connections $\mathscr{V}_{i}=\left(\mathscr{O}_{Y^{i}}^{r}, \nabla_{i}\right)$ with poles along $Y^{i} \cap D$

3. Isomorphisms of logarithmic connections $G_{i j}:\left(\mathscr{O}_{Y^{i j}}^{r},\left.\nabla_{i}\right|^{Y_{i j}}\right) \stackrel{\sim}{\rightarrow}\left(\mathscr{O}_{Y^{i j}}^{r},\left.\nabla_{j}\right|_{Y^{i j}}\right)$ such that for all $i$ we have $\left.G_{i i}=\operatorname{id}_{\left(\mathscr{O}_{Y i}^{r}\right.}, \nabla_{i}\right)$ and such that for all $i, j, k$ the following cocycle condition is satisfied:

$$
\left(\left.G_{j k}\right|_{Y^{i j k}}\right) \circ\left(\left.G_{i j}\right|_{Y^{i j k}}\right)=\left(\left.G_{i k}\right|_{Y^{i j k}}\right)
$$

This descent datum will be written as $\left(\mathscr{V}_{i}, G_{i j}\right)$.

To construct the descent datum we will need the following lemma which follows from an easy computation.

Lemma 4 Given a (logarithmic) connection $\mathscr{V}$ on a curve $Z$, suppose that with respect to the local basis $\left(e_{i}\right)$ it has connection matrix $\Omega$. If $G$ is an automorphism of $\mathscr{V}$, the transformation

$$
\Omega \mapsto G^{-1} d G+G^{-1} \Omega G
$$

is called a gauge transformation of $\Omega$. This is the connection matrix of $\mathscr{V}$ with respect to the local basis $\left(G^{-1} e_{i}\right)$.

We now turn our attention towards computing the logarithmic extensions of the connections $\mathscr{U}_{n}$. We want an iterative algorithm by which we may compute the logarithmic extensions $\overline{\mathscr{U}}_{n}$ of the $\mathscr{U}_{n}$ successively. Observe that $\overline{\mathscr{U}}_{0}=\left(\mathscr{O}_{C}, d\right)$ is a logarithmic extension of $\mathscr{U}_{0}=\left(\mathscr{O}_{X}, d\right)$ and we take this as our base case. The construction of the extensions $\overline{\mathscr{U}}_{n}$ is based upon the following observation in [21]:

Proposition 3 ([21], Lemma 2.3 \& Proposition 2.6) For every $n \geq 0$ there exists an extension $\overline{\mathscr{U}}_{n+1}$ of $\overline{\mathscr{U}}_{n}$ by $\left(V_{d r}^{\otimes(n+1)} \otimes \mathscr{O}_{C}, d\right)$ such that $1 \in b^{*} \overline{\mathscr{U}}_{n+1}$ maps to $1 \in b^{*} \overline{\mathscr{U}}_{n}$ under projection. Let $\mathscr{V}$ be a unipotent logarithmic connection on $C$ with poles along $D$ of unipotency index $m$. Then for all $v \in b^{*} \mathscr{V}$ and $n \geq m$ there exists a unique morphism $\phi_{v}: \overline{\mathscr{U}}_{n} \rightarrow \mathscr{V}$ and $1\left(\in b^{*} \overline{\mathscr{U}}_{n}\right) \mapsto v \in\left(b^{*} \mathscr{V}\right)$.

In the parlance of Definition 7 we then say that $\left\{\left(\overline{\mathscr{U}}_{n}, 1\right)\right\}$ forms a universal projective system in the category of pointed unipotent logarithmic connections on $C$ with logarithmic poles along $D$ and we denote this projective system by $\overline{\mathscr{U}}$. By an abuse of notation we will also denote the universal pro-unipotent logarithmic connection $\lim _{\leftarrow} \overline{\mathscr{U}}_{n}$ by $\overline{\mathscr{U}}$ when the context is clear. In light of Proposition 3 we will construct finite level extensions $\overline{\mathscr{U}}_{n}$ as extensions fitting into an exact sequence

$$
0 \rightarrow V_{d r}^{\otimes(n+1)} \otimes \mathscr{O}_{C} \rightarrow \overline{\mathscr{U}}_{n+1} \rightarrow \overline{\mathscr{U}}_{n} \rightarrow 0
$$

of logarithmic connections. For each $n$ we shall, therefore, require a suitable projection map $\overline{\mathscr{U}}_{n} \rightarrow \overline{\mathscr{U}}_{n-1}$. To incorporate this into the construction we need to define we what we mean by morphisms of descent data. In the following definition we assume that both descent data are described over a single covering of $C$. 
Definition 19 A morphism of descent data $\left(\mathscr{V}_{i}, G_{i j}\right)$ and $\left(\mathscr{V}_{i}^{\prime}, G_{i j}^{\prime}\right)$ for a logarithmic connection on $C$ along $D$ is given by a family $\rho=\left(\rho_{i}\right)_{i}$ of morphisms of logarithmic connections $\rho_{i}: \mathscr{V}_{i} \rightarrow \mathscr{V}_{i}^{\prime}$ such that all of the diagrams

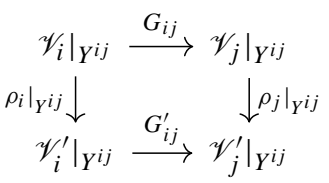

commute.

We compute gauge transformations $G$ which map the connection matrix of $\mathscr{U}_{n+1}$ over $X$ to a connection matrix with at worst logarithmic poles at the points of $D$. The gauge transformations will be the transition functions for the descent data. We then determining a suitable open cover $\left(Y_{n+1}^{i}\right)_{i}$ of $C$ such that these connection matrices define logarithmic connections $\mathscr{U}_{n+1}^{i}$ on each of the patches $Y_{i}^{n+1}$. Over the patch $Y_{i}^{n+1}$ we simply define the extension to have bundle $R_{n+1} \otimes \mathscr{O}_{Y_{n+1}^{i}}$. This is done subject to the condition that for each candidate open patch $Y_{n+1}^{i}$ we should have a commutative diagram

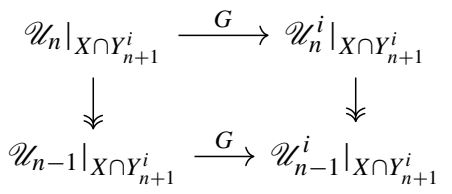

Then the logarithmic connections on the $Y_{n+1}^{i}$ with poles along together with the connection $\mathscr{U}_{n}$ on $X$ and the gauge transformations define descent datum for a logarithmic connection on $C$ with $\log$ poles along $D$. Note that it will be convenient for the computations that follow to describe the gauge transformations $G$ as elements of $K(C) \otimes_{K} \mathfrak{g l}_{N}$ for some $N$.

Algorithm 1 (Computing the logarithmic extension of the universal connection on $X=$ $C-D)$

\section{Input}

- A smooth projective curve $C$ over a field $K$ of characteristic 0 , non-empty divisor $D=$ $\left\{d_{1}, \ldots, d_{r}\right\}$ defined over $K, X=C-D$

- The universal connection $\mathscr{U}_{n}$ on $X=Y_{n}^{0}=Y_{n+1}^{0}$ with respect to a basis of $H_{d r}^{1}(X)$

- The logarithmic extension $\overline{\mathscr{U}}_{n}$ of $\mathscr{U}_{n}$ defined by the following descent datum:

1. Trivial logarithmic connections $\left(\mathscr{U}_{n}^{i}, d+C_{n}^{i}\right)$ over open subsets $Y_{n}^{i} \subset C$ where $d_{i} \in Y_{n}^{i}$ for $i \neq 0$ and $\left(Y_{n}^{i}\right)_{i}$ a cover of $C$

2. Gauge transformations $G_{n}^{i j}$ with:

- $G_{n}^{i j}: C_{n}^{i} \mapsto C_{n}^{j}$ on $Y_{n}^{i j}=Y_{n}^{j} \cap Y_{n}^{i}$ compatible with projections to level $n-1$ for all $i, j$

- $G_{n}^{i j}=\left(G_{n}^{j i}\right)^{-1}$ on $Y_{n}^{i j}$ for all $i, j$

- $G_{n}^{i i}=\operatorname{id}$ on $Y_{n}^{i i}$ for all $i$

- $G_{n}^{j k} \circ G_{n}^{i j}=G_{n}^{i k}$ on $Y_{n}^{i j k}=Y_{n}^{i} \cap Y_{n}^{j} \cap Y_{n}^{k}$ for all $i, j, k$ (cocycle condition) 


\section{Output}

- The logarithmic extension $\overline{\mathscr{U}}_{n+1}$ of $\mathscr{U}_{n+1}$ defined by the following descent datum:

1. Trivial logarithmic connections $\left(\mathscr{U}_{n+1}^{i}, d+C_{n+1}^{i}\right)$ over open subsets $Y_{n+1}^{i} \subset C$ with $d_{i} \in Y_{n+1}^{i}$ for $i \neq 0$ and $\left(Y_{n+1}^{i}\right)_{i}$ a cover of $C$

2. Gauge transformations $G_{n+1}^{i j}$ with:

- $G_{n+1}^{i j}: C_{n+1}^{i} \mapsto C_{n+1}^{j}$ on $Y_{n+1}^{i j}=Y_{n+1}^{j} \cap Y_{n+1}^{i}$ compatible with projections to level $n$ for all $i, j$

- $G_{n+1}^{i j}=\left(G_{n+1}^{j i}\right)^{-1}$ on $Y_{n+1}^{i j}$ for all $i, j$

- $G_{n+1}^{i i}=\mathrm{id}$ on $Y_{n+1}^{i i}$ for all $i$

- $G_{n+1}^{j k} \circ G_{n+1}^{i j}=G_{n+1}^{i k}$ on $Y_{n+1}^{i j k}=Y_{n+1}^{i} \cap Y_{n+1}^{j} \cap Y_{n+1}^{k}$ for all $i, j, k$ (cocycle condition)

\section{Algorithm}

(1) For $i \in\{1, \ldots, r\}$

(a) Compute a gauge transformation $G_{n+1}^{0 i}$ of $C_{n+1}$, the connection matrix of $\mathscr{U}_{n+1}$, such that

- $G_{n+1}^{0 i}$ is compatible with projection to level $n$

- the image $C_{n+1}^{i}$ of $C_{n+1}$ has at worst logarithmic poles at $d_{i}$

(2) For $i \in\{1, \ldots, r\}$

(a) Choose open $Y_{n+1}^{i} \subset C$ containing $d_{i}$ such that $C_{n+1}^{i}$ has no poles on $Y_{n+1}^{i}$ except possibly at $d_{i}$; together with $Y_{n+1}^{0}=X$ these cover $C$

(b) Let $\mathscr{U}_{n+1}^{i}=R_{n+1} \otimes \mathscr{O}_{Y_{n+1}^{i}}$

(c) Give $\mathscr{U}_{n+1}^{i}$ the logarithmic connection $d+C_{n+1}^{i}$.

(3) Define:

- $G_{n+1}^{j 0}:=\left(G_{n+1}^{0 j}\right)^{-1}$ for all $j$

- $G_{n+1}^{i i}:=$ id for all $i$

- $G_{n+1}^{i j}:=G_{n+1}^{0 j}\left(G_{n+1}^{0 i}\right)^{-1}$ for all $i, j$

(4) Glue the logarithmic connections $\mathscr{U}_{n+1}^{i}, \mathscr{U}_{n+1}$ together via the isomorphisms $G_{n+1}^{i j}$ to obtain a logarithmic connection $\overline{\mathscr{U}}_{n+1}$ with $\log$ poles along $D$.

Remark 12 It should be noted that the above algorithm relies on the ability to complete Step 1) a) for each $i \in\{1, \ldots, r\}$. The next two sections show that we can do this in the case that $C$ is an elliptic curve or an odd model of a hyperelliptic curve.

Remark 13 It may be the case that several of the opens $Y_{n+1}^{i}$ coincide i.e. that a single $Y_{n+1}^{i}$ may be chosen to contain several of the points of $C$ missing from $X$.

Remark 14 Although the above algorithm implies that the choice of open cover $\left(Y_{n}^{i}\right)_{i}$ at each level of iteration depends on $n$, the results in Sect.3.2 and 3.3 show that we can often eliminate this dependence on $n$ through some judicious choices for $G_{n}^{i j}$. It should be possible to replicate this in other more general examples of curves by choosing the entries of the $G_{n}^{0 i}$ to be polynomial in some $F$ with a single simple pole at the point $d_{i}$ where possible. 
Remark 15 As noted in the opening paragraph of this section the logarithmic connection $\overline{\mathscr{U}}$ constructed above is universal among pointed unipotent logarithmic connections on $C$ with poles along $D$. In the following sections we shall see that there is a certain amount of choice available when computing these extensions. These different choices give rise to isomorphic unipotent logarithmic connections each of which satisfies the universal property described in Definition 7.

Remark 16 As the universal connection $\tilde{\mathscr{U}}$ on $C$ is also a pointed logarithmic connection there is a morphism of pointed logarithmic connections $\Phi: \overline{\mathscr{U}} \rightarrow \widetilde{\mathscr{U}}$. We thus exhibit $\tilde{\mathscr{U}}$ as a maximal quotient of $\overline{\mathscr{U}}$ without poles.

\subsection{Logarithmic extensions on affine elliptic curves}

In what follows, we describe this process explicitly for an arbitrary elliptic curve. However, it should be noted that the results presented in this section should easily translate to any general smooth projective curve $C$ punctured at $r$ points. Replacing the dimensions of $H_{d r}^{1}(X / K)$ with a variable $s:=2 g+r-2$ the same results will still apply. However, where $r>1$ care must be taken to compute the logarithmic extension near each of the punctured points individually then glue the resulting logarithmic connections together. This would add another layer of notational complexity in what is admittedly an already notation heavy set of results, which is why we have elected here to stick to a simpler example.

Let $C$ be an elliptic curve over a field $K$ of characteristic 0 with $K$-rational point at infinity $\infty$. Let $X:=C-\{\infty\}$ be the punctured elliptic curve with model $y^{2}=f(x)$ where $f(x) \in K[x]$ is a degree 3 polynomial. Recall that $C$ is a genus 1 curve. We specialise the construction of Definition 9 to $X$ :

Let $\alpha_{0}, \alpha_{1} \in H^{0}\left(X, \Omega_{X}^{1}\right)$ be 1-forms on $X$ with $\alpha_{0}$ regular on $C$ and $\alpha_{1}$ having a pole of order 2 at $\infty$ such that the cohomology classes of $\alpha_{0}, \alpha_{1}$ are a $K$-basis for $H_{d r}^{1}(X / K)$. Let $R_{n}$ and $\mathscr{U}_{n}$ be as in Definition 9.

Remark 17 It will be convenient at this stage to fix a choice of ordered basis $\mathscr{B}_{n}$ for $R_{n}$. We take as a $K$-basis the words of length less than or equal to $n$ with a graded lexicographic ordering such that $A_{0}<A_{1}<1$. With respect to this ordered basis we denote by $w_{l}^{k}$ the $k$-th word of length $l$. For example, the ordering on all words of length up to 2 is $w_{2}^{1}, w_{2}^{2}, w_{2}^{3}, w_{2}^{4}, w_{1}^{1}, w_{1}^{2}, w_{0}^{1}$.

Note that there are $2^{l}$ words of length $l$ and $\mathscr{B}_{n}$ has order $2^{n}+2^{n-1}+\cdots+1=2^{n+1}-1$. Thus if we have a word $w_{l}^{k}$, then $A_{0} w_{l}^{k}=w_{l+1}^{k}$ and $A_{1} w_{l}^{k}=w_{l+1}^{k+2^{l}}$. We can describe the action of $\nabla$ on a basis for $R_{n}$ :

$$
\nabla\left(w_{l}^{k}\right)= \begin{cases}-A_{0} w_{l}^{k} \alpha_{0}-A_{1} w_{l}^{k} \alpha_{1}=-w_{l+1}^{k} \alpha_{0}-w_{l+1}^{k+2^{l}} \alpha_{1} & \text { if } l \leq n-1 \\ 0 & \text { if } l=n\end{cases}
$$

Lemma 5 The connection matrix of $\mathscr{U}_{0}$ is the zero matrix. If $C_{n}$ is the connection matrix of $\mathscr{U}_{n}$ with respect to the basis $\mathscr{B}_{n}$, then

$$
C_{n+1}=\left(\begin{array}{cc}
0_{2^{n+1}} \times 2^{n+1} & D_{n+1} \\
0_{2^{n+1}-1 \times 2^{n+1}} & C_{n}
\end{array}\right)
$$

is the connection matrix of $\mathscr{U}_{n+1}$ with respect to the basis $\mathscr{B}_{n+1}$ where

$$
D_{n+1}=\left(\begin{array}{ll}
-\alpha_{0} I_{2^{n}} & 0_{2^{n} \times 2^{n}-1} \\
-\alpha_{1} I_{2^{n}} & 0_{2^{n} \times 2^{n}-1}
\end{array}\right) .
$$


Here $0_{r \times s}$ is the $r \times s$ null matrix and $I_{r}$ is the $r \times r$ identity matrix.

Proof This is just a straightforward calculation given Remark 17.

We now explicitly compute the extension of the $\mathscr{U}_{n}$ to logarithmic connections on $C$ by application of Algorithm 1. Recall that the logarithmic extension $\overline{\mathscr{U}}_{0}$ of $\mathscr{U}_{0}$ is defined to be $\left(\mathscr{O}_{C}, d\right)$. We now present an example calculation of the computation of the extension at level 1 .

Example 1 The ordered basis elements for $R_{1}$ are $\mathscr{B}_{1}=\left\{A_{0}, A_{1}, 1\right\}$. Then we have

$$
\begin{aligned}
\nabla\left(A_{0}\right) & =\nabla\left(A_{1}\right)=0 \\
\nabla(1) & =-A_{0} \alpha_{0}-A_{1} \alpha_{1}
\end{aligned}
$$

The connection matrix of $\nabla$ on $\mathscr{U}_{1}=R_{1} \otimes \mathscr{O}_{X}$ with respect to $\mathscr{B}_{1}$ is

$$
C_{1}=\left(\begin{array}{ccc}
0 & 0 & -\alpha_{0} \\
0 & 0 & -\alpha_{1} \\
0 & 0 & 0
\end{array}\right)
$$

There is a natural projection map $\pi_{1}: \mathscr{U}_{1} \rightarrow \mathscr{O}_{X}$. We wish to find an open $Y \subset C$ containing $\infty$, a connection $\mathscr{U}_{1}^{\prime}$ on $Y$, and a gauge transformation $G_{1}$ such that over $X \cap Y$ we have a commutative diagram

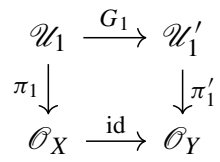

where $\pi_{1}^{\prime}$ is a projection map from $\mathscr{U}_{1}^{\prime} \rightarrow \mathscr{O}_{Y}$. In order to ensure that $G_{1}$ satisfies (4) it must be of the form

$$
G_{1}=\left(\begin{array}{ccc}
1 & 0 & h^{1} \\
0 & 1 & O h^{2} \\
0 & 0 & 1
\end{array}\right)
$$

where $h^{1}, h^{2} \in K(C)$. The gauge transformation of $C_{1}$ by $G_{1}$ is

$$
C_{1}^{\prime}=G_{1}^{-1} d G_{1}+G_{1}^{-1} C_{1} G_{1}=C_{1}+d G_{1}=\left(\begin{array}{ccc}
0 & 0 & -\alpha_{0}+d h^{1} \\
0 & 0 & -\alpha_{1}+d h^{2} \\
0 & 0 & 0
\end{array}\right)
$$

Choose $h^{1}=0$ and $h^{2}=F \in K(C)$ with a pole of order 1 at $\infty$ such that $-\alpha_{1}+d F$ is regular at $\infty$. Let $Y$ be the open set $\left\{P \in C: \alpha_{1}+d F\right.$ is regular at $\left.P\right\} \subset C$. Then we define $\mathscr{U}_{1}^{\prime}$ to be the connection on $Y$ defined with connection matrix $C_{1}^{\prime}$ and bundle $R_{1} \otimes \mathscr{O}_{Y}$. The logarithmic extension $\overline{\mathscr{U}}_{1}$ of $\mathscr{U}_{1}$ is then described by the descent datum of the logarithmic connections $\mathscr{U}_{1}$ (on $X$ ) and $\mathscr{U}_{1}^{\prime}$ (on $Y$ ) together with the gauge transformation $G_{1}:\left.\left.\mathscr{U}_{1}\right|_{X \cap Y} \stackrel{\sim}{\rightarrow} \mathscr{U}_{1}^{\prime}\right|_{X \cap Y}$.

Having dealt with the first non-trivial case we now turn our attention to computing the extension of $\mathscr{U}_{n+1}$ for general level. We will do this by showing that we can calculate a suitable gauge transformation $G_{n+1}$ given an extension $\overline{\mathscr{U}}_{n}$ defined at level $n$. As in Example 1 we find that $G_{n+1}$ should be of the form

$$
G_{n+1}=\left(\begin{array}{cc}
I_{2^{n+1}} & H_{n+1} \\
0 & G_{n}
\end{array}\right)
$$


where $H_{n+1}$ is some $2^{n+1} \times 2^{n+1}-1$ matrix over $K(C)$ which we need to determine. The bundle $\mathscr{U}_{n+1}^{\prime}$ should again be a trivial bundle $R_{n+1} \otimes \mathscr{O}_{Y}$, where we would like to choose $Y$ as in Example 1.

Lemma 6 Let $n \geq 1$ and let $C_{n}$ (resp. $\left.C_{n+1}\right)$ be the connection matrix of $\mathscr{U}_{n}\left(\right.$ resp. $\left.\mathscr{U}_{n+1}\right)$ with respect to the basis $\mathscr{B}_{n}$ (resp. $\mathscr{B}_{n+1}$ ). Suppose that $\mathscr{U}_{n}$ extends to a logarithmic connection $\overline{\mathscr{U}}_{n}$ described by a logarithmic connection $\mathscr{U}_{n}$ over $X$, a logarithmic connection $\mathscr{U}_{n}^{\prime}$ over some open $Y$ and a gauge transformation $G_{n}$ over $X \cap Y$. Suppose that $G_{n}$ takes $C_{n}$ to $C_{n}^{\prime}$, the connection matrix of $\mathscr{U}_{n}^{\prime}$. If $G_{n+1}$ is a gauge transformation of $C_{n+1}$ over $X \cap Y$ of the form

$$
G_{n+1}=\left(\begin{array}{cc}
I_{2^{n+1}} & H_{n+1} \\
0 & G_{n}
\end{array}\right)
$$

then the gauge transformation of the matrix $C_{n+1}$ of Lemma 5 by $G_{n+1}$ is

$$
C_{n+1}^{\prime}=G_{n+1}^{-1} d G_{n+1}+G_{n+1}^{-1} C_{n+1} G_{n+1}=\left(\begin{array}{cc}
0 & D_{n+1}^{\prime} \\
0 & C_{n}^{\prime}
\end{array}\right)
$$

where $D_{n+1}^{\prime}=D_{n+1} G_{n}+d H_{n+1}-H_{n+1} C_{n}^{\prime}$ and

$$
D_{n+1}=\left(\begin{array}{cc}
-\alpha_{0} I_{2^{n}} & 0_{2^{n} \times 2^{n}-1} \\
-\alpha_{1} I_{2^{n}} & 0_{2^{n} \times 2^{n}-1}
\end{array}\right) .
$$

Proof This follows easily from the definition of the gauge transformation and noting that

$$
G_{n+1}^{-1}=\left(\begin{array}{cc}
I_{2^{n+1}} & -H_{n+1} G_{n}^{-1} \\
0 & G_{n}^{-1}
\end{array}\right) .
$$

Remark 18 Note that in the above $C_{n}^{\prime}$ will be a matrix of 1-forms with at worst logarithmic poles at $\infty$ by the assumption that $\overline{\mathscr{U}}_{n}$ is logarithmic. Therefore, in order to compute a suitable gauge transformation $G_{n+1}$ we need to find a matrix of functions $H_{n+1}$ such that

$$
d H_{n+1}+D_{n+1} G_{n}-H_{n+1} C_{n}^{\prime}
$$

has entries with at worst logarithmic poles at $\infty$. This is the content of the following theorem.

In the course of the proof of the theorem we will need to make use of the following two auxiliary functions.

Definition 20 Let $\psi, \phi: \mathbb{Z}^{3} \times \mathrm{N} \mapsto \mathbb{Z}$ be the functions defined by

$$
\psi(r, i, j, k):= \begin{cases}\left\lfloor k^{i-j} r\right\rfloor+1 & \text { if } r \neq 00 \quad \bmod k^{j-i} \\ \left\lfloor k^{i-j} r\right\rfloor & \text { otherwise }\end{cases}
$$

and

$$
\phi(r, i, j, k):=(r-1) k^{i}-(\psi(r, i, j, k)-1) k^{j} .
$$

Remark 19 It is helpful at this stage to think about the functions $\phi$ and $\psi$ in terms words in the ordered basis $\mathscr{B}_{n}$ from Remark 17. Suppose that we have an alphabet with $k$-letters $A_{i}$ and let $w$ be the $r$-th word of length $j$ with respect to the lexicographic ordering $A_{0}<\cdots<$ $A_{k-1}<1$. Then for $i<j$ the first $i$ letters of $w$ are the $\psi(r, i, j, k)$-th word of length $i$ and the last $j-i$ letters of $w$ are the $\left(\phi(r, i, j, k) 2^{-i}+1\right)$-th word of length $j-i$. 
We are now ready to state and prove the following theorem.

Theorem 3 Let $C, X, \mathscr{U}_{n}$ and $C_{n}$ be as above. Then there is an open $Y \subset C$ containing $\infty$ such that for all $n$ there is an isomorphism $G_{n}$ of $\mathscr{U}_{n}$ with a connection $\mathscr{U}_{n}^{\prime}=\left(R_{n} \otimes \mathscr{O}_{Y}, d+\right.$ $\left.C_{n}^{\prime}\right)$ over $X \cap Y$ which is compatible with projection to lower level. The isomorphism $G_{n}$ can be chosen such that

$$
G_{n}=\left(\begin{array}{cc}
I_{2^{n}} & H_{n} \\
0 & G_{n-1}
\end{array}\right)
$$

where $H_{n}$ is a matrix of functions on $C$ and $C_{n}^{\prime}$ is a matrix of 1-forms on $C$ such that

$$
H_{n}=\left(\begin{array}{ccccc}
H_{n}^{1, n-1} & \ldots & H_{n}^{1, i} & \ldots & H_{n}^{1,0} \\
\vdots & & \vdots & & \vdots \\
H_{n}^{2, n-1} & \ldots & H_{n}^{2^{n-i}, i} & \ldots & H_{n}^{2^{n}, 0}
\end{array}\right) ; \quad C_{n}^{\prime}=\left(\begin{array}{ccccc}
C_{n}^{1, n} & \ldots & C_{n}^{1, i} & \ldots & C_{n}^{1,0} \\
\vdots & & \vdots & & \vdots \\
\vdots & \ldots & C_{n}^{2^{n+1-i}-1, i} & \ldots & C_{n}^{2^{n+1}-1,0} \\
C_{n}^{2, n} & \ldots & C_{n}^{2^{n+1-i}, i} & \ldots & C_{n}^{2^{n+1}, 0}
\end{array}\right)
$$

where

(A) For all $r, i$ there are rational functions $h_{n}^{r, i} \in K(C)$ such that $H_{n}^{r, i}=h_{n}^{r, i} I_{2^{i}}$

(B) For all $i$ we have

(B1) For all $r=1, \ldots, 2^{n+1-i}-2$ there are 1 -forms $c_{n+1}^{r, i} \in \Omega_{X / K}^{1}$ on $X$ with at worst logarithmic poles at $\infty$ such that $C_{n}^{r, i}=c_{n}^{r, i} I_{2^{i}}$

(B2) $C_{n}^{2^{n+1-i}-1, i}=0_{2^{j} \times 2^{j}}$

(B3) $C_{n}^{2^{n+1-i}, i}=0_{2^{i}-1 \times 2^{i}}$

Remark 20 Note that the matrices $H_{n}^{r, i}$ and $C_{n}^{r, i}$ have entries indexed by words. We can think of these two matrices in the following way: if I take a basis element coming from a word of length $i$ in $\mathscr{B}_{n}$ and apply the gauge transformation $G_{n}$ to it then $H_{n}^{r, i}$ gives the coefficients of the basis elements in the result coming from words of length $n$ whose first $n-i$ letters are the word with index $r$. Similarly, if I differentiate a basis element coming from a word of length $i$ in $\mathscr{B}_{n}$ using the connection $d+C_{n}^{\prime}$ then $C_{n}^{r, i}$ gives the coefficients of the basis elements in the result coming from words of length $n$ whose first $n-i$ letters are the word with index $r$.

Proof We shall proceed by induction and assume that the statement of the theorem holds for all $r \leq n$. In Example 1 we have already shown that this is true in the case that $n=1$ with $h_{1}^{1,0}=0$ and $h_{1}^{2,0}=F$. Let $Y$ be as in the aforementioned example.

In Lemma 6 we saw that $C_{n+1}^{\prime}$ is of the form

$$
\left(\begin{array}{cc}
0 & D_{n+1}^{\prime} \\
0 & C_{n}^{\prime}
\end{array}\right)
$$

where $D_{n+1}^{\prime}=d H_{n+1}+D_{n+1} G_{n}-H_{n+1} C_{n}^{\prime}$. By inductive hypothesis $C_{n}^{\prime}$ satisfies condition (B). Therefore, we must show that $H_{n+1}$ can be chosen such that

(i) $D_{n+1}^{\prime}$ has entries with at worst logarithmic poles at $\infty$

(ii) that $H_{n+1}$ satisfies condition (A)

and conclude then that $C_{n+1}^{\prime}$ satisfies $(B)$. 
We split $H_{n+1}$ and $C_{n+1}^{\prime}$ into block matrices

$$
\begin{aligned}
H_{n+1} & =\left(\begin{array}{ccccc}
H_{n+1}^{1, n} & \ldots & H_{n+1}^{1, i} & \ldots & H_{n}^{1,0} \\
\vdots & & \vdots & & \vdots \\
H_{n+1}^{2, n} & \ldots & H_{n+1}^{2^{n+1-i}, i} & \ldots & H_{n+1}^{2^{n+1}, 0}
\end{array}\right) ; \\
C_{n+1}^{\prime} & =\left(\begin{array}{ccccc}
C_{n+1}^{1, n+1} & \ldots & C_{n+1}^{1, i} & \ldots & C_{n+1}^{1,0} \\
\vdots & & \vdots & & \vdots \\
\vdots & \ldots & C_{n+2-1}^{2^{n+2-i}-1} & \ldots & C_{n+1}^{2^{n+2}-1,0} \\
C_{n+1}^{2, n+1} & \ldots & C_{n+1}^{2^{n+2}, i} & \ldots & C_{n+1}^{2^{n+2}, 0}
\end{array}\right)
\end{aligned}
$$

where each $H_{n+1}^{r, i}$ is a $2^{i} \times 2^{i}$ matrix; for $r=1, \ldots, 2^{n+2-i}-1$ each $C_{n+1}^{r, i}$ is a $2^{i} \times 2^{i}$ matrix; and, each $C_{n+1}^{2^{n+2-i}, i}$ is a $2^{i}-1 \times 2^{i}$ matrix. In order to complete the induction step we will try to express $C_{n+1}^{r, i}$ in terms of block matrices from $H_{n}, H_{n+1}$ and $C_{n}^{\prime}$.

It will be convenient to say that $H_{n+1}^{r, i}$ in $H_{n+1}$ stems from the block matrix $H_{n+1}^{s, j}$ for $i+1 \leq j \leq n$ if the rows of $H_{n+1}$ containing $H_{n+1}^{r, i}$ are a subset of the rows of $H_{n+1}$ containing $H_{n+1}^{s, j}$. It is not difficult then to see that $H_{n+1}^{r, i}$ in $H_{n+1}$ stems from the block matrix $H_{n+1}^{\psi(r, i, j, 2), j}$.

A simple calculation shows that

$$
D_{n+1} G_{n}=\left(\begin{array}{cc}
-\alpha_{0} I_{2^{n}} & -\alpha_{0} H_{n} \\
-\alpha_{1} I_{2^{n}} & -\alpha_{1} H_{n}
\end{array}\right)
$$

and by the inductive hypothesis we compute that the contribution of $d H_{n+1}+D_{n+1} G_{n}$ to $C_{n+1}^{r, i}$ is

$$
\begin{cases}d H_{n+1}^{r, n}-\alpha_{r-1} I_{2^{n}} & \text { if } i=n \\ d H_{n+1}^{r, i}-\alpha_{\psi(r, i, n, 2)-1} h_{n}^{r-(\psi(r, i, n, 2)-1) 2^{n-i}, i} I_{2^{i}} & \text { if } i<n .\end{cases}
$$

The contribution coming from $H_{n+1} C_{n}^{\prime}$ is more complicated to work out. We introduce a second inductive step to the argument, with our inductive hypothesis being that condition (A) is satisfied by $H_{n+1}$ for $i=n, n-1, \ldots, j+1$. We then show that this implies that condition (A) is satisfied by $H_{n+1}$ for $i=j$.

For our base case, we need to show that $H_{n+1}$ satisfies condition $(A)$ for $i=n$. It is straightforward, however, to see that

$$
H_{n+1} C_{n}^{\prime}=\left(\begin{array}{cc}
0_{2^{n} \times 2^{n}} * \\
0_{2^{n} \times 2^{n}} *
\end{array}\right)
$$

and so $C_{n+1}^{r, n}=d H_{n+1}^{r, n}-\alpha_{r-1} I_{2^{n}}$ for $r=1,2$. We choose $H_{n+1}^{r, n}=h_{n+1}^{r, n} I_{2^{n}}$ where $h_{n+1}^{r, n} \in$ $K(C)$ such that $d h_{n+1}^{r, n}-\alpha_{r-1}$ is regular at $\infty$. To ensure that we may continue to define the resulting logarithmic connection $\mathscr{U}_{n+1}^{\prime}$ over the same open $Y$ as $\mathscr{U}_{n}^{\prime}$, we choose $h_{n+1}^{r, n}$ to be polynomial in $h_{1}^{2,0}=F$. So we have that condition (A) holds for $i=n$.

Now let $j<n$ and assume then that condition (A) holds for $i=n, \ldots, j+1$. Now, rows $(r-1) 2^{j}+1$ to $(r-1) 2^{j}+2^{j}$ are the rows of $H_{n+1}$ containing $H_{n+1}^{r, j}$. They have the form

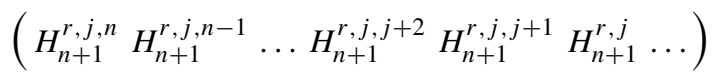


where each $H_{n+1}^{r, j, i}$ is a $2^{j} \times 2^{i}$ matrix. By the inductive hypothesis on $H_{n+1}^{r, i}$ we have the for $i=n, \ldots, j+1$

$$
H_{n+1}^{r, j, i}=\left(0_{2^{j} \times \phi(r, j, i, 2)} h_{n+1}^{\psi(r, j, i, 2), j} I_{2^{j}} 0_{2^{j} \times 2^{i}-\left(\phi(r, j, i, 2)+2^{j}\right)}\right) .
$$

Therefore we conclude that the contribution of $H_{n+1} C_{n}^{\prime}$ to $C_{n+1}^{r, j}$ is

$$
\begin{aligned}
& \left(H_{n+1}^{r, j, n} H_{n+1}^{r, j, n-1} \ldots H_{n+1}^{r, j, j+2} H_{n+1}^{r, j, j+1} H_{n+1}^{r, j} \ldots\right) \\
& \times\left(c_{n}^{1, j} I_{2^{j}} \ldots c_{n}^{2^{n+2-j}-2, j} I_{2^{j}} 0_{2^{j} \times 2^{j}} 0_{2^{j}-1 \times 2^{j}}\right)^{t} \\
& =\left(\sum_{t=j+1}^{n} c_{n}^{\left(2^{n}+2^{n-1}+\cdots+2^{t+1}+\phi(r, j, t, 2)\right) 2^{-j}+1, j} h_{n+1}^{\psi(r, j, t, 2), t}\right) I_{2^{j}} \\
& =\left(\sum_{t=j+1}^{n} c_{n}^{2^{n+1-j}-2^{t+1-j}+\phi(r, j, t, 2) 2^{-j}+1, j} h_{n+1}^{\psi(r, j, t, 2), t}\right) I_{2^{j}}
\end{aligned}
$$

This expression can be simplified further by noting the following: since we are assuming that $j<n$ the terms $c_{n}^{2^{n+1-j}-2^{t+1-j}+\phi(r, j, t, 2) 2^{-j}+1, j}$ appearing above can be replaced with $c_{m}^{k, j}$ for some $k, m$ with $m<n$. In particular, the original inductive hypothesis implies that if $r>2^{n-j}$ then $C_{n}^{r, j}=C_{n-1}^{r-2^{n-j}, j}$ and hence that $c_{n}^{r, j}=c_{n-1}^{r-2^{n-j}, j}$. Therefore, we conclude that we may replace each instance of $c_{n}^{2^{n+1-j}-2^{t+1-j}+\phi(r, j, t, 2) 2^{-j}+1, j}$ appearing above with $c_{t}^{\phi(r, j, t, 2) 2^{-j}+1, j}$. Then we may express $C_{n+1}^{r, j}$ as

$C_{n+1}^{r, j}=d H_{n+1}^{r, j}-\left(\alpha_{\psi(r, j, n, 2)-1} h_{n}^{r-(\psi(r, j, n, 2)-1) 2^{n-j}, j}+\sum_{t=j+1}^{n} c_{t}^{\phi(r, j, t, 2) 2^{-j}+1, j} h_{n+1}^{\psi(r, j, t, 2), t}\right) I_{2^{n}}$

The bracketed expression is a 1-form on the curve $C$, and so we can choose $h_{n+1}^{r, j} \in K(C)$ polynomial in $h_{1}^{2,0}=F$ such that

$$
d h_{n+1}^{r, j}-\alpha_{\psi(r, j, n, 2)-1} h_{n}^{r-(\psi(r, j, n, 2)-1) 2^{n-j}, i}-\sum_{t=j+1}^{n} c_{t}^{\phi(r, j, t, 2) 2^{-j}+1, i} h_{n+1}^{\psi(r, j, t, 2), t}
$$

has at worst logarithmic poles at $\infty$ and which has no other poles on $Y$. Take $H_{n+1}^{r, j}:=h_{n+1}^{r, j} I_{2^{j}}$. Repeating this for $r=1, \ldots, 2^{n+1-j}$ we see that condition $(A)$ is satisfied for $H_{n+1}$ for $i=j$. Hence, by induction condition (A) is satisfied by $H_{n+1}$. The construction also makes it clear that condition (B) is satisfied by $C_{n+1}^{\prime}$ with $c_{n+1}^{r, i}:=(7)$.

Now let $\mathscr{U}_{n+1}^{\prime}$ be the logarithmic connection on $Y$ with bundle $R_{n+1} \otimes \mathscr{O}_{Y}$ and connection $d+C_{n+1}^{\prime}$. By induction we conclude that for each $n$ there is an isomorphism $G_{n}$ from $\mathscr{U}_{n}$ to $\mathscr{U}_{n}^{\prime}$ over $Y_{\infty}$ such that the connection matrix of $\mathscr{U}_{n}^{\prime}$ is logarithmic on $Y$ along $\infty$. For each $n$ the logarithmic extension $\overline{\mathscr{U}}_{n}$ of $\mathscr{U}_{n}$ is described, therefore, by the descent datum of the logarithmic connection $\mathscr{U}_{n}$ over $X$, the logarithmic connection $\mathscr{U}_{n}^{\prime}$ over $Y$ and the isomorphism $G_{n}:\left.\left.\mathscr{U}_{n}\right|_{X \cap Y} \stackrel{\sim}{\rightarrow} \mathscr{U}_{n}^{\prime}\right|_{X \cap Y}$.

The following lemma shows how we may simplify the computations involved in application of the above theorem. 
Lemma 7 Let $C, D$ and $X$ be as above. Let $n \geq 1$ and let $\mathscr{U}_{n}$ be the $n$-th level finite quotient of the universal connection $\mathscr{U}$ on $X$. Let $\mathscr{U}_{n}^{\prime}$ be the connection and $G_{n}$ the isomorphism defined in Theorem 3. Then the functions $h_{n}^{r, i}$ may be chosen such that for $i \geq 0$ we have $h_{n+1}^{r, i+1}=h_{n}^{r, i}$ and $c_{n+1}^{r, i+1}=c_{n}^{r, i}$.

Proof This is a simple induction argument on noting that $\psi(r, i, j, 2)=\psi(r, i-1, j-1,2)$ and $\phi(r, i, j, 2)=2 \phi(r, i-1, j-1,2)$ and using condition (7) in the proof of Theorem 3.

Remark 21 Theorem 3 demonstrates that we may iteratively compute suitable logarithmic extensions of the universal connection $\mathscr{U}$ on $X$ by computing compatible logarithmic extensions of the finite level quotients $\mathscr{U}_{n}$. Lemma 7 simplifies the computations involved in computing the connection matrices and gauge transformations. Assume we have computed the extension $\overline{\mathscr{U}}_{n}$ in the form of the datum $\mathscr{U}_{n}, \mathscr{U}_{n}^{\prime}$ and $G_{n}$. Then we simply need to determine what the image of 1 at level $n+1$ under the gauge transformation $G_{n+1}$ should be. By Lemma 7 this immediately determines a suitable gauge transformation. That is, at level $n+1$ for each $r=1, \ldots, 2^{n}$ we need to compute a $h_{n+1}^{r, 0} \in K(C)\left(\right.$ polynomial in $F=h_{1}^{2,0}$ ) such that

$$
d h_{n+1}^{r, 0}-\alpha_{\psi(r, 0, n, 2)-1} h_{n}^{r-(\psi(r, 0, n, 2)-1) 2^{n}, 0}-\sum_{t=1}^{n} c_{t}^{\phi(r, 0, t, 2)+1,0} h_{n+1}^{\psi(r, 0, t, 2), t}
$$

has at worst logarithmic poles at $\infty$. By expanding (8) in a local parameter $t$ at $\infty$, we can compute $h_{n+1}^{r, 0}$ locally as the formal integral of

$$
\sum_{t=1}^{n} c_{t}^{\phi(r, 0, t, 2)+1,0} h_{n+1}^{\psi(r, 0, t, 2), t}+\alpha_{\psi(r, 0, n, 2)-1} h_{n}^{r-(\psi(r, 0, n, 2)-1) 2^{n}, 0} .
$$

In the following algorithm we compute the gauge transformations iteratively.

Algorithm 2 (Computing the logarithmic extension of $\mathscr{U}_{n}$ on elliptic curves) Input:

- Elliptic curve $C$ over a characteristic 0 field $K$ with affine model $X$ of the form $y^{2}=f(x)$ with $f(x) \in K[x]$ and $\operatorname{deg} f=3$.

- Universal connection $\mathscr{U}=\left\{\left(\mathscr{U}_{n}, 1\right)\right\}$ on $X$ with respect to the basis $\alpha_{0}, \alpha_{1}$ of $H_{d r}^{1}(X)$ as at the beginning of Sect. 3.2.

- The connection matrix $C_{n}^{\prime}$ over open $Y \subset C$ of the logarithmic extension $\overline{\mathscr{U}}_{n}$ of $\mathscr{U}_{n}$.

- The gauge transformation $G_{n}$ defining the extension $\overline{\mathscr{U}}_{n}$ with respect to the basis $\mathscr{B}_{n}$.

Output:

- The connection matrix $C_{n+1}^{\prime}$ over open $Y \subset C$ of the logarithmic extension $\overline{\mathscr{U}}_{n+1}$ of $\mathscr{U}_{n+1}$.

- The gauge transformation $G_{n+1}$ defining the extension $\overline{\mathscr{U}}_{n+1}$ with respect to the basis $\mathscr{B}_{n+1}$.

Algorithm:

I If $n=0$ then

(1) Take $h_{1}^{1,0} \in K(C)$ regular at $\infty$.

(2) Take $h_{1}^{2,0} \in K(C)$ with a single simple pole at $\infty$ such that $d h_{1}^{2,0}-\alpha_{1}$ has at worst a logarithmic pole at $\infty$. 
(3) Define

$$
\begin{aligned}
G_{1} & =\left(\begin{array}{ccc}
1 & 0 & h_{1}^{1,0} \\
0 & 1 & h_{1}^{2,0} \\
0 & 0 & 1
\end{array}\right) \\
C_{1}^{\prime} & =\left(\begin{array}{ccc}
0 & 0 & -\alpha_{0}+d h_{1}^{1,0} \\
0 & 0 & -\alpha_{1}+d h_{1}^{2,0} \\
0 & 0 & 0
\end{array}\right)=\left(\begin{array}{ccc}
0 & 0 & c_{1}^{1,0} \\
0 & 0 & c_{1}^{2,0} \\
0 & 0 & 0
\end{array}\right) .
\end{aligned}
$$

II Else

(1) For $0<i \leq n, 1 \leq r \leq 2^{n+1-i}$ define:
i. $h_{n+1}^{r, i}:=h_{n}^{r, i=1}$
ii. $c_{n+1}^{r, i}:=c_{n}^{r, i-1}$

(2) For $i=0,1 \leq r \leq 2^{n+1}$ take $h_{n+1}^{r, 0} \in K(C)$ polynomial in $h_{1}^{2,0}$ such that

$$
d h_{n+1}^{r, 0}-\alpha_{\psi(r, 0, n, 2)-1} h_{n}^{r-(\psi(r, 0, n, 2)-1) 2^{n}, 0}-\sum_{t=1}^{n} c_{t}^{\phi(r, 0, t, 2)+1,0} h_{n+1}^{\psi(r, 0, t, 2), t}
$$

has at worst logarithmic poles at $\infty$. Define $c_{n+1}^{r, 0}:=(9)$.

(3) For $0 \leq i \leq n, 1 \leq r \leq 2^{n+1-i}$ let $H_{n+1}^{r, i}:=h_{n+1}^{r, i} I_{2^{i}}$. Define $H_{n+1}$ to be the matrix

$$
H_{n+1}=\left(\begin{array}{ccccc}
H_{n+1}^{1, n} & \ldots & H_{n+1}^{1, i} & \ldots & H_{n}^{1,0} \\
\vdots & & \vdots & & \vdots \\
H_{n+1}^{2, n} & \ldots & H_{n+1}^{2^{n+1-i}, i} & \ldots & H_{n+1}^{2^{n+1}, 0}
\end{array}\right)
$$

and define

$$
G_{n+1}:=\left(\begin{array}{cc}
I & H_{n+1} \\
0 & G_{n}
\end{array}\right)
$$

(4) For $0 \leq i \leq n, 1 \leq r \leq 2^{n+1-i}$ let $C_{n+1}^{r, i}:=c_{n+1}^{r, i} I_{2^{i}}$. Define $D_{n+1}^{\prime}$ to be the matrix

$$
D_{n+1}^{\prime}=\left(\begin{array}{ccccc}
C_{n+1}^{1, n} & \ldots & C_{n+1}^{1, i} & \ldots & C_{n+1}^{1,0} \\
\vdots & & \vdots & & \vdots \\
C_{n+1}^{2, n} & \ldots & C_{n+1}^{2^{n+1-i}, i} & \ldots & C_{n+1}^{2^{n+1}, 0}
\end{array}\right)
$$

and define

$$
C_{n+1}^{\prime}:=\left(\begin{array}{cc}
0 & D_{n+1}^{\prime} \\
0 & C_{n}^{\prime}
\end{array}\right)
$$

We end this section with an application of Algorithm 2 to successively compute the extension of $\mathscr{U}_{n}$ on $X$ to $\overline{\mathscr{U}}_{n}$ on $C$ for $n=2,3$ and 4. For brevity, we describe only the gauge transformations $G_{n}$ computed by the algorithm and in level 4 we only describe the image of 1 under the gauge transformation $G_{4}$. However, using the above this is clearly sufficient to describe the extensions. 
Proposition 4 The gauge transformation $G_{2}$ computed by Algorithm 2 and extending $G_{1}$ as computed in Example 1 is

$$
G_{2}=\left(\begin{array}{cc}
I & H_{2} \\
0 & G_{1}
\end{array}\right), \quad H_{2}=\left(\begin{array}{ccc}
0 & 0 & 0 \\
0 & 0 & 0 \\
F & 0 & 0 \\
0 & F & \frac{1}{2} F^{2}
\end{array}\right) .
$$

Proof In Example 1 we computed the extension of $\mathscr{U}_{1}$ to $\overline{\mathscr{U}}_{1}$ and saw that we may define

$$
G_{1}=\left(\begin{array}{ccc}
1 & 0 & 0 \\
0 & 1 & F \\
0 & 0 & 1
\end{array}\right)
$$

for some $F \in K(C)$ with a simple pole at $\infty$. In the notation of Theorem 3 we have

$$
\begin{aligned}
& h_{1}^{1,0}=0, \quad h_{1}^{2,0}=F \\
& c_{1}^{1,0}=-\alpha_{0}, \quad c_{1}^{2,0}=-\alpha_{1}+d F .
\end{aligned}
$$

Applying Algorithm 2 with $n=1$ then as in Step II.1) we define

$$
\begin{aligned}
& h_{2}^{1,1}=0, \quad h_{2}^{2,1}=F \\
& c_{2}^{1,1}=-\alpha_{0}, \quad c_{2}^{2,1}=-\alpha_{1}+d F .
\end{aligned}
$$

Following Step (II)(2) for $r=1,2,3,4$ we need to compute $h_{2}^{r, 0}$ polynomial in $F$ such that

$$
c_{2}^{r, 0}=d h_{2}^{r, 0}-\alpha_{\psi(r, 0,1,2)-1} h_{1}^{r-(\psi(r, 0,1,2)-1) 2,0}-c_{1}^{\phi(r, 0,1,2)+1,0} h_{2}^{\psi(r, 0,1,2), 1}
$$

has at worst logarithmic poles at $\infty$. Here we find that

$$
\begin{aligned}
& r=1: c_{2}^{1,0}=d h_{2}^{1,0} \\
& r=2: c_{2}^{2,0}=d h_{2}^{2,0}-F \alpha_{0} \\
& r=3: c_{2}^{3,0}=d h_{2}^{3,0}+F \alpha_{0} \\
& r=4: c_{2}^{4,0}=d h_{2}^{4,0}-F \alpha_{1}-\left(-\alpha_{1}+d F\right) F=d h_{2}^{4,0}-F d F .
\end{aligned}
$$

Since $\alpha_{0}$ is regular at $\infty$ and $F$ has a simple pole there we can take $h_{2}^{1,0}=h_{2}^{2,0}=h_{2}^{3,0}=0$ and also take $h_{2}^{4,0}=\frac{1}{2} F^{2}$. Therefore, we find that we can take the matrix $G_{2}$ to be

$$
G_{2}=\left(\begin{array}{cc}
I & H_{2} \\
0 & G_{1}
\end{array}\right), \quad H_{2}=\left(\begin{array}{ccc}
0 & 0 & 0 \\
0 & 0 & 0 \\
F & 0 & 0 \\
0 & F & \frac{1}{2} F^{2}
\end{array}\right)
$$

and then $C_{2}^{\prime}$, the gauge transformation of $C_{2}$ by $G_{2}$ is

$$
C_{2}^{\prime}=\left(\begin{array}{ll}
0 & D_{2}^{\prime} \\
0 & C_{1}^{\prime}
\end{array}\right), \quad D_{2}^{\prime}=\left(\begin{array}{ccc}
-\alpha_{0} & 0 & 0 \\
0 & -\alpha_{0} & -F \alpha_{0} \\
\alpha_{1}^{\prime} & 0 & F \alpha_{0} \\
0 & \alpha_{1}^{\prime} & 0
\end{array}\right)
$$

where $\alpha_{1}^{\prime}=-\alpha_{1}+d F$. 
Proposition 5 The gauge transformation $G_{3}$ computed by Algorithm 2 and extending $G_{2}$ as computed in Proposition 4 is

$$
G_{3}=\left(\begin{array}{cc}
I_{8 \times 8} & H_{4} \\
0_{7 \times 8} & G_{2}
\end{array}\right), \quad H_{3}=\left(\begin{array}{ccccccc}
0 & 0 & 0 & 0 & 0 & 0 & 0 \\
0 & 0 & 0 & 0 & 0 & 0 & 0 \\
0 & 0 & 0 & 0 & 0 & 0 & 0 \\
0 & 0 & 0 & 0 & 0 & 0 & \lambda F \\
F & 0 & 0 & 0 & 0 & 0 & 0 \\
0 & F & 0 & 0 & 0 & 0 & -2 \lambda F \\
0 & 0 & F & 0 & \frac{1}{2} F^{2} & 0 & \lambda F \\
0 & 0 & 0 & F & 0 & \frac{1}{2} F^{2} & \frac{1}{6} F^{3}
\end{array}\right)
$$

where $\lambda \in K$ is such that $\lambda d F-\frac{1}{2} F^{2} \alpha_{0}$ has a logarithmic pole at $\infty$.

Proof This is a straightforward application of Algorithm 2 making use of the extension $\overline{\mathscr{U}}_{2}$ computed in Proposition 4.

Proposition 6 The gauge transformation $G_{4}$ computed by Algorithm 2 and extending $G_{2}$ as computed in Proposition 5 is such that

$$
\begin{aligned}
G_{4}(1)= & \left(\frac{1}{6} \lambda F^{2}+\mu F\right) A_{0} A_{1}^{3}+\left(\frac{1}{2} \lambda F^{2}-3 \mu F\right) A_{1} A_{0} A_{1}^{2}+\left(3 \mu F-\frac{3}{2} \lambda F^{2}\right) A_{1}^{2} A_{0} A_{1} \\
& +\left(\frac{5}{6} \lambda F^{2}-\mu F\right) A_{1}^{3} A_{0}+\frac{1}{24} F^{4} A_{1}^{4}+\text { words of length } \leq 3
\end{aligned}
$$

where $\mu \in K$ is such that $\frac{1}{3} \lambda F d F+\mu d F-\frac{1}{6} F^{3} \alpha_{0}$ has at worst a simple pole at $\infty$.

Proof Again this is simply an application of Algorithm 2 lifting the extension computed in Proposition 5.

Remark 22 We may question whether the constants $\lambda$ and $\mu$ have any dependence on the choice of $F$. In fact they depend only on the choice of differentials $\alpha_{0}, \alpha_{1}$ as can be seen by expanding the differentials and $F$ in a local parameter at $\infty$. It is a simple calculation then to verify the independence from $F$.

\subsection{Logarithmic extensions on affine hyperelliptic curves}

Let $C$ be an odd hyperelliptic curve of genus $g \geq 2$ over a field $K$ of characteristic 0 . Say that we have an affine model of $C$ of the form

$$
y^{2}=f(x), f(x) \in K[x]
$$

with $\operatorname{deg}(f)=2 g+1$. There is a single $K$-rational point $\infty$ at infinity. Let $X$ be the affine curve $y^{2}=f(x)$ over $K$ so that $X=C-\{\infty\}$. Then $H_{d r}^{1}(X / K)$ has a $K$-basis of size $2 g$. Specialise Definition 9 to $X$, taking $\alpha_{0}, \alpha_{1}, \ldots \alpha_{2 g-1} \in H^{0}\left(X, \Omega_{X}^{1}\right)$ be 1-forms on $X$ such that their cohomology classes form a $K$-basis for $H_{d r}^{1}(X / K)$. Since $C$ is of genus $g$, we may further assume that $\alpha_{0}, \ldots, \alpha_{g-1}$ is a $K$-basis of $H^{0}\left(C, \Omega_{C}^{1}\right)$.

As in Sect. 3.2 we take as a basis $\mathscr{B}_{n}$ for $R_{n}$ the one given by the graded lexicographic order such that $A_{0}<A_{1}<\cdots<A_{2 g-1}<1$. If the $k$-th word of length $l$ is $w_{l}^{k}$ with respect to this basis then we easily conclude that $A_{i} w_{l}^{k}=w_{l+1}^{(2 g)^{l} i+k}$. So we can describe the action of $\nabla$ on a basis for $R_{n}$ : 


$$
\nabla\left(w_{l}^{k}\right)= \begin{cases}-\sum_{i=0}^{2 g-1} A_{i} w_{l}^{k} \alpha_{i}=-\sum_{i=0}^{2 g-1} w_{l+1}^{(2 g)^{l} i+k} \alpha_{i} & \text { if } l \leq n-1 \\ 0 & \text { if } l=n\end{cases}
$$

Given this it is simple to prove the following lemma.

Lemma 8 The connection matrix of $\mathscr{U}_{0}$ is the zero matrix. If $C_{n}$ is the connection matrix of $\mathscr{U}_{n}$ with respect to the basis $\mathscr{B}_{n}$, then

$$
C_{n+1}=\left(\begin{array}{cc}
0_{(2 g)^{n+1}} \times(2 g)^{n+1} & D_{n+1} \\
0_{\frac{(2 g)^{n+1}-1}{2 g-1} \times(2 g)^{n+1}} & C_{n}
\end{array}\right)
$$

is the connection matrix of $\mathscr{U}_{n+1}$ with respect to the basis $\mathscr{B}_{n+1}$ where

$$
D_{n+1}=\left(\begin{array}{cc}
-\alpha_{0} I_{(2 g)^{n}} & 0 \\
\vdots & \vdots \\
-\alpha_{2 g-1} I_{(2 g)^{n}} & 0
\end{array}\right) .
$$

It is straightforward to prove the analogous versions of Theorem 3 and Lemma 7 for odd models of hyperelliptic curves. Hence we may iteratively compute the logarithmic extensions of $\mathscr{U}_{n}$ using the following algorithm:

Algorithm 3 (Computing the logarithmic extension of $\mathscr{U}_{n}$ on hyperelliptic curves) Input:

- Hyperelliptic curve $C$ over a characteristic 0 field $K$ of genus $g$ with affine model $X$ of the form $y^{2}=f(x)$ with $f(x) \in K[x]$ and $\operatorname{deg} f=2 g+1, F \in K(C)$ fixed with a pole of order 1 at $\infty$.

- Universal connection $\mathscr{U}=\left\{\left(\mathscr{U}_{n}, 1\right)\right\}$ on $X$ with respect to the basis $\alpha_{0}, \ldots, \alpha_{2 g-1}$ of $H_{d r}^{1}(X)$ as above

- The connection matrix $C_{n}^{\prime}$ over open $Y \subset C$ of the logarithmic extension $\overline{\mathscr{U}}_{n}$ of $\mathscr{U}_{n}$.

- The gauge transformation $G_{n}$ defining the extension $\overline{\mathscr{U}}_{n}$ with respect to the basis $\mathscr{B}_{n}$.

Output:

- The connection matrix $C_{n+1}^{\prime}$ over open $Y \subset C$ of the logarithmic extension $\overline{\mathscr{U}}_{n+1}$ of $\mathscr{U}_{n+1}$.

- The gauge transformation $G_{n+1}$ defining the extension $\overline{\mathscr{U}}_{n+1}$ with respect to the basis $\mathscr{B}_{n+1}$.

Algorithm:

I If $n=0$ then

(1) For $r=1, \ldots, 2 g$ take $h_{1}^{r, 0} \in K(C)$ polynomial in $F$ such that $d h_{1}^{r, 0}-\alpha_{r-1}$ has at worst a logarithmic pole at $\infty$.

(2) For $r=1, \ldots, 2 g$ define $c_{1}^{r, 0}:=d h_{1}^{r, 0}-\alpha_{r-1}$

(3) Define

$$
\begin{gathered}
G_{1}=\left(\begin{array}{cc}
I_{2 g} & H_{1} \\
0 & 1
\end{array}\right), \quad H_{1}=\left(\begin{array}{c}
d h_{1}^{1,0} \\
\vdots \\
d h_{1}^{r, 0}
\end{array}\right) \\
C_{1}^{\prime}=\left(\begin{array}{cc}
0 & D_{1} \\
0 & 0
\end{array}\right), \quad D_{1}=\left(\begin{array}{c}
c_{1}^{1,0} \\
\vdots \\
c_{1}^{2 g, 0}
\end{array}\right) .
\end{gathered}
$$


II Else

(1) For $0<i \leq n, 1 \leq r \leq(2 g)^{n+1-i}$ define:
i. $h_{n+1}^{r, i}:=h_{n}^{r, i=}$
ii. $c_{n+1}^{r, i}:=c_{n}^{r, i-1}$

(2) For $i=0,1 \leq r \leq(2 g)^{n+1}$ take $h_{n+1}^{r, 0} \in K(C)$ polynomial in $F$ such that

$$
d h_{n+1}^{r, 0}-\alpha_{\psi(r, 0, n, 2 g)-1} h_{n}^{r-(\psi(r, 0, n, 2 g)-1)(2 g)^{n}, 0}-\sum_{t=1}^{n} c_{t}^{\phi(r, 0, t, 2 g)+1,0} h_{n+1}^{\psi(r, 0, t, 2 g), t}
$$

has at worst logarithmic poles at $\infty$. Define $c_{n+1}^{r, 0}:=(10)$.

(3) For $0 \leq i \leq n, 1 \leq r \leq(2 g)^{n+1-i}$ let $H_{n+1}^{r, i}:=h_{n+1}^{r, i} I_{(2 g)}$. Define $H_{n+1}$ to be the matrix

$$
H_{n+1}=\left(\begin{array}{ccccc}
H_{n+1}^{1, n} & \ldots & H_{n+1}^{1, i} & \ldots & H_{n}^{1,0} \\
\vdots & & \vdots & & \vdots \\
H_{n+1}^{2 g, n} & \ldots & H_{n+1}^{(2 g)^{n+1-i}, i} & \ldots & H_{n+1}^{(2 g)^{n+1}, 0}
\end{array}\right)
$$

and define

$$
G_{n+1}:=\left(\begin{array}{cc}
I & H_{n+1} \\
0 & G_{n}
\end{array}\right)
$$

(4) For $0 \leq i \leq n, 1 \leq r \leq(2 g)^{n+1-i}$ let $C_{n+1}^{r, i}:=c_{n+1}^{r, i} I_{(2 g)}$. Define $D_{n+1}^{\prime}$ to be the matrix

$$
D_{n+1}^{\prime}=\left(\begin{array}{ccccc}
C_{n+1}^{1, n} & \ldots & C_{n+1}^{1, i} & \ldots & C_{n+1}^{1,0} \\
\vdots & & \vdots & & \vdots \\
C_{n+1}^{2 g, n} & \ldots & C_{n+1}^{(2 g)^{n+1-i}, i} & \ldots & C_{n+1}^{(2 g)^{n+1}, 0}
\end{array}\right)
$$

and define

$$
C_{n+1}^{\prime}:=\left(\begin{array}{cc}
0 & D_{n+1}^{\prime} \\
0 & C_{n}^{\prime}
\end{array}\right)
$$

Remark 23 Suppose that we allow $g=1$ in the above algorithm. Then we recover the steps found in Algorithm 2 and we may take Algorithm 3 as a general algorithm to compute universal logarithmic extensions of $\mathscr{U}_{n}$ for elliptic curves or odd hyperelliptic curves.

We conclude this section with one final result which will be of use to us in the next section. This will give us an explicit description of how the gauge transformation $G_{n}$ acts on a basis $\mathscr{B}_{n}$ for $R_{n}$, the words in $A_{0}, \ldots, A_{2 g-1}$ of length at most $n$.

Lemma 9 Let $\mathscr{B}_{n}$ be the basis of $R_{n}$ consisting of words in $A_{0}, \ldots, A_{2 g-1}$ of length at most $n$ ordered with the graded lexicographic ordering such that $A_{i}<A_{j}$ if $i<j$. Let $G_{n}$ be the gauge transformation computed by Algorithm 3. Then $G_{n}$ acts on $w_{l}^{k}$ as follows:

$$
G_{n}: w_{l}^{k} \mapsto \begin{cases}w_{l}^{k} & \text { if } l=n \\ w_{l}^{k}+\sum_{s=l+1}^{n} \sum_{t=1}^{(2 g)^{s-l}} w_{s}^{k+(t-1)(2 g)^{l}} h_{s}^{t, l} & \text { otherwise. }\end{cases}
$$


Proof Suppose first that $l=n$. Then by construction we have that $G_{n}\left(w_{n}^{k}\right)=w_{n}^{k}$ for all $k$. Otherwise, suppose that $l<n$. Using Algorithm $3 G_{n}$ will be of the form

$$
G_{n}=\left(\begin{array}{cc}
I & H_{n} \\
0 & G_{n-1}
\end{array}\right), \quad H_{n}=\left(\begin{array}{ccccc}
h_{n}^{1, n-1} I_{(2 g)^{n-1}} & \ldots & h_{n}^{1, i} I_{(2 g)^{i}} & \ldots & h_{n}^{1,0} I_{1} \\
\vdots & \vdots & \vdots \\
h_{n}^{2 g, n-1} I_{(2 g)^{n-1}} & \ldots & h_{n}^{(2 g)^{n-i}, i} I_{(2 g) i} & \ldots & h_{n}^{(2 g)^{n}, i} I_{1}
\end{array}\right) .
$$

The word $w_{l}^{k}$ will correspond to column $(2 g)^{n}+(2 g)^{n-1}+\cdots+(2 g)^{l+1}+k$ in the matrix $G_{n}$. The submatrix $H_{n}$ will, therefore, contribute

$$
h_{n}^{1, l} w_{n}^{k}+h_{n}^{2, l} w_{n}^{k+(2 g)^{l}}+\cdots+h_{n}^{(2 g)^{n-l}, l} w_{n}^{k+\left((2 g)^{n-l}-1\right)(2 g)^{l}, l} .
$$

By a simple induction argument the lemma then follows.

\section{The Hodge filtration on $\mathscr{U}$}

\subsection{Computation of the Hodge filtration}

In this section we shall utilise the computations of Sect. 3 to provide an explicit version of results due to Hadian in [21]. Hadian provides a characterisation of the Hodge filtration on the universal connection $\mathscr{U}$ of $X:=C-D$, where $C$ is a general smooth projective curve of genus $g$ over a field $K$ of characteristic 0 and $D$ is a non-empty divisor defined over $K$ of size $r$. Fix a basepoint $b \in X(K)$.

Definition 21 By a filtered logarithmic connection $\mathscr{V}:=\left(\mathscr{V}, \nabla, F^{\bullet}\right)$ on $C$ with $\log$ poles along $D$ we mean a vector bundle $\mathscr{V}$ with a logarithmic connection $\nabla$ with $\log$ poles along $D$ which is equipped with a decreasing filtration by sub-bundles

$$
\mathscr{V}=F^{m} \mathscr{V} \subset F^{m+1} \mathscr{V} \subset \cdots \subset F^{n} \mathscr{V}=0
$$

for some $m<n \in \mathbb{Z}$ satisfying the Griffiths transversality property:

$$
\nabla\left(F^{i} \mathscr{V}\right) \subset F^{i-1} \mathscr{V} \otimes \Omega_{C / K}^{1}(D)
$$

for all $i$.

Remark 24 Note that the trivial connection $\mathscr{O}_{C}=\left(\mathscr{O}_{C}, d\right)$ is given the trivial filtration $F^{0}\left(\mathscr{O}_{C}\right)=\mathscr{O}_{C}, F^{1}\left(\mathscr{O}_{C}\right)=0$.

Definition 22 Let $\mathscr{U}, \mathscr{V}, \mathscr{W}$ be filtered logarithmic connections. An exact sequence of logarithmic connections

$$
0 \rightarrow \mathscr{U} \rightarrow \mathscr{V} \rightarrow \mathscr{W} \rightarrow 0
$$

is an exact sequence of filtered logarithmic connections if for each $p$ we have an exact sequence of sub-bundles

$$
0 \rightarrow F^{p} \mathscr{U} \rightarrow F^{p} \mathscr{V} \rightarrow F^{p} \mathscr{W} \rightarrow 0
$$

The dual space $V_{d r}$ in Definition 9 has a Hodge filtration induced by the natural trivial filtration on the de Rham complex of $X / K$, and this in turn induces a Hodge filtration on $V_{d r}^{\otimes n}$. Recall that $V_{d r}$ has basis $A_{0}, \ldots, A_{2 g+r-2}$ dual to differentials $\alpha_{0}, \ldots, \alpha_{2 g+r-2}$. We 
assume that the $\alpha_{i}$ are ordered so that $\alpha_{0}, \ldots, \alpha_{g-1}$ form a $K$-basis for $H^{0}\left(C, \Omega_{C / K}^{1}\right)$. As with elliptic and odd hyperelliptic curves we take as a $K$-basis of $V_{d r}^{\otimes n}$ the set $\mathscr{B}_{n}$ of words of length $n$ in $A_{0}, \ldots, A_{2 g+r-2}$ with graded lexicographic ordering such that $A_{i}<A_{j}$ if $i<j$.

Definition 23 The filtration $F^{\bullet}$ on $V_{d r}^{\otimes n}$ is defined as follows:

For $p>0$

$$
F^{p} V_{d r}^{\otimes n}:=0
$$

For $p<0$ we let $\tilde{F}^{p}:=\left\{w \in \mathscr{B}_{n}: w\right.$ contains at most $|p|$ occurrences of $\left.A_{0}, \ldots, A_{g-1}\right\}$. Then

$$
F^{p} V_{d r}^{\otimes n}:=\operatorname{Span}_{K}\left(\tilde{F}^{p}\right) .
$$

The natural filtration of $\mathscr{O}_{C}$ given by $F^{0} \mathscr{O}_{C}=\mathscr{O}_{C}$ and $F^{1} \mathscr{O}_{C}=0$ then together induce a filtration on $V_{d r}^{\otimes n} \otimes \mathscr{O}_{C}$ via the tensor product filtration. We may now state Hadian's Lemma in its full generality for $C$.

Lemma 10 ([21], Lemma 3.6) Let $C$ be a smooth projective curve over a field $K$ of characteristic $0, D$ a non-empty divisor and $X:=C-D$ and take $b \in X(K)$ a rational basepoint. Let $V_{d r}:=H_{d r}^{1}(X)^{\vee}$ and let $\mathscr{U}_{n}$ be the $n$-th finite level quotient of the universal connection on $X$ with respect to the basepoint $b$. Let $\overline{\mathscr{U}}_{n}$ be the extension of this to a logarithmic connection on $C$. Then there exists a filtration $\left(F^{\bullet} \overline{\mathscr{U}}_{n}\right)$ of vector bundles such that

(i) For all $n$ the filtration $F^{\bullet}$ on $\overline{\mathscr{U}}_{n}$ satisfies Griffiths transversality giving $\overline{\mathscr{U}}_{n}$ the structure of a filtered logarithmic connection. This filtration is unique up to automorphism of filtered logarithmic connections.

(ii) For all $n$ the exact sequence of logarithmic connections

$$
0 \rightarrow V_{d r}^{\otimes n} \otimes \mathscr{O}_{C} \rightarrow \overline{\mathscr{U}}_{n} \rightarrow \overline{\mathscr{U}}_{n-1} \rightarrow 0
$$

becomes an exact sequence of filtered logarithmic connections, where $V_{d r}^{\otimes n} \otimes \mathscr{O}_{C}$ has the Hodge filtration induced by the filtration on $V_{d r}^{\otimes n}$.

(iii) The distinguished element $1 \in b^{*} \mathscr{U}_{n}$ belongs to the fibre $b^{*} F^{0} \overline{\mathscr{U}}_{n}$.

We now describe the iterative method of computing the Hodge filtration at level $n$. This method is based on the application presented by Dogra in $[19, \S 4]$. The idea is as follows: we want to compute sub-bundles $F^{\bullet} \mathscr{U}_{n}$ and $F^{\bullet} \mathscr{U}_{n}^{i}$ satisfying Griffiths transversality such that $\left(F^{\bullet} \mathscr{U}_{n}, F^{\bullet} \mathscr{U}_{n}^{i}, G_{n}^{i}\right)$ are the descent datum of sub-bundles $F^{\bullet} \overline{\mathscr{U}}_{n}$ of $\overline{\mathscr{U}}_{n}$ on $C$ satisfying the conditions of Lemma 10. Note that it is clear by a simple induction that we must have $F^{p} \overline{\mathscr{U}}_{n}=0$ for $p>0$ using Definition 23, Remark 24 and Algorithm 4.

The computation of this is contained in the following algorithm.

Algorithm 4 (Computing Hodge filtration on logarithmic universal connection of $C$ with poles along $D$ )

Input:

- Smooth projective curve $C$ over field $K$ of characteristic 0 , a non-empty divisor $D$ defined over $K, X:=C-D$, and a basepoint $b \in X(K)$.

- The logarithmic extension $\overline{\mathscr{U}}_{n}$ of $\mathscr{U}_{n}$ on $X$ computed by Algorithm 1 .

- The Hodge filtration $F^{\bullet} \overline{\mathscr{U}}_{n}$ on $\overline{\mathscr{U}}_{n}$. 


\section{Output:}

- The Hodge filtration $F^{\bullet} \overline{\mathscr{U}}_{n+1}$ on $\overline{\mathscr{U}}_{n}$.

\section{Algorithm:}

(1) For $p>0$ put $F^{p \overline{\mathscr{U}}_{n+1}}=0$.

(2) For $p \leq 0$ do:

(a) Lift generators of $F^{p} \mathscr{U}_{n}$ arbitrarily to sections of $\mathscr{U}_{n+1}$ over $X$ and adjoin the generators of $F^{p} V_{d r}^{\otimes(n+1)} \otimes \mathscr{O}_{C}$.

(b) For all $i$ lift generators of $F^{p} \mathscr{U}_{n}^{i}$ to sections of $\mathscr{U}_{n+1}^{i}$ over $Y_{i}$ and adjoin the generators of $F^{p} V_{d r}^{\otimes(n+1)} \otimes \mathscr{O}_{C}$.

(c) For all $i$ :

(i) Compute the images under $G_{n}^{i}$ of the restrictions to $X \cap Y_{i}$ of the lifts in Step (2)(a).

(ii) Express the images computed in the previous step using restrictions to $X \cap Y_{i}$ of the lifts in Step (2)(b).

(iii) Use the previous step to determine explicit conditions on the lifts from Steps(2)(a) and (b).

(3) For $p=0$ compute generators of $F^{0} \overline{\mathscr{U}}_{n+1}$ such that

(a) The conditions Step (2)(c)(iii) are satisfied.

(b) $1 \in b^{*} F^{0} \overline{\mathscr{U}}_{n}$.

(4) For $p<0$ :

(a) For each $i$ and generator of $F^{p+1} \mathscr{U}_{n+1}^{i}$ compute the image under $\nabla^{i}$ the connection on $\mathscr{U}_{n}^{i}$.

(b) Compute generators of $F^{p} \overline{\mathscr{U}}_{n+1}$ such that:

(i) The conditions of Step (2)(c)(iii) are satisfied.

(ii) $F^{p} \mathscr{U}_{n+1}^{i} \otimes \Omega_{C / K}^{1}(D)$ contains the images computed in Step (4)(a).

Remark 25 Again this algorithm is completely general and it is not immediately obvious that the Algorithm terminates successfully. The obstruction, if it exists, lies in Steps (3) and (4) it is not clear how easy it would be to compute lift satisfying the conditions stated. However, if we are able to do so then Lemma 10 (Hadian's Lemma) ensures that this algorithm will compute the unique Hodge filtration on $\overline{\mathscr{U}}_{n}$. The remainder of this section will be occupied with showing that we can in fact carry out the steps of this algorithm for elliptic curves and odd hyperelliptic curves.

Remark 26 Note that it is easy to see that this algorithm will terminate with the computation of $F^{-n} \overline{\mathscr{U}}_{n}$. Observe that $F^{-n} V_{d r}^{\otimes n}=V_{d r}^{\otimes n}$ and then a simple induction argument shows that $F^{-n} \overline{\mathscr{U}}_{n}=\overline{\mathscr{U}}_{n}$.

Example 2 In this example we compute the Hodge filtration on $\mathscr{U}_{1}$ for $X:=C-D$ as in Definition 9 where $C$ is a smooth projective curve of genus $g$ over $K$ and $D=\left\{d_{1}, \ldots, d_{r}\right\}$ is a divisor defined over $K$. Then $\mathscr{U}_{1}=R_{1} \otimes \mathscr{O}_{X}$ with $R_{1}=\left\langle A_{0}, \ldots, A_{2 g+r-2}\right\rangle_{K}$ where the $A_{k}$ are dual to differentials $\alpha_{k}$. As per Definition 23 note that $F^{0} V_{d r}$ has $K$-basis $\left\{A_{g}, \ldots, A_{2 g+r-2}\right\}$.

Consider the logarithmic extension of $\mathscr{U}_{1}$ to a logarithmic connection $\overline{\mathscr{U}}_{1}$ on $C$ with $\log$ poles along $D$ as computed by Algorithm 1 . This is given by descent datum $\left(\mathscr{U}_{1}^{i}, G_{1}^{i}\right)_{i}$ over an open cover $\left(Y^{i}\right)_{i}$ of $C$, where $Y^{0}=X$. Then we will find that over $X \cap Y^{i}$ 


$$
G_{1}^{i}(1)=1+\sum_{k} h_{k}^{i} A_{k}, G_{1}^{i}\left(A_{l}\right)=A_{l} \text { for all } l
$$

for some $h_{k}^{i} \in K(C)$ such that each $h_{k}^{i}$ is regular on $Y^{i}-D$ and is such that $d h_{k}^{i}-\alpha_{k}$ has logarithmic poles along $D \cap Y_{i}$. Proceeding as in Algorithm 4 we lift the generator 1 of $F^{0} \overline{\mathscr{U}}_{0}$ over each $Y^{i}$ and adjoin the generators of $F^{0} V_{d} r \otimes \mathscr{O}_{C}$, obtaining

$$
1+\sum_{k<g} a_{k}^{i} A_{k}, A_{g}, \ldots, A_{2 g+r-2}
$$

for some $a_{k}^{i} \in H^{0}\left(Y^{i}, \mathscr{O}_{Y^{i}}\right)$. On applying $G_{i}^{1}$ to the lifts (11) over $X$ we find that the generators of $\left.F^{0} \mathscr{U}_{1}^{i}\right|_{X \cap Y^{i}}$ as an $\mathscr{O}_{X \cap Y^{i}}$-module are

$$
1+\sum_{k<g}\left(h_{k}^{i}+a_{k}^{0}\right) A_{k}, A_{g}, \ldots, A_{2 g+r-2}
$$

Restricting the lifts (11) over $Y_{i}$ to $X \cap Y^{i}$ we conclude that

$$
\left.\left(h_{k}^{i}+a_{k}^{0}\right)\right|_{X \cap Y^{i}}=\left.a_{k}^{i}\right|_{X \cap Y^{i}} .
$$

Since $\alpha_{k}$ is regular on $C$ for all $k<g$ then we conclude that each $h_{k}^{i} \in H^{0}\left(Y^{i}, \mathscr{O}_{Y^{i}}\right)$. Therefore, the sections $a_{k}^{0}, a_{k}^{i}-h_{k}^{i}$ glue to give a global section in $H^{0}\left(C, \mathscr{O}_{C}\right) \simeq K$ and so are constant. Since we require that $1 \in b^{*} F^{0} \overline{\mathscr{U}}_{1}$ then we must have that $a_{k}^{0}(b)=0$ and hence that $a_{k}^{0}=0$ for all $k$. Therefore, the Hodge filtration at on $F^{0} \overline{\mathscr{U}}_{1}$ is generated over $X$ by

$$
1, A_{g}, \ldots, A_{2 g+r-2} \text {. }
$$

\subsection{A constructive algorithm for affine elliptic and hyperelliptic curves}

In what follows we present a proof that the extensions $\overline{\mathscr{U}}$ we computed by Algorithm 3 in Section 3 have a unique Hodge filtration and, furthermore, we show that there are explicit conditions which uniquely determine the generators of the filtration at each level. Finally, we present an algorithm which may be used to compute the Hodge filtration iteratively.

Before stating the main theorem of this section we first identify an ordered $K$-basis of $F^{0} V_{d r}^{\otimes n}$ with respect to the basis in words of length $n$ with the graded lexicographic ordering of Sect. 3.3. Recall that in Definition 23 we identified this space as being the $K$-span of the set of words of length $n$ in $A_{g}, \ldots, A_{2 g-1}$.

Lemma 11 Let $\mathscr{F}_{n}$ be the set

$$
\left\{1+\sum_{i=0}^{n-1} f_{i}(2 g)^{i}: f_{i} \in\{g, \ldots, 2 g-1\}\right\} .
$$

Then $w_{n}^{f}$ for $f \in \mathscr{F}_{n}$ forms a $K$-basis for $F^{0} V_{d r}^{\otimes n}$.

Proof By definition $F^{0} V_{d r}$ has $K$-basis $\left\{A_{g}, \ldots, A_{2 g-1}\right\}=\left\{w_{1}^{1+g}, \ldots, w_{1}^{1+2 g-1}\right\}$. Hence the statement of the lemma is true in the case that $n=1$. We now proceed by induction. Suppose that the statement of the lemma is true for some $n$. Then $F^{0} V_{d r}^{\otimes n}$ has $K$-basis $\left\{w_{n}^{f} \mid f \in \mathscr{F}_{n}\right\}$ where $\mathscr{F}_{n}$ is as in the statement of the lemma. 
Note that $F^{0} V_{d r}^{\otimes n+1}$ has $K$-basis $\bigcup_{i=g}^{2 g-1}\left\{A_{i} w_{n}^{f} \mid f \in \mathscr{F}_{n}\right\}=\bigcup_{i=g}^{2 g-1}\left\{w_{n+1}^{(2 g)^{n} i+f} \mid f \in \mathscr{F}_{n}\right\}$ upon recalling that $A_{i} w_{l}^{k}=w_{l+1}^{k+(2 g)^{l} i}$. Therefore, we conclude that the statement of the lemma is true for $n+1$. Hence, by induction we deduce that the lemma is true for all $n \geq 1$.

Remark 27 For the sake of brevity in the proof of Theorem 4 below we define $\mathscr{F}_{0}:=\{1\}$ since $F^{0} \overline{\mathscr{U}}_{0}=F^{0} \mathscr{O}_{C}=\mathscr{O}_{C}$.

The main theorem of this section may now be stated as follows.

Theorem 4 Let $C$ be an elliptic curve or an odd hyperelliptic curve of genus $g$ over a field $K$ of characteristic 0 and let $\infty$ be the point at infinity. Let $X:=C-\{\infty\}$ be the affine curve with model $y^{2}=f(x)$ for some $f(x) \in K[x]$ where $\operatorname{deg} f=2 g+1$. Let $b \in X(K)$ be $a$ basepoint. Let $\mathscr{U}=\left\{\left(\mathscr{U}_{n}, 1\right)\right\}_{n \geq 0}$ be the universal connection on $X$ as in Definition 9. Let $\overline{\mathscr{U}}=\left\{\left(\overline{\mathscr{U}}_{n}, 1\right)\right\}$ be the logarithmic extension of $\mathscr{U}$ computed by Algorithm 3.

Then there is a unique filtration $F^{\bullet} \overline{\mathscr{U}}_{n}$ of $\overline{\mathscr{U}}_{n}$ for all $n$ and this filtration $F^{\bullet} \overline{\mathscr{U}}_{n}$ is explicitly computable; the logarithmic connection $\overline{\mathscr{U}}_{n}$ has the structure of a filtered logarithmic connection on $C$ fitting into an exact sequence

$$
0 \rightarrow V_{d r}^{\otimes n} \otimes \mathscr{O}_{C} \rightarrow \overline{\mathscr{U}}_{n} \rightarrow \overline{\mathscr{U}}_{n-1} \rightarrow 0
$$

of filtered logarithmic connections; and $1 \in b^{*} F^{0} \overline{\mathscr{U}}_{n}$ for all $n$.

Since $F^{1} \overline{\mathscr{U}}_{0}=F^{1} \mathscr{O}_{C}=0$ and $F^{1} V_{d r}^{\otimes n}=0$ we easily deduce by induction that $F^{1} \overline{\mathscr{U}}_{n}=$ 0 for all $n$. So the first non-trivial case we consider is the computation of the $F^{0}$ part of the filtration. The proof can be conveniently split up into the following three parts. First, we derive necessary and sufficient conditions on lifts of generators of $F^{0} \overline{\mathscr{U}}_{n}$ over $X$ and $Y$ such that any lifts satisfying them will generate a sub-bundle of $\overline{\mathscr{U}}_{n}$ satisfying the conditions of Lemma 10. Second, we show that any lift satisfying these conditions are unique. Finally, we provide an algorithm to compute such lifts, demonstrating that they exist.

In what follows we shall need to make use of the following function.

Definition 24 Let $(i, j, p, q) \in \mathbb{Z}^{4}$. Then define

$$
\tau(i, j, p, q):= \begin{cases}1 & \text { if } i=j+(\psi(i, 0, p, q)-1) q^{p} \\ 0 & \text { otherwise }\end{cases}
$$

where $\psi$ is the function in Definition 20.

Lemma 12 Suppose that the conditions in the statement of Theorem 4 are satisfied. Then suppose that

$\left(A_{n}\right) F^{0} \mathscr{U}_{n}$ is generated as an $\mathscr{O}_{X}$-module by

$$
\begin{gathered}
w_{n}^{f} \quad \text { where } f \in \mathscr{F}_{n} \\
w_{m}^{f}+\sum_{l=m+1}^{n} \sum_{k=1}^{(2 g)^{l}} a_{m, f}^{l, k} w_{l}^{k} \quad \text { where } m \in\{0, \ldots, n-1\}, f \in \mathscr{F}_{m}
\end{gathered}
$$

for some $a_{m, f}^{l, k} \in H^{0}\left(X, \mathscr{O}_{X}\right)$ such that $a_{m, f}^{l, f^{\prime}}=0$ for all $f^{\prime} \in \mathscr{F}_{l}$ and $a_{0,1}^{l, k}(b)=0$ for all $l, k$. 
$\left(B_{n}\right) F^{0} \mathscr{U}_{n}^{\prime}$ is generated as an $\mathscr{O}_{Y}$-module by

$$
\begin{gathered}
w_{n}^{f} \quad \text { where } f \in \mathscr{F}_{n} \\
w_{m}^{f}+\sum_{l=m+1}^{n} \sum_{k=1}^{(2 g)^{l}} b_{m, f}^{l, k} w_{l}^{k} \quad \text { where } m \in\{0, \ldots, n-1\}, f \in \mathscr{F}_{m}
\end{gathered}
$$

for some $b_{m, f}^{l, k} \in H^{0}\left(Y, \mathscr{O}_{Y}\right)$ such that $b_{m, f}^{l, f^{\prime}}=0$ for all $f^{\prime} \in \mathscr{F}_{l}$.

Suppose there are lifts $\left(A_{n+1}\right)$ and $\left(B_{n+1}\right)$ generating a sub-bundle $F^{0} \overline{\mathscr{U}}_{n+1}$ of $\overline{\mathscr{U}}_{n+1}$ such that the following sequence is exact

$$
0 \rightarrow F^{0} V_{d r}^{\otimes(n+1)} \otimes \mathscr{O}_{C} \rightarrow F^{0} \overline{\mathscr{U}}_{n+1} \rightarrow F^{0} \overline{\mathscr{U}}_{n} \rightarrow 0 .
$$

Then the lifts satisfy the following conditions on restriction to $X \cap Y$ :

$I_{n}:$ For $f \in \mathscr{F}_{n}$ and $k \in\left\{1, \ldots,(2 g)^{n+1}\right\}$

$$
\begin{aligned}
b_{n, f}^{n+1, k}= & a_{n, f}^{n+1, k}+h_{n+1}^{\psi(k, 0, n, 2 g), n} \tau(k, f, n, 2 g) \\
& -\sum_{f^{\prime} \in \mathscr{F}_{n+1}} h_{n+1}^{\psi\left(f^{\prime}, 0, n, 2 g\right), n} \tau\left(f^{\prime}, f, m, 2 g\right) \delta_{f^{\prime}, k}
\end{aligned}
$$

$I_{m}:$ For $f \in \mathscr{F}_{m}$ and $k \in\left\{1, \ldots,(2 g)^{n+1}\right\}$

$$
\begin{aligned}
b_{m, f}^{n+1, k}= & a_{m, f}^{n+1, k}+h_{n+1}^{\psi(k, 0, m, 2 g), m} \tau(k, f, m, 2 g) \\
& -\sum_{f^{\prime} \in \mathscr{F}_{n+1}} h_{n+1}^{\psi\left(f^{\prime}, 0, m, 2 g\right), m} \tau\left(f^{\prime}, f, m, 2 g\right) \delta_{f^{\prime}, k} \\
& +\sum_{l=m+1}^{n} a_{m, f}^{l, k-(\psi(k, 0, l, 2 g)-1)(2 g)^{l}} h_{n+1}^{\psi(k, 0, l, 2 g), l} \\
& -\sum_{p=m+1}^{n} \sum_{f^{\prime} \in \mathscr{F}_{p}} b_{p, f^{\prime}}^{n+1, k} h_{p}^{\psi\left(f^{\prime}, 0, m, 2 g\right), m} \tau\left(f^{\prime}, f, m, 2 g\right)
\end{aligned}
$$

where $m \in\{0, \ldots, n-1\}$. Additionally, $a_{0,1}^{n+1, k}(b)=0$ for all $k$.

Proof Assume that $\left(A_{n}\right)$ and $\left(B_{n}\right)$ hold for some $n$. We require that $F^{0} \overline{\mathscr{U}}_{n+1}$ fits into an exact sequence

$$
0 \rightarrow F^{0} V_{d r}^{\otimes(n+1)} \otimes \mathscr{O}_{C} \rightarrow F^{0} \overline{\mathscr{U}}_{n+1} \rightarrow F^{0} \overline{\mathscr{U}}_{n} \rightarrow 0 .
$$

Considering this exact sequence over $X$ we lift the generators of $F^{0} \mathscr{U}_{n}$ arbitrarily to $F^{0} \mathscr{U}_{n+1}$ and suppose said lifts, together with the generators of $F^{0} V_{d r}^{\otimes(n+1)} \otimes \mathscr{O}_{C}$, are generators of $F^{0} \mathscr{U}_{n+1}$. We let these generators be

$$
\begin{gathered}
T_{n+1}^{f}:=w_{n+1}^{f} \quad \text { where } f \in \mathscr{F}_{n+1} \\
T_{m}^{f}:=w_{m}^{f}+\sum_{l=m+1}^{n+1} \sum_{k=1}^{(2 g)^{l}} a_{m, f}^{l, k} w_{l}^{k} \quad \text { where } m \in\{0, \ldots, n\}, f \in \mathscr{F}_{m}
\end{gathered}
$$

for some $a_{m, f}^{n+1, k} \in H^{0}\left(X, \mathscr{O}_{X}\right)$. Since we suppose these are generators of $F^{0} \mathscr{U}_{n+1}$ we may assume that $a_{m, f}^{n+1, f^{\prime}}=0$ for all $f^{\prime} \in \mathscr{F}_{n+1}$. 
We repeat this over $Y$ obtaining lifts

$$
\begin{gathered}
S_{n+1}^{f}:=w_{n+1}^{f} \quad \text { where } f \in \mathscr{F}_{n+1} \\
S_{m}^{f}:=w_{m}^{f}+\sum_{l=m+1}^{n+1} \sum_{k=1}^{(2 g)^{l}} b_{m, f}^{l, k} w_{l}^{k} \quad \text { where } m \in\{0, \ldots, n\}, f \in \mathscr{F}_{m}
\end{gathered}
$$

for some $b_{m, f}^{n+1, k} \in H^{0}\left(X, \mathscr{O}_{X}\right)$. We take these as candidate generators for $F^{0} \mathscr{U}_{n+1}^{\prime}$, and again we may assume that $b_{m, f}^{n+1, f^{\prime}}=0$ for all $f^{\prime} \in \mathscr{F}_{n+1}$.

We need to show the following that over $X \cap Y$ we have an isomorphism of bundles

$$
G_{n+1}:\left.\left.F^{0} \mathscr{U}_{n+1}\right|_{X \cap Y} \cong F^{0} \mathscr{U}_{n+1}^{\prime}\right|_{X \cap Y} .
$$

We consider the action of $G_{n+1}$ on the candidate generators for $F^{0} \mathscr{U}_{n+1}$. In what follows we must work over $X \cap Y$, but we suppress the notation $\left.\right|_{X \cap Y}$ for ease of exposition. Using Lemma 9 we determine the action of $G_{n+1}$ on the candidate generators $T_{m}^{f}$ of $F^{0} \mathscr{U}_{n+1}$. We summarise these calculations below:

$$
\begin{aligned}
T_{n+1}^{f} \mapsto \tilde{T}_{n+1}^{f} & :=w_{n+1}^{f} \\
T_{m}^{f} \mapsto \tilde{T}_{m}^{f}:= & w_{m}^{f}+\sum_{s=m+1}^{n+1} \sum_{t=1}^{(2 g)^{s-m}} w_{s}^{f+(t-1)(2 g)^{m}} h_{s}^{t, m} \\
& +\left(1-\delta_{m, n}\right) \sum_{l=m+1}^{n} \sum_{k=1}^{(2 g)^{l}} a_{m, f}^{l, k}\left(w_{l}^{k}+\sum_{s=l+1}^{n+1} \sum_{t=1}^{(2 g)^{s-l}} w_{s}^{k+(t-1)(2 g)^{l}} h_{s}^{t, l}\right) \\
& +\sum_{k=1}^{(2 g)^{n+1}} a_{m, f}^{n+1, k} w_{n+1}^{k} .
\end{aligned}
$$

for $m \in\{0, \ldots, n\}$ and $f \in \mathscr{F}_{m}$. Define $\tilde{S}_{m}^{f}$ recursively as follows

$$
\begin{aligned}
\tilde{S}_{n+1}^{f} & :=S_{n+1}^{f} \\
\tilde{S}_{m}^{f} & :=\tilde{T}_{m}^{f}-\sum_{p=m+1}^{n+1} \sum_{f^{\prime} \in \mathscr{F}_{p}} \lambda_{p}^{f^{\prime}} \tilde{S}_{p}^{f^{\prime}}
\end{aligned}
$$

where $m \in\{0, \ldots, n\}$ and $\lambda_{p}^{f^{\prime}} \in H^{0}\left(X \cap Y, \mathscr{O}_{X \cap Y}\right)$ is the co-efficient of $w_{p}^{f^{\prime}}$ appearing in $\tilde{T}_{m}^{f}$. As the coefficient of $w_{m^{\prime}}^{f^{\prime}}$ in $\tilde{S}_{m}^{f}$ is 1 if $f=f^{\prime}, m=m^{\prime}$ and is 0 otherwise we have the following equality for all $m, f$

$$
\tilde{S}_{m}^{f}=\left.S_{m}^{f}\right|_{X \cap Y} .
$$

Using Lemma 11 we calculate that $\lambda_{p}^{f^{\prime}}=h_{p}^{\psi\left(f^{\prime}, 0, m, 2 g\right), m} \tau\left(f^{\prime}, f, m, 2 g\right)$. The equality (12) is trivially true by definition when $m=n+1$. For $m<n+1$ we proceed recursively, finding first that

$$
\begin{aligned}
\tilde{S}_{n}^{f}= & w_{n}^{f}+\sum_{t=1}^{2 g} w_{n+1}^{f+(t-1)(2 g)^{n}} h_{n+1}^{t, n}+\sum_{k=1}^{(2 g)^{n+1}} a_{n, f}^{n+1, k} w_{n+1}^{k} \\
& -\sum_{f^{\prime} \in \mathscr{F}_{n+1}} h_{n+1}^{\psi\left(f^{\prime}, 0, n, 2 g\right), n} \tau\left(f^{\prime}, f, n, 2 g\right) w_{n+1}^{f^{\prime}}=w_{n}^{f}+\sum_{k=1}^{(2 g)^{n+1}} b_{n, f}^{n+1, k} w_{n+1}^{k} .
\end{aligned}
$$


By considering the coefficient of $w_{n+1}^{q}$ on both sides of the equality above we conclude that on restriction to $X \cap Y$ the sections $a_{n, f}^{n+1, q}, b_{n, f}^{n+1, q}$ must satisfy

$b_{n, f}^{n+1, q}=a_{n, f}^{n+1, q}+h_{n+1}^{\psi(q, 0, n, 2 g), n} \tau(q, f, m, 2 g)-\sum_{f^{\prime} \in \mathscr{F}_{n+1}} h_{n+1}^{\psi\left(f^{\prime}, 0, n, 2 g\right), n} \tau\left(f^{\prime}, f, m, 2 g\right) \delta_{f^{\prime}, q}$

Now suppose that $m \in\{0, \ldots, n-1\}$. Then if we suppose that we have an equality $\tilde{S}_{p}^{f}=\left.S_{p}^{f}\right|_{X \cap Y}$ for $p>m$ we find that

$$
\begin{aligned}
\tilde{S}_{m}^{f}= & w_{m}^{f}+\sum_{s=m+1}^{n+1} \sum_{t=1}^{(2 g)^{s-m}} w_{s}^{f+(t-1)(2 g)^{m}} h_{s}^{t, m}+\sum_{k=1}^{(2 g)^{n+1}} a_{m, f}^{n+1, k} w_{n+1}^{k} \\
& +\sum_{l=m+1}^{n} \sum_{k=1}^{(2 g)^{l}} a_{m, f}^{l, k}\left(w_{l}^{k}+\sum_{s=l+1}^{n+1} \sum_{t=1}^{(2 g)^{s-l}} w_{s}^{k+(t-1)(2 g)^{l}} h_{s}^{t, l}\right) \\
& -\sum_{p=m+1}^{n} \sum_{f^{\prime} \in \mathscr{F}_{p}} h_{p}^{\psi\left(f^{\prime}, 0, m, 2 g\right), m} \tau\left(f^{\prime}, f, m, 2 g\right)\left(w_{p}^{f^{\prime}}+\sum_{l=p+1}^{n+1} \sum_{k=1}^{(2 g)^{l}} b_{p, f^{\prime}}^{l, k} w_{l}^{k}\right) \\
& -\sum_{f^{\prime} \in \mathscr{F}} h_{n+1}^{\psi\left(f^{\prime}, 0, m, 2 g\right), m} \tau\left(f^{\prime}, f, m, 2 g\right) w_{n+1}^{f^{\prime}} \\
= & w_{m}^{f}+\sum_{l=m+1}^{n+1} \sum_{k=1}^{(2 g)^{l}} b_{m, f}^{l, k} w_{l}^{k}=S_{m}^{f}
\end{aligned}
$$

Since the $a_{m, f}^{l, q}, b_{m, f}^{l, q}$ are known for $l<n+1$, we may assume that we have equality of coefficients among words of length at most $n$. So we need only concern ourselves with words of length $n+1$ in the above. We consider the coefficient of the word $w_{n+1}^{q}$ where $q \in\left\{1, \ldots,(2 g)^{n+1}\right\}$. Arguing as when $m=n$ we conclude that on restriction to $X \cap Y$ the sections $a_{m, f}^{n+1, q}, b_{m, f}^{n+1, q}$ satisfy

$$
\begin{aligned}
b_{m, f}^{n+1, q}= & a_{m, f}^{n+1, q}+h_{n+1}^{\psi(q, 0, m, 2 g), n} \tau(q, f, m, 2 g) \\
& -\sum_{f^{\prime} \in \mathscr{F}_{n+1}} h_{n+1}^{\psi\left(f^{\prime}, 0, m, 2 g\right), m} \tau\left(f^{\prime}, f, m, 2 g\right) \delta_{f^{\prime}, q} \\
& +\sum_{l=m+1}^{n} a_{m, f}^{l, q-(\psi(q, 0, l, 2 g)-1)(2 g)^{l}} h_{n+1}^{\psi(q, 0, l, 2 g), l} \\
& -\sum_{p=m+1}^{n} \sum_{f^{\prime} \in \mathscr{F}_{p}} b_{p, f^{\prime}}^{n+1, q} h_{p}^{\psi\left(f^{\prime}, 0, m, 2 g\right), m} \tau\left(f^{\prime}, f, m, 2 g\right) .
\end{aligned}
$$

Therefore, we have shown that any such lifts must satisfy the conditions in the statement of the lemma. 
We now show that any sections satisfying these conditions must be unique.

Lemma 13 Suppose that there are lifts $S_{m}^{f}, T_{m}^{f}$ for $m \in\{0, \ldots, n\}$ and $f \in \mathscr{F}_{m}$ satisfying the conditions $\left(I_{m}\right)$ of Lemma 12. Then the lifts $S_{m}^{f}, T_{m}^{f}$ are unique.

Proof We suppose that we have a second set of lifts satisfying the conditions $I_{m}$ of Lemma 12:

$$
\begin{aligned}
\bar{T}_{m}^{f} & :=w_{m}^{f}+\sum_{l=m+1}^{n} \sum_{k=1}^{(2 g)^{l}} a_{m, f}^{l, k} w_{l}^{k}+\sum_{k=1}^{(2 g)^{n+1}} \tilde{a}_{m, f}^{n+1, k} w_{n+1}^{k} \\
\bar{S}_{m}^{f} & :=w_{m}^{f}+\sum_{l=m+1}^{n} \sum_{k=1}^{(2 g)^{l}} b_{m, f}^{l, k} w_{l}^{k}+\sum_{k=1}^{(2 g)^{n+1}} \tilde{b}_{m, f}^{n+1, k} w_{n+1}^{k}
\end{aligned}
$$

where $m \in\{0, \ldots, n\}, f \in \mathscr{F}_{m}$ and $\tilde{a}_{m, f}^{n+1, k}, \tilde{b}_{m, f}^{n+1, k}$ are sections in $H^{0}\left(X, \mathscr{O}_{X}\right)$ and $H^{0}\left(Y, \mathscr{O}_{Y}\right)$ respectively. First, for $f \in \mathscr{F}_{n}$ and $k \in\left\{1, \ldots,(2 g)^{n+1}\right\}$ we consider the difference

$$
b_{n, f}^{n+1, k}-\tilde{b}_{n, f}^{n+1, k}=a_{n, f}^{n+1, k}-\tilde{a}_{n, f}^{n+1, k} .
$$

Note that since $a_{n, f}^{n+1, k}-\tilde{a}_{n, f}^{n+1, k} \in H^{0}\left(X, \mathscr{O}_{X}\right)$ and $b_{n, f}^{n+1, k}-\tilde{b}_{n, f}^{n+1, k} \in H^{0}\left(Y, \mathscr{O}_{Y}\right)$ and these sections agree on $X \cap Y$ they must glue to give a global section in $H^{0}\left(C, \mathscr{O}_{C}\right)$. Therefore, $a_{n, f}^{n+1, k}-\tilde{a}_{n, f}^{n+1, k}$ and $b_{n, f}^{n+1, k}-\tilde{b}_{n, f}^{n+1, k}$ are constant and equal.

We now proceed by induction on $m \in\{0, \ldots, n-1\}$ with the following induction hypothesis:

$\left(C_{m}\right)$ : For all $f \in \mathscr{F}_{m}, f^{\prime} \in \mathscr{F}_{m+1}$ and $k, k^{\prime} \in\left\{1, \ldots,(2 g)^{n+1}\right\}$ the following hold:

1. $b_{m, f}^{n+1, k}-\tilde{b}_{m, f}^{n+1, k}=a_{m, f}^{n+1, k}-\tilde{a}_{m, f}^{n+1, k} \in H^{0}\left(C, \mathscr{O}_{C}\right)=K$

2. $0=b_{m+1, f^{\prime}}^{n+1, k^{\prime}}-\tilde{b}_{m+1, f^{\prime}}^{n+1, k^{\prime}}=a_{m+1, f^{\prime}}^{n+1, k^{\prime}}-\tilde{a}_{m+1, f^{\prime}}^{n+1, k^{\prime}} \in H^{0}\left(C, \mathscr{O}_{C}\right)=K$

Take $m=n-1$ as our base case. To show $\left(C_{n-1}\right)$ we consider for each $f \in \mathscr{F}_{n-1}$ and $k \in\left\{1, \ldots,(2 g)^{n+1}\right\}$ the difference

$$
\begin{aligned}
& b_{n-1, f}^{n+1, k}-\tilde{b}_{n-1, f}^{n+1, k}=a_{n-1, f}^{n+1, k}-\tilde{a}_{n-1, f}^{n+1, k} \\
& -\sum_{f^{\prime} \in \mathscr{F}_{n}}\left(b_{n, f^{\prime}}^{n+1, k}-\tilde{b}_{n, f^{\prime}}^{n+1, k}\right) h_{n}^{\psi\left(f^{\prime}, 0, n-1,2 g\right), n-1} \tau\left(f^{\prime}, f, n-1,2 g\right) .
\end{aligned}
$$

Recall that $h_{n}^{\psi\left(f^{\prime}, 0, n-1,2 g\right), n-1}=h_{1}^{\psi\left(f^{\prime}, 0, n-1,2 g\right), 0}$. As $f^{\prime} \in \mathscr{F}_{n}$ we find that $g+1 \leq$ $\psi\left(f^{\prime}, 0, n-1,2 g\right) \leq 2 g$ for each $f^{\prime} \in \mathscr{F}_{n}$. Using Algorithm 3 we note that $\alpha_{t-1}$ has a pole of order $2(t-g)$ at $\infty$ and $h_{1}^{t, 0}$ is chosen so that $d h_{1}^{t, 0}-\alpha_{t-1}$ has at worst logarithmic poles at $\infty$ and hence $h_{1}^{t, 0}$ has a pole of order $2(t-g)-1$ at $\infty$ for $t>g$. Hence the order of the pole of $h_{n}^{\psi\left(f^{\prime}, 0, n-1,2 g\right), n-1}$ at $\infty$ is in $\{1,3, \ldots, 2 g-1\}$.

Note that $x$ has a pole of order 2 at $\infty$ and $y$ has a pole of order $2 g+1$ at $\infty$ and hence $a_{n-1, f}^{n+1, k}-\tilde{a}_{n-1, f}^{n+1, k}$ can only have a pole of order lying in $\{2,4, \ldots, 2 g\} \cup\{o \in \mathbb{Z}: o \geq 2 g+1\}$. Since $b_{n-1, f}^{n+1, k}-\tilde{b}_{n-1, f}^{n+1, k}$ is regular at $\infty$ the only way we can have equality over $X \cap Y$ as in (13) is if

$$
\left(b_{n, f^{\prime}}^{n+1, k}-\tilde{b}_{n, f^{\prime}}^{n+1, k}\right) \tau\left(f^{\prime}, f, n-1,2 g\right)=0
$$


for all $f^{\prime} \in \mathscr{F}_{n}$ and $k \in\left\{1, \ldots,(2 g)^{n+1}\right\}$. By varying over all $f \in \mathscr{F}_{n-1}$ we deduce, therefore, that

$$
b_{n, f^{\prime}}^{n+1, k}-\tilde{b}_{n, f^{\prime}}^{n+1, k}=a_{n, f^{\prime}}^{n+1, k}-\tilde{a}_{n, f^{\prime}}^{n+1, k}=0
$$

for all $f^{\prime} \in \mathscr{F}_{n}$ and $k \in\left\{1, \ldots,(2 g)^{n+1}\right\}$. Finally, this implies that

$$
b_{n-1, f}^{n+1, k}-\tilde{b}_{n-1, f}^{n+1, k}=a_{n-1, f}^{n+1, k}-\tilde{a}_{n-1, f}^{n+1, k}
$$

and as before we conclude that these sections glue to a global section and hence are constant. Therefore, we find that $\left(C_{n-1}\right)$ holds. By considering the differences $b_{m, f}^{n+1, k}-\tilde{b}_{m, f}^{n+1, k}$ for $f \in \mathscr{F}_{m}$ and $k \in\left\{1, \ldots,(2 g)^{n+1}\right\}$ we conclude that $\left(C_{m}\right)$ holds for all $m$.

Therefore, for $m \geq 1$ the sections $a_{m, f}^{n+1, k}, b_{m, f}^{n+1, k}$ are unique. Additionally, $b_{0, f}^{n+1, k}-$ $\tilde{b}_{0, f}^{n+1, k}=a_{0, f}^{n+1, k}-\tilde{a}_{0, f}^{n+1, k} \in K$. However, recall that our lifts should be such that $1 \in$ $b^{*} F^{0} \overline{\mathscr{U}}_{n+1}$. Therefore, these sections must be 0 at $b$ and hence they must be exactly 0 . Hence, for $m \geq 0$ the sections $a_{m, f}^{n+1, k}, b_{m, f}^{n+1, k}$ are unique and thus the lifts are unique.

The existence of suitable lifts is demonstrated by the following Algorithm.

Algorithm 5 (Computing $F^{0} \overline{\mathscr{U}}_{n}$ on elliptic and odd hyperelliptic curves $X=C-\{\infty\}$ )

\section{Input}

- $C$ a complete elliptic or odd hyperelliptic curve of genus $g$ over a field $K, D=\{\infty\}$ and $X:=C-D$ with affine model $y^{2}=f(x)$ for some $f(x) \in K[x]$ with $\operatorname{deg} f=2 g+1$.

- The logarithmic extension $\overline{\mathscr{U}}_{n}$ of $\mathscr{U}_{n}$ computed by Algorithm 5 .

- Generators of $F^{0} \mathscr{U}_{n}$ as an $\mathscr{O}_{X}$-module:

$$
\begin{aligned}
w_{n}^{f} \quad \text { where } f \in \mathscr{F}_{n} \\
w_{m}^{f}+\sum_{l=m+1}^{n} \sum_{k=1}^{(2 g)^{l}} a_{m, f}^{l, k} w_{l}^{k} \quad \text { where } m \in\{0, \ldots, n-1\}, f \in \mathscr{F}_{m}
\end{aligned}
$$

and generators of $F^{0} \mathscr{U}_{n}^{\prime}$ as an $\mathscr{O}_{Y}$-module:

$$
\begin{gathered}
w_{n}^{f} \quad \text { where } f \in \mathscr{F}_{n} \\
w_{m}^{f}+\sum_{l=m+1}^{n} \sum_{k=1}^{(2 g)^{l}} b_{m, f}^{l, k} w_{l}^{k} \quad \text { where } m \in\{0, \ldots, n-1\}, f \in \mathscr{F}_{m} .
\end{gathered}
$$

\section{Output}

- Generators of $F^{0} \mathscr{U}_{n+1}$ as an $\mathscr{O}_{X}$-module:

$$
\begin{gathered}
w_{n}^{f} \quad \text { where } f \in \mathscr{F}_{n} \\
w_{m}^{f}+\sum_{l=m+1}^{n+1} \sum_{k=1}^{(2 g)^{l}} a_{m, f}^{l, k} w_{l}^{k} \quad \text { where } m \in\{0, \ldots, n-1\}, f \in \mathscr{F}_{m}
\end{gathered}
$$

and generators of $F^{0} \mathscr{U}_{n+1}^{\prime}$ as an $\mathscr{O}_{Y}$-module:

$$
\begin{gathered}
w_{n+1}^{f} \quad \text { where } f \in \mathscr{F}_{n+1} \\
w_{m}^{f}+\sum_{l=m+1}^{n+1} \sum_{k=1}^{(2 g)^{l}} b_{m, f}^{l, k} w_{l}^{k} \quad \text { where } m \in\{0, \ldots, n\}, f \in \mathscr{F}_{m} .
\end{gathered}
$$




\section{Algorithm}

(1) Fix a local parameter $\pi$ at $\infty$ e.g. $\pi=\frac{x^{g}}{y}$.

(2) Compute the $\pi$-expansion of the $\mathscr{O}_{X}$-sections $x$ and $y$ and each $h_{1}^{t, 0}$ :

$$
\begin{aligned}
x(\pi) & =\chi \pi^{-2}+\cdots \\
y(\pi) & =\gamma \pi^{-(2 g+1)}+\cdots \\
h_{1}^{t, 0}(\pi) & =\eta_{1}^{t, 0} \pi^{-(2(t-g)-1)}+\cdots
\end{aligned}
$$

for some constants $\chi, \gamma, \eta_{1}^{t, 0} \in K$.

(3) For $f \in \mathscr{F}_{n+1}$ do:

$$
\begin{aligned}
& T_{n+1}^{f}:=w_{n+1}^{f} \\
& S_{n+1}^{f}:=w_{n+1}^{f}
\end{aligned}
$$

(4) For $f \in \mathscr{F}_{n}$ do:

(a) For $k \in \mathscr{F}_{n+1}$ do:

$$
a_{n, f}^{n+1, k}, b_{n, f}^{n+1, k}:=0
$$

(b) For $k \in\left\{1, \ldots,(2 g)^{n+1}\right\}-\mathscr{F}_{n+1}$ do:

$$
A_{n, f}^{n+1, k}:=0
$$

(c) Let $m:=n-1$.

(5) While $m \geq 0$, for $f \in \mathscr{F}_{m}$ and $k \in\left\{1, \ldots,(2 g)^{n+1}\right\}$ do:

If: $k \in \mathscr{F}_{n+1}$ do:

$$
a_{m, f}^{n+1, k}, b_{m, f}^{n+1, k}:=0
$$

Else:

(a) Define

$$
\begin{aligned}
s_{m, f}^{n+1, k}:= & h_{n+1}^{\psi(k, 0, m, 2 g), m} \tau(k, f, m, 2 g)+\sum_{l=m+1}^{n} a_{m, f}^{l, k-(\psi(k, 0, l, 2 g)-1)(2 g)^{l}} h_{n+1}^{\psi(k, 0, l, 2 g), l} \\
& -\sum_{f^{\prime} \in \mathscr{F}_{m+1}}\left(b_{m+1, f^{\prime}}^{n+1, k}-\lambda_{m+1, f^{\prime}}^{n+1, k}\right) h_{m+1}^{\psi\left(f^{\prime}, 0, m, 2 g\right), m} \tau\left(f^{\prime}, f, m, 2 g\right) \\
& -\left(1-\delta_{m, n-1}\right) \sum_{p=m+2}^{n} \sum_{f^{\prime} \in \mathscr{F}_{p}} b_{p, f^{\prime}}^{n+1, k} h_{p}^{\psi\left(f^{\prime}, 0, m, 2 g\right), m} \tau\left(f^{\prime}, f, m, 2 g\right) .
\end{aligned}
$$

(b) Compute the $\pi$-expansion of $s_{m, f}^{n+1, k}$.

(c) Compute $A_{m, f}^{n+1, k}(x, y) \in K[x, y] /\left(y^{2}-f(x)\right)$ such that

$$
A_{m, f}^{n+1, k}(x(\pi), y(\pi))+s_{m, f}^{n+1, k}(\pi) \equiv 0 \quad \bmod \pi^{-(2 g-1)} K[[\pi]] .
$$


(d) Suppose that

$$
A_{m, f}^{n+1, k}(x(\pi), y(\pi))+s_{m, f}^{n+1, k}(\pi)=\mu_{2 g-1} \pi^{-(2 g-1)}+\cdots
$$

Then define $\lambda_{m+1, f+(2 g-1)(2 g)^{m}}^{n+1, k}:=\frac{\mu_{2 g-1}}{\eta_{1}^{2 g, 0}}$.

(e) Define

$$
a_{m+1, f+(2 g-1)(2 g)^{m}}^{n+1, k}:=A_{m+1, f+(2 g-1)(2 g)^{m}}^{n+1, k}+\lambda_{m+1, f+(2 g-1)(2 g)^{m}}^{n+1, k} .
$$

(f) Suppose that

$$
\begin{aligned}
& A_{m, f}^{n+1, k}(x(\pi), y(\pi))+s_{m, f}^{n+1, k}(\pi)-\lambda_{m+1, f+(2 g-1)(2 g)^{m}}^{n+1, k} h_{m+1}^{2 g, m}(\pi) \\
& \quad=\mu_{2 g-2} \pi^{-(2 g-2)}+\cdots
\end{aligned}
$$

Then define $A_{m, f}^{n+1, k}:=A_{m, f}^{n+1, k}(x, y)-\frac{\mu_{2 g-2}}{\chi^{g-1}} x^{g-1}$.

(g) Let $\tilde{t}=2 g-1$. While $\tilde{t} \neq g+1$ do

(i) Suppose that

$$
\begin{aligned}
& A_{m, f}^{n+1, k}(x(\pi), y(\pi))+s_{m, f}^{n+1, k}(\pi)-\sum_{t=\tilde{t}+1}^{2 g} \lambda_{m+1, f+(t-1)(2 g)^{m}}^{n+1, k} h_{m+1}^{t, m}(\pi) \\
& \quad=\mu_{2(\tilde{t}-g)-1} \pi^{-(2(\tilde{t}-g)-1)}+\cdots
\end{aligned}
$$

Then define $\lambda_{m+1, f+(\tilde{t}-1)(2 g)^{m}}^{n+1, k}:=\frac{\mu_{2(\tilde{t}-g)-1}}{\eta_{m+1}^{\tilde{t}, m}}$.

(ii) Define

$$
a_{m+1, f+(\tilde{t}-1)(2 g)^{m}}^{n+1, k}:=A_{m+1, f+(\tilde{t}-1)(2 g)^{m}}^{n+1, k}+\lambda_{m+1, f+(\tilde{t}-1)(2 g)^{m}}^{n+1, k} .
$$

(iii) Suppose that

$$
\begin{aligned}
& A_{m, f}^{n+1, k}(x(\pi), y(\pi))+s_{m, f}^{n+1, k}(\pi)-\sum_{t=\tilde{t}}^{2 g} \lambda_{m+1, f+(t-1)(2 g)^{m}}^{n+1, k} h_{1}^{t, 0}(\pi) \\
& \quad=\mu_{2(\tilde{t}-g)-2} \pi^{-(2(\tilde{t}-g)-2)}+\cdots
\end{aligned}
$$

Then define $A_{m, f}^{n+1, k}:=A_{m, f}^{n+1, k}(x, y)-\frac{\mu}{\chi^{\tilde{t}-g-1}} x^{\tilde{t}-g-1}$.

(iv) $\tilde{t}:=\tilde{t}-1$.

(h) Repeat Step (5) (g) (i) for $\tilde{t}=g+1$.

(i) Let $m:=m-1$.

(6) For $k \in\left\{1, \ldots,(2 g)^{n+1}\right\}-\mathscr{F}_{n+1}$ do:

$$
a_{0,1}^{n+1, k}:=A_{0,1}^{n+1, k}(x, y)-A_{0,1}^{n+1, k}(x(b), y(b))
$$

(7) For $k \in\left\{1, \ldots,(2 g)^{n+1}\right\}-\mathscr{F}_{n+1}$ do:

(i) For $f \in \mathscr{F}_{n}$ define $b_{n, f}^{n+1, k}$ by

$$
b_{n, f}^{n+1, k}=a_{n, f}^{n+1, k}+s_{n, f}^{n+1, k} .
$$


(ii) For $m \in\{0, \ldots, n-1\}$ and $f \in \mathscr{F}_{m}$ define $b_{m, f}^{n+1, k}$ by

$$
b_{m, f}^{n+1, k}=a_{m, f}^{n+1, k}+s_{m, f}^{n+1, k}-\sum_{t=g+1}^{2 g} \lambda_{m+1, f+(t-1)(2 g)^{m}}^{n+1, k} h_{m+1}^{t, m} .
$$

Proof We shall now show that the lifts $S_{m}^{f}, T_{m}^{f}$ exist. Before we start, recall that in the proof of Lemma 12 we observed when taking the lifts $T_{m}^{f}, S_{m}^{f}$ that we could assume $a_{m, f}^{n+1, k}, b_{m, f}^{n+1, k}$ are both 0 if $k \in \mathscr{F}_{n+1}$. Henceforth we shall assume that $k \notin \mathscr{F}_{n+1}$. We proceed inductively with the following inductive hypothesis:

$\left(D_{m}\right)$ : For $f \in \mathscr{F}_{m}$ and $k \in\left\{1, \ldots,(2 g)^{n+1}\right\}-\mathscr{F}_{n+1}$ the following hold:

1. There exists an $A_{m, f}^{n+1, k}(x, y) \in K[x, y] /\left(y^{2}-f(x)\right)$ such that $\lambda_{m, f}^{n+1, k}:=a_{m, f}^{n+1, k}-$ $A_{m, f}^{n+1, k}(x, y)$ is constant

2. The constant $\lambda_{p, f}^{n+1, k}$ is known for $p>m+1$.

First we consider condition $\left(I_{n}\right)$. Take $f \in \mathscr{F}_{n}$ and $k \in\left\{1, \ldots,(2 g)^{n+1}\right\}-\mathscr{F}_{n+1}$. Observe that in condition $\left(I_{n}\right)$ the expression

$$
s_{n, f}^{n+1, k}:=h_{n+1}^{\psi(k, 0, n, 2 g), n} \tau(k, f, m, 2 g)-\sum_{f^{\prime} \in \mathscr{F}_{n+1}} h_{n+1}^{\psi\left(f^{\prime}, 0, n, 2 g\right), n} \tau\left(f^{\prime}, f, m, 2 g\right) \delta_{f^{\prime}, k}
$$

is known. We shall now analyse the possible orders of poles of $s_{n, f}^{n+1, k}$ at $\infty$.

Since $k \notin \mathscr{F}_{n+1}$ we conclude that $s_{m, f}^{n+1, k}=h_{n+1}^{\psi(k, 0, n, 2 g), n} \tau(k, f, m, 2 g)$. Since $f \in \mathscr{F}_{n}$ and $k \notin \mathscr{F}_{n+1}$ by Lemma 23 we conclude that $\psi(k, 0, n, 2 g) \in\{1, \ldots, g\}$ and, hence, each $h_{n+1}^{\psi(k, 0, n, 2 g), n}$ is regular at $\infty$. Therefore, each $s_{n, f}^{n+1, k}$ is regular at $\infty$.

As $b_{n, f}^{n+1, k}=a_{n, f}^{n+1, k}+s_{n, f}^{n+1, k}$ on $X \cap Y$ and both $b_{n, f}^{n+1, k}$ and $s_{n, f}^{n+1, k}$ are regular at $\infty$ we conclude that $a_{n, f}^{n+1, k}$ must be constant as all the non-constant sections in $H^{0}\left(X, \mathscr{O}_{X}\right)$ have poles of order at least 2 at $\infty$. We let $a_{n, f}^{n+1, k}=\lambda_{n, f}^{n+1, k} \in K$ be arbitrary for now.

Observe that for $f \in \mathscr{F}_{n}$ and $k \in\left\{1, \ldots,(2 g)^{n+1}\right\}-\mathscr{F}_{n+1}$ we have $A_{n, f}^{n+1, k}=0$ and $\left(D_{n}\right)$ holds trivially. Now we consider condition $\left(I_{m}\right)$. Suppose we have shown that $\left(D_{m+1}\right)$ holds. We define $s_{m, f}^{n+1, k}$ for $f \in \mathscr{F}_{m}$ and $k \notin \mathscr{F}_{n+1}$ as in Step (5)(a) of the Algorithm. Then rearranging condition $\left(I_{m}\right)$ we find that

$$
b_{m, f}^{n+1, k}=a_{m, f}^{n+1, k}+s_{m, f}^{n+1, k}-\sum_{f^{\prime} \in \mathscr{F}_{m+1}} \lambda_{m+1, f^{\prime}}^{n+1, k} h_{m+1}^{\psi\left(f^{\prime}, 0, m, 2 g\right), m} \tau\left(f^{\prime}, f, m, 2 g\right) .
$$

Observe that for $p>m+1$ the expansions in the fixed local parameter $\pi$ of $b_{p, f^{\prime}}^{n+1, k}$ appearing in $s_{m, f}^{n+1, k}$ are known. This follows from a simple induction on $p$ together with the original induction hypothesis $(D)$ upon writing $b_{p, f^{\prime}}^{n+1, k}$ as follows:

$b_{p, f^{\prime}}^{n+1, k}=A_{p, f^{\prime}}^{n+1, k}(x, y)+\lambda_{p, f^{\prime}}^{n+1, k}+s_{p, f^{\prime}}^{n+1, k}-\sum_{f^{*} \in \mathscr{F}_{m+1}} \lambda_{p+1, f^{*}}^{n+1, k} h_{m+1}^{\psi\left(f^{*}, 0, m, 2 g\right), m} \tau\left(f^{*}, f^{\prime}, m, 2 g\right)$

Similarly, since we know by our inductive hypothesis that $a_{m+1, f^{\prime}}^{n+1, k}=A_{m+1, f^{\prime}}^{n+1, k}+\lambda_{m+1, f^{\prime}}^{n+1, k}$ for some unknown constant $\lambda_{m+1, f^{\prime}}^{n+1, k}$ it follows that $b_{m+1, f^{\prime}}^{n+1, k}-\lambda_{m+1, f^{\prime}}^{n+1, k}$ is known. Therefore, we conclude that we may compute the $\pi$-expansion of $s_{m+1, f^{\prime}}^{n+1, k}$. 
From the definition of $\tau$ and Lemma 11 we may express (14) as

$$
b_{m, f}^{n+1, k}=a_{m, f}^{n+1, k}+s_{m, f}^{n+1, k}-\sum_{t=g+1}^{2 g} \lambda_{m+1, f+(t-1)(2 g)^{m}}^{n+1, k} h_{m+1}^{t, m} .
$$

Recall that $h_{m+1}^{t, m}=h_{1}^{t, 0}$ has a pole of order $2(t-g)-1$ at $\infty$. The orders of the poles at $\infty$ of the $s_{m, f}^{n+1, k}, h_{m+1}^{t, m}$ force the choices made in the above iterative process. As we vary over all $f \in \mathscr{F}_{m}$ we will compute all $\lambda_{m, f^{\prime}}^{n+1, k}$. Finally, we deduce that $a_{m, f}^{n+1, k}$ must be of the form $A_{m, f}^{n+1, k}+\lambda_{m, f}^{n+1, k}$ for some constant $\lambda_{m, f}^{n+1, k}$. So we have shown that $\left(D_{m+1}\right) \Rightarrow\left(D_{m}\right)$ and by induction $\left(D_{m}\right)$ is true for all $m$. Hence, using the above iterative process we have computed $a_{m, f}^{n+1, k}$ for all $m>0$ and $f \in \mathscr{F}_{m}$. We know for $f \in \mathscr{F}_{0}=\{1\}$ that $a_{0, f}^{n+1, k}=A_{0, f}^{n+1, k}+\lambda_{0, f}^{n+1, k}$ for some known $A_{0, f}^{n+1, k}(x, y) \in K[x, y] /\left(y^{2}-f(x)\right)$ and a constant $\lambda_{0, f}^{n+1, k} \in K$ to be determined. Since we require that $1 \in b^{*} F^{0} \overline{\mathscr{U}}_{n}$ we must have for all $k$ that $a_{0, f}^{n+1, k}(b)=0$. Hence, we require that $\lambda_{0, f}^{n+1, k}=-A_{0, f}^{n+1, k}(x(b), y(b))$.

Proof (Theorem 4) We proceed by induction. Following Example 2 we know that the statement of the theorem is true for $n=1$ so we now suppose that it is true for some $n$. That is, we have unique generators for $F^{0} \overline{\mathscr{U}}_{n}$ over $X$ and $Y$ respectively that lift generators of $F^{0} \overline{\mathscr{U}}_{n-1}$. By Lemmas 12, 13 and Algorithm 5 there exist unique generators of a bundle $F^{0} \overline{\mathscr{U}}_{n+1}$ lifting generators of $F^{0} \overline{\mathscr{U}}_{n}$ which satisfy the conditions of Lemma 10 (Hadian's lemma). For $p<0$ we now proceed to show the existence and uniqueness of $F^{p} \overline{\mathscr{U}}_{n}$ using the approach used above together with Griffith's transversality on the generators of $F^{0} \overline{\mathscr{U}}_{n}$.

\subsection{An application of Theorem 4 to elliptic curves}

In this subsection we focus on the case where $C$ is a complete elliptic curve. We apply Algorithm 5 to compute the generators of $F^{0} \overline{\mathscr{U}}_{n}$.

Remark 28 Before we proceed note that in the case that $C$ is an elliptic curve $\mathscr{F}_{n}=\left\{2^{n}\right\}$. Therefore, we can dispense with identifying a distinguished $f \in \mathscr{F}_{n}$ and, as such, we will drop the subscript $f$ throughout this section.

To describe the algorithm in question we will first give a restatement of the conditions $I_{m}$ appearing in the proof of Theorem 4 .

Lemma 14 When $C$ is an elliptic curve the conditions $I_{m}$ of Lemma 12 may be restated as follows:

$$
\begin{aligned}
& I_{n}: \text { For } k \in\left\{1, \ldots, 2^{n+1}-1\right\} \\
& \qquad \begin{aligned}
I_{m}: \text { For } k & \in\left\{1, \ldots, 2^{n+1}-1\right\} \\
b_{m}^{n+1, k}= & a_{m}^{n+1, k}+\sum_{t=1}^{2^{n+1-m}-1} h_{n+1}^{t, m} \delta_{k, t 2^{m}}+\sum_{l=m+1}^{n} a_{m}^{l, k-(\psi(k, 0, l, 2 g)-1)(2 g)^{l}} h_{n+1}^{\psi(k, 0, l, 2 g), l}+h_{n+1}^{1, n} \delta_{k, 2^{n}} \\
& -\sum_{p=m+1}^{n} b_{p}^{n+1, k} h_{p}^{2^{p-m}, m}
\end{aligned}
\end{aligned}
$$


where $m \in\{0, \ldots, n-1\}$.

Proof Recall that if $k=2^{n+1}$ then $b_{m}^{n+1, k}=a_{m}^{n+1, k}=0$ and so we consider only the cases where $k<2^{n+1}$. The remaining cases are simple to deal with by observing the following: $\tau\left(k, 2^{n}, n, 2\right)=1$ if $k=2^{n}+(\psi(k, 0, n, 2)-1) 2^{n}=\psi(k, 0, n, 2) 2^{n}$ and is 0 otherwise; since $k<2^{n+1}$ and $\psi(k, 0, n, 2) \in\{1,2\} ; \psi\left(2^{p}, 0, m, 2\right)=2^{p-m}$ by definition of $\psi$; and, finally, $\tau\left(2^{p}, 2^{m}, m, 2\right)=1$.

Using the above formulation we will now make several explicit applications of Theorem 4 to compute $F^{0} \mathscr{U}_{n}$ on an elliptic curve $C$ for several new values of $n$. The case when $n=1$ is known by Example 2. In [24, Lemma 3.2] Kim shows that $F^{0} \mathscr{U}_{2}$ is generated by $1, A_{1}, A_{1}^{2}$ and so we expect that Algorithm 5 should reproduce this as its output.

Proposition 7 The generators of $F^{0} \overline{\mathscr{U}}_{2}$ over both $X$ and $Y$ are $1, A_{1}, A_{1}^{2}$.

Proof By Proposition 4 we have that the gauge transformation $G_{2}$ is of the form

$$
G_{2}=\left(\begin{array}{cc}
I_{4 \times 4} & H_{2} \\
0_{3 \times 4} & G_{1}
\end{array}\right), \quad H_{2}=\left(\begin{array}{ccc}
0 & 0 & 0 \\
0 & 0 & 0 \\
F & 0 & 0 \\
0 & F & \frac{1}{2} F^{2}
\end{array}\right)
$$

with respect to the basis $\mathscr{B}_{2}$, where $F \in K(C)$ is such that $-\alpha_{1}+d F$ is regular at $\infty$. In Example 2 we saw that $F^{0} \overline{\mathscr{U}}_{1}$ is generated by 1 and $A_{1}$. We look for lifts of these generators over $X$ :

$$
1+\sum_{k=1}^{3} a_{0}^{2, k} w_{2}^{k}, \quad A_{1}+\sum_{k=1}^{3} a_{1}^{2, k} w_{2}^{k}, \quad A_{1}^{2}
$$

and over $Y$ :

$$
1+\sum_{k=1}^{3} b_{0}^{2, k} w_{2}^{k}, \quad A_{1}+\sum_{k=1}^{3} b_{1}^{2, k} w_{2}^{k}, \quad A_{1}^{2}
$$

where $a_{m}^{2, k} \in H^{0}\left(X, \mathscr{O}_{X}\right)$ and $b_{m}^{2, k} \in H^{0}\left(Y, \mathscr{O}_{Y}\right)$. Using Lemma 14 these sections must satisfy the following conditions on restriction to $X \cap Y$ :

$$
I_{1}: b_{1}^{2, k}=a_{1}^{2, k} \quad I_{0}: b_{0}^{2, k}=a_{0}^{2, k}-F b_{1}^{2, k}
$$

We conclude that $a_{1}^{2, k}=b_{1}^{2, k} \in K$ are both constant. Since $F$ must have a simple pole at $\infty, a_{0}^{2, k}$ cannot have a simple pole at $\infty$ and $b_{0}^{2, k}$ must be regular at $\infty$ we conclude that $a_{1}^{2, k}=b_{1}^{2, k}=0$. Therefore, $a_{0}^{2, k}=b_{0}^{2, k} \in K$ are constant and following Step (6) of Algorithm 5 we conclude these sections must also be 0 . Therefore, $a_{m}^{2, k}=b_{m}^{2, k}=0$ for all $k$ and we are done.

Proposition 8 The generators of $F^{0 \overline{\mathscr{U}}_{3}}$ over both $X$ and $Y$ are

$$
1, A_{1}+\lambda\left[\left[A_{0}, A_{1}\right], A_{1}\right], A_{1}^{2}, A_{1}^{3}
$$

where $\left[A_{i}, A_{j}\right]=A_{i} A_{j}-A_{j} A_{i}$ is the commutator bracket and $\lambda$ is as in Proposition 5.

Proof This is a straightforward application using Proposition 5, Algorithm 5, Lemma 14 and the filtration computed in Proposition 7. 
Remark 29 It is at this stage that we see some dependency in the Hodge filtration on the choice of basis of $H_{d r}^{1}(X)$. If we let $\alpha_{0}=\frac{d x}{y}$ and $\alpha_{1}=\frac{x d x}{y}$ then we may take $F=\frac{y}{x}$. In this case $\lambda=-2$.

Proposition 9 The generators of $F^{0} \mathscr{U}_{4}$ as an $\mathscr{O}_{X}$-module are

$$
\begin{aligned}
& 1+\frac{1}{3} v(x-x(b))\left[A_{1},\left[A_{1},\left[A_{1}, A_{0}\right]\right]\right] \\
& A_{1}+\lambda\left[\left[A_{0}, A_{1}\right], A_{1}\right]-\left(\mu+\frac{1}{3} \kappa\right)\left[A_{1},\left[A_{1},\left[A_{1}, A_{0}\right]\right]\right] \\
& A_{1}^{2}+\lambda\left[\left[A_{0}, A_{1}\right], A_{1}^{2}\right], \quad A_{1}^{3}, \quad A_{1}^{4}
\end{aligned}
$$

where $\lambda, \mu$ are as in Proposition 6 and $\kappa, v \in K$ are constants such that $v x+\lambda F^{2}$ and $v x+\kappa F+\lambda F^{2}$ are regular at $\infty$.

Proof We again proceed by application of Proposition 6 to compute $G_{4}$, Algorithm 5 and Lemma 14. We determine sections $a_{m}^{4, k} \in H^{0}\left(X, \mathscr{O}_{X}\right)$ defining the generators as in the algorithm. These are displayed in the following table:

\begin{tabular}{lll}
\hline$m=$ & $k$ & $a_{m}^{4, k}=$ \\
\hline 3 & All $k$ & 0 \\
2 & $\neq 8,12,14,15$ & 0 \\
& $=8$ & $\lambda$ \\
& $=12$ & $-\lambda$ \\
& $=14$ & $-\lambda$ \\
& $=15$ & $\lambda$ \\
& $\neq 8,12,14,15$ & 0 \\
& $=8$ & $\mu+\frac{1}{3} \kappa$ \\
& $=12$ & $-3 \mu-\kappa$ \\
& $=14$ & $3 \mu+\kappa$ \\
& $=15$ & $-\mu-\frac{1}{3} \kappa$ \\
0 & $\neq 8,12,14,15$ & 0 \\
& $=8$ & $-\frac{1}{3} v(x-x(b))$ \\
& $=12$ & $\nu(x-x(b))$ \\
& $=14$ & $-v(x-x(b))$ \\
& $=15$ & $\frac{1}{3} v(x-x(b))$ \\
\hline
\end{tabular}

It is then a simple case of expressing them in terms of the commutator brackets.

Remark 30 If we again take $\alpha_{0}=\frac{d x}{y}, \alpha_{1}=\frac{x d x}{y}$ and $F=\frac{y}{x}$ then we will find that $\mu=\kappa=0$, $\lambda=2$ and $v=8$.

\subsection{An application of Theorem 4 to odd hyperelliptic curves}

In this section we focus on the case where $C$ is a complete odd hyperelliptic curve. We focus on providing a complete description of the generators of $F^{0} \mathscr{U}_{2}$. First we provide a more suitable restatement of the conditions $I_{m}$ appearing at level 2 .

Lemma 15 The conditions $I_{m}$ of Lemma 12 when $n=1$ may be restated as follows: 
$I_{1}:$ For $k \in\left\{1, \ldots,(2 g)^{n+1}\right\}-\mathscr{F}_{2}$ and $f \in \mathscr{F}_{1}$

$$
b_{1, f}^{2, k}=a_{1, f}^{2, k}+h_{2}^{k_{1}+1,1} \delta_{1+k_{0}, f}
$$

$I_{0}:$ For $k \in\left\{1, \ldots,(2 g)^{n+1}\right\}-\mathscr{F}_{2}$

$$
b_{0,1}^{2, k}=a_{0,1}^{2, k}+h_{2}^{k, 0}-\sum_{f=g+1}^{2 g} b_{1, f}^{2, k} h_{1}^{f, 0}
$$

where $k=1+k_{0}+k_{1}(2 g)$ for some $k_{0}, k_{1} \in\{0, \ldots, 2 g-1\}$.

Proof The proof of this lemma rests on a few simple computations. First we have $\psi(k, 0,0,2 g)=k$ by definition of $\psi$. Therefore, by definition of $\tau$ we find that $\tau(k, 1,0,2 g)=1$ since $k=1+(k-1)(2 g)^{0}$. Second, observe that $k=1+k_{0}+k_{1}(2 g)$ for some unique $k_{0}, k_{1} \in\{0, \ldots, 2 g-1\}$. It is easy then to check that $\psi(k, 0,1,2 g)=k_{1}+1$. Therefore, we find that $\tau(k, f, 1,2 g)=1$ if and only if $k=f+k_{1}(2 g)$ and is 0 otherwise. Putting all of these calculations together we deduce the formulation of the conditions $I_{0}$ and $I_{1}$ as in the statement of the lemma.

Using this formulation of the conditions $I_{1}$ and $I_{0}$ we can prove the following proposition.

Proposition 10 The generators of $F^{0} \mathscr{U}_{2}$ as an $\mathscr{O}_{X}$-module are

$$
\begin{gathered}
1+\sum_{\substack{0 \leq i<g \\
g \leq j<2 g}} a_{i j}\left[A_{i}, A_{j}\right] \\
A_{k}+\sum_{\substack{0 \leq i<g \\
g \leq j<2 g}} c_{i j k}\left[A_{i}, A_{j}\right]
\end{gathered}
$$

$A_{r} A_{s}$

where $k, r, s \in\{g, \ldots, 2 g-1\}$ and $a_{i j}, c_{i j k} \in H^{0}\left(X, \mathscr{O}_{X}\right)$ are sections such that

(1) $c_{i j k}$ are constant for all $i, j, k$

(2) $a_{i j}$ are such that

$$
a_{i j}+h_{2}^{1+j+i(2 g), 0}-\sum_{f=g+1}^{2 g} c_{i j k} h_{1}^{f, 0}
$$

are regular at $\infty$ and evaluate to 0 at $b$.

Proof We proceed by applying Algorithm 5 with the reformulation of the conditions as in Lemma 15. First we consider condition $I_{1}$. We need to examine the term $h_{2}^{k_{1}+1,1} \delta_{1+k_{0}, f}=$ $h_{1}^{k_{1}+1,0} \delta_{1+k_{0}, f}$. Since $k \notin \mathscr{F}_{2}$ and $f \in \mathscr{F}_{1}$ by Lemma $111+k_{0}=f$ can only occur when $k_{1}<g$. Hence $k_{1}+1 \in\{1, \ldots, g\}$. Therefore, when they appear, each $h_{2}^{k_{1}+1,1}$ must be regular at $\infty$ and hence each $a_{1, f}^{2, k}$ is regular at $\infty$ and hence constant on $C$.

We now examine condition $I_{0}$. Observe that $c_{i j k}=a_{1, k+1}^{2,1+j+i(2 g)}$ is the coefficient of $A_{i} A_{j}$ in the lift of the generator $A_{k}$ of $F^{0} \mathscr{U}_{1}$ and that $a_{i j}=a_{0,1}^{2,1+j+i(2 g)}$ is the coefficient of $A_{i} A_{j}$ in the lift of 1 . To complete the proof of the proposition we, therefore, need to show: 


$$
\begin{gathered}
c_{i j k}=-c_{j i k} \text { for all } k, i, j \text { and } c_{i j k}=0 \text { if } i, j<g \\
a_{i j}=-a_{j i} \text { for all } i, j \text { and } a_{i j}=0 \text { if } i, j<g
\end{gathered}
$$

Condition $I_{0}$ for $k=1+j+i(2 g), 1+i+j(2 g)$ are

$$
\begin{aligned}
& b_{0,1}^{2,1+j+i(2 g)}=a_{i j}+h_{2}^{1+j+i(2 g), 0}-\sum_{f=g+1}^{2 g} b_{1, f}^{2,1+j+i(2 g)} h_{1}^{f, 0} \\
& b_{0,1}^{2,1+i+j(2 g)}=a_{j i}+h_{2}^{1+i+j(2 g), 0}-\sum_{f=g+1}^{2 g} b_{1, f}^{2,1+i+j(2 g)} h_{1}^{f, 0} .
\end{aligned}
$$

By Algorithm 3 we know that $h_{2}^{1+j+i(2 g), 0}$ and $h_{2}^{1+i+j(2 g), 0}$ are such that

$$
\begin{aligned}
& d h_{2}^{2 g i+j+1,0}-\alpha_{i} h_{1}^{j+1,0}-c_{1}^{j+1,0} h_{1}^{i+1,0} \\
& d h_{2}^{2 g j+i+1,0}-\alpha_{j} h_{1}^{i+1,0}-c_{1}^{i+1,0} h_{1}^{j+1,0}
\end{aligned}
$$

have at worst logarithmic poles at $\infty$. Here $c_{1}^{j+1,0}=d h_{1}^{j+1,0}-\alpha_{j}$.

Now if $i, j<g$ then $\alpha_{i}, \alpha_{j} \in \Omega_{C / K}^{1}$ are regular on $C$. Therefore, $h_{1}^{i+1,0}, h_{1}^{j+1,0}$ must be regular at $\infty$ and hence $h_{2}^{1+j+i(2 g), 0}$ and $h_{2}^{1+i+j(2 g), 0}$ must also be regular at $\infty$. Applying Algorithm 5 we conclude that when $i, j<g$ then $c_{i j k}=0$ for all $k$ and that $a_{i j}$ are constant. Since they must evaluate to 0 at $b$ we conclude that they too are all 0 .

Suppose now that $i<g$ and $j \geq g$. Adding conditions in (17) and (18) together we obtain $d\left(h_{2}^{2 g i+j+1,0}+h_{2}^{2 g j+i+1,0}-h_{1}^{i+1,0} h_{1}^{j+1,0}\right)$. Hence $h_{2}^{2 g i+j+1,0}+h_{2}^{2 g j+i+1,0}-h_{1}^{i+1,0} h_{1}^{j+1,0}$ must be regular at $\infty$. If we now add (15) and (16) together and use condition $I_{1}$ we obtain

$$
\begin{aligned}
b_{0,1}^{2,1+j+i(2 g)}+b_{0,1}^{2,1+i+j(2 g)}= & a_{0,1}^{2,1+j+i(2 g)}+a_{0,1}^{2,1+i+j(2 g)} \\
& -\sum_{f=g+1}^{2 g}\left(a_{1, f}^{2,1+j+i(2 g)}+a_{1, f}^{2,1+i+j(2 g)}\right) h_{1}^{f, 0} \\
& +h_{2}^{1+j+i(2 g), 0}+h_{2}^{1+i+j(2 g), 0}-h_{1}^{i+1,0} h_{1}^{j+1,0} .
\end{aligned}
$$

As before, we conclude that we must have $c_{i j k}+c_{j i k}=0$ and $a_{i j}+a_{j i}$ constant for all $i<g, j \geq g$. Since $a_{i j}+a_{j i}$ must evaluate to 0 at $b$ we conclude that this too is exactly 0 . Thus, we have shown that the lifts satisfy the conditions claimed in the statement of the proposition.

We conclude this section with an explicit example of the above lemma for genus 2 odd hyperelliptic curves.

Example 3 Let $C$ be a hyperelliptic curve of genus 2 over $K$ with odd affine model $y^{2}=f(x)$ for some $f(x) \in K[x]$ where $\operatorname{deg} f=5$. Let us take $\alpha_{i}:=\frac{x^{i} d x}{y}$, the standard $K$-basis for $H_{d r}^{1}(X / K)$. Let $F:=\frac{y}{x^{2}} \in K(C)$ and note that it has a logarithmic pole at $\infty$. Applying Algorithms 3 and 5 we conclude that $F^{0} \mathscr{U}_{2}$ has the following generators as an $\mathscr{O}_{X}$-module lifting the generators of $F^{0} \mathscr{U}_{1}$ :

$$
1-\frac{2}{3}(x-x(b))\left[A_{1}, A_{3}\right], A_{2}, A_{3}, A_{2}^{2}, A_{2} A_{3}, A_{3} A_{2}, A_{3}^{2}
$$




\section{Computing the de Rham period maps}

This section is concerned with explicit computation of the $p$-adic de Rham period maps introduced by Kim in [23]. As outlined in loc.cit. the coordinates of these maps can be described in terms of explicit $p$-adic iterated Coleman integrals. We will make use of the algorithms and results from Sects. 3 and 4 to compute coordinates of some of these maps at new levels as well as providing a conjectural closed form for the map on elliptic curves. First, we recall how the period map is defined.

\subsection{Defining the de Rham period map $j_{n}^{d r}$}

Throughout this section we shall assume the following: $K$ is a number field, $v$ is a nonArchimedean valuation on $K$ and $K_{v}$ is the completion of $K$ with respect to $v$. Let $C$ be an elliptic or odd hyperelliptic curve and define $X:=C-\{\infty\}$. We let $X_{v}:=X \otimes K_{v}$ denote the basechange of $X$. Take a rational basepoint $b \in X(K)$ and suppose that $x \in X_{v}\left(K_{v}\right)$. We wish to find explicit descriptions for the maps

$$
j_{n}^{d r}: X_{v}\left(K_{v}\right) \rightarrow F^{0} U_{n}^{d r} \backslash U_{n}^{d r} .
$$

Recall from Sect. 2 that in order to compute $j_{n}^{d r}(x)$ we need to identify the following trivialisations over $K_{v}$ : a Frobenius trivialisation $p_{n}^{c r}(x) \in P_{n}^{d r}(x)$; a Hodge trivialisation $p_{n}^{H}(x) \in F^{0} P_{n}^{d r}(x)$; and a trivialisation $u_{n}(x) \in U_{n}^{d r}$ such that $p_{n}^{c r}(x)=p_{n}^{H}(x) u_{n}(x)$. We know that

$$
p_{n}^{c r}(x)=1+\sum_{|w| \leq n} \int_{b}^{x} \alpha_{w} w
$$

where the sum is taken over all words of length at most $n$. We have shown in Sect. 4 that $\mathscr{U}_{n}$ possesses a Hodge filtration $F^{\bullet} \mathscr{U}_{n}$. This induces a filtration on the dual bundles $\mathscr{U}_{n}^{\vee}$ : define $F^{i}\left(\mathscr{U}_{n}^{\vee}\right):=\left(F^{1-i} \mathscr{U}_{n}\right)^{\perp}=\left\{l \in \mathscr{U}_{n}^{\vee} \mid F^{1-i}\left(\mathscr{U}_{n}\right) \subseteq \operatorname{ker} l\right\}$. Recall that $F^{-1+i} \mathscr{P}_{n}^{d r}$ is the defining ideal for $F^{i} P_{n}^{d r}$. Therefore, $F^{i} P_{n}^{d r}$ has defining ideal $\left(F^{i} \mathscr{U}_{n}\right)^{\perp}$, or $F^{i} P_{n}^{d r}=$ $\operatorname{Spec}\left(\mathscr{U}_{n}^{\vee} /\left(F^{i} \mathscr{U}_{n}\right)^{\perp}\right)$. Therefore, arguing as in Proposition 1 the group-like elements of $x^{*} F^{i} \mathscr{U}_{n}$ correspond to elements of $F^{i} P_{n}^{d r}(x)$. We utilise this property in what follows.

The first explicit description of the de Rham period maps beyond level 1 was given in [10] for $n=2$ when $C$ is an elliptic curve.

Proposition 11 ([10], Proof of Corollary 0.2') The level 2 unipotent Albanese map $j_{2}^{d r}$ : $X_{v}\left(K_{v}\right) \rightarrow F^{0} U_{2}^{d r} \backslash U_{2}^{d r}$ is defined by

$$
x \mapsto \int_{b}^{x} \alpha_{0} A_{0}+\int_{b}^{x} \alpha_{0} \alpha_{1}\left[A_{0}, A_{1}\right] .
$$

Proof The point is that we know $1, A_{1}, A_{1}^{2}$ generate $F^{0} \mathscr{U}_{2}$ using Proposition 7. It is a simple computation to check that

$$
p_{2}^{c r}(x)=\exp \left(\int_{b}^{x} \alpha_{1} A_{1}\right) \exp \left(\int_{b}^{x} \alpha_{0} A_{0}+\int_{b}^{x} \alpha_{0} \alpha_{1}\left[A_{0}, A_{1}\right]\right) .
$$

Note that $\int_{b}^{x} \alpha_{0} A_{0}+\int_{b}^{x} \alpha_{0} \alpha_{1}\left[A_{0}, A_{1}\right]$ is primitive since linear combinations and commutators of primitive elements are primitive. We conclude that we have found the decomposition $p_{2}^{c r}(x)=p_{2}^{H}(x) u_{2}(x)$ as required. 
Remark 31 Observe that the map of the above proposition is actually the logarithm of $j_{2}^{d r}$. As per Remark 9, however, there is a bijection between $\operatorname{Lie} U_{2}^{d r}$ and $U_{2}^{d r}$ coming from the exp and log maps. For the sake of brevity it will be more convenient here and in what follows to present the image of the map in $\operatorname{Lie} U_{n}^{d r}$. However, we will suppress this in the notation, making implicit use of the previously stated bijective correspondence.

Remark 32 Note that in order to compute the image of the map $j_{2}^{d r}$ for specific values of $x \in X_{v}\left(K_{v}\right)$ we need to compute the iterated integrals $\int_{b}^{x} \alpha_{0}$ and $\int_{b}^{x} \alpha_{0} \alpha_{1}$. These can be computed using the algorithms of Balakrishnan in [3]; this involves computation of the matrix of action of Frobenius on $H_{d r}^{1}(X / K)$ by making use of Kedlaya's algorithm and solving a system of linear equations to compute the integrals between Teichmüller points.

In what follows we will make use of two properties of iterated integrals ([2, Proposition 5.2.1]):

$$
\begin{aligned}
\int \omega_{1} \ldots \omega_{r} \int \omega_{r+1} \ldots \omega_{s} & =\sum_{\sigma \in S(r, s)} \int \omega_{\sigma(1)} \ldots \omega_{\sigma(s)} \\
\int \underbrace{\omega \omega \ldots \omega}_{n \text { times }} & =\frac{1}{n !}\left(\int \alpha\right)^{n}
\end{aligned}
$$

where in (21) we sum over all permutations of shuffle type $(r, s)$. Note that (22) is a simple consequence of (21).

\subsection{The levels 3 and 4 period maps on affine elliptic curves}

In this section we will compute the levels 3 and 4 maps on affine elliptic curves using the results of the previous two sections. Recall from Proposition 8 the Hodge filtration of $F^{0} \mathscr{U}_{3}$ is generated by

$$
1, A_{1}+\lambda\left[\left[A_{0}, A_{1}\right], A_{1}\right], A_{1}^{2}, A_{1}^{3} \text {. }
$$

We want to find a primitive $p_{3}^{H}(x)$ in the $K_{v}$-algebra generated by these, and a primitive $u_{3}(x) \in x^{*} \mathscr{U}_{3}$ such that $p_{3}^{c r}(x)=\exp \left(p_{3}^{H}(x)\right) \exp \left(u_{3}(x)\right)$. We may then define $j_{3}^{d r}(x)=$ $u_{3}(x)$.

Proposition 12 The level 3 unipotent Albanese map $j_{3}^{d r}: X_{v}\left(K_{v}\right) \rightarrow F^{0} U_{3}^{d r} \backslash U_{3}^{d r}$ is defined by

$$
\begin{aligned}
x \mapsto u_{3}(x):= & \int_{b}^{x} \alpha_{0} A_{0}+\int_{b}^{x} \alpha_{0} \alpha_{1}\left[A_{0}, A_{1}\right]+\frac{1}{2} \int_{b}^{x} \alpha_{0} \alpha_{1} \alpha_{0}\left[A_{0},\left[A_{1}, A_{0}\right]\right] \\
& +\int_{b}^{x}\left(\alpha_{0} \alpha_{1} \alpha_{1}-\lambda \alpha_{1}\right)\left[\left[A_{0}, A_{1}\right], A_{1}\right]
\end{aligned}
$$

Proof Using properties (21),(22) of iterated integrals and by comparing $p_{3}^{c r}(x)$ to

$$
\exp \left(\int_{b}^{x}\left(\alpha_{1} A_{1}+\lambda\left[\left[A_{0}, A_{1}\right], A_{1}\right]\right)\right) \exp \left(\int_{b}^{x} \alpha_{0} A_{0}+\int_{b}^{x} \alpha_{0} \alpha_{1}\left[A_{0}, A_{1}\right]\right)
$$


we can rewrite $p_{3}^{c r}(x)$ as follows

$$
\begin{aligned}
p_{3}^{c r}(x)= & +\int_{b}^{x} \alpha_{0} A_{0}+\int_{b}^{x} \alpha_{1} A_{1}+\frac{1}{2}\left(\int_{b}^{x} \alpha_{0}\right)^{2} A_{0}^{2}+\int_{b}^{x} \alpha_{0} \alpha_{1} A_{0} A_{1} \\
& +\left(\int_{b}^{x} \alpha_{1} \int_{b}^{x} \alpha_{0}-\int_{b}^{x} \alpha_{0} \alpha_{1}\right) A_{1} A_{0}+\left(\int_{b}^{x} \alpha_{1} \int_{b}^{x} \alpha_{0} \alpha_{1}-2 \lambda \int_{b}^{x} \alpha_{1}\right) A_{1} A_{0} A_{1} \\
& +\left(\frac{1}{2}\left(\int_{b}^{x} \alpha_{0}\right)^{2} \int_{b}^{x} \alpha_{1}-\frac{1}{2} \int_{b}^{x} \alpha_{0} \int_{b}^{x} \alpha_{0} \alpha_{1}\right) A_{1} A_{0}^{2} \\
& +\left(\frac{1}{2}\left(\int_{b}^{x} \alpha_{1}\right)^{2} \int_{b}^{x} \alpha_{0}-\int_{b}^{x} \alpha_{1} \int_{b}^{x} \alpha_{0} \alpha_{1}+\lambda \int_{b}^{x} \alpha_{1}\right) A_{1}^{2} A_{0}+\frac{1}{3 !}\left(\int_{b}^{x} \alpha_{1}\right)^{3} A_{1}^{3} \\
& +\frac{1}{2} \int_{b}^{x} \alpha_{0} \alpha_{1} \alpha_{0}\left[A_{0},\left[A_{1}, A_{0}\right]\right]+\left(\int_{b}^{x} \alpha_{0} \alpha_{1} \alpha_{1}-\lambda \int_{b}^{x} \alpha_{1}\right)\left[\left[A_{0}, A_{1}\right], A_{1}\right] \\
& =\exp \left(\int_{b}^{x} \alpha_{1}\left(A_{1}+\lambda\left[\left[A_{0}, A_{1}\right], A_{1}\right]\right)\right) \exp \left(u_{3}(x)\right)
\end{aligned}
$$

where $u_{3}(x)$ is as in the statement of the proposition. Since $A_{0}, A_{1}$ are primitive and commutators of primitives are primitive we are done.

To compute the level 4 map we try to mimic the approach taken at level 4 by comparing a modification of the decomposition at level 3 to the Frobenius invariant element at level 4.

Proposition 13 The level 4 unipotent Albanese map $j_{4}^{d r}: X_{v}\left(K_{v}\right) \rightarrow F^{0} U_{4}^{d r} \backslash U_{4}^{d r}$ is defined by

$$
\begin{aligned}
x \mapsto u_{4}(x):= & \int_{b}^{x} \alpha_{0} A_{0}+\int_{b}^{x} \alpha_{0} \alpha_{1}\left(\left[A_{0}, A_{1}\right]+\frac{\lambda}{2}\left[\left[A_{0},\left[A_{0}, A_{1}\right]\right], A_{1}\right]\right) \\
& +\frac{1}{2} \int_{b}^{x} \alpha_{0} \alpha_{1} \alpha_{0}\left[A_{0},\left[A_{1}, A_{0}\right]\right] \\
& +\int_{b}^{x}\left(\alpha_{0} \alpha_{1} \alpha_{1}-\lambda \alpha_{1}\right)\left[\left[A_{0}, A_{1}\right], A_{1}\right] \\
& +\frac{1}{6} \int_{b}^{x} \alpha_{0} \alpha_{0} \alpha_{1} \alpha_{0}\left[\left[A_{0},\left[A_{0}, A_{1}\right]\right], A_{0}\right] \\
& +\frac{1}{6} \int_{b}^{x} \alpha_{0} \alpha_{1} \alpha_{0} \alpha_{0}\left[\left[\left[A_{0}, A_{1}\right], A_{0}\right], A_{0}\right] \\
& +\frac{1}{2} \int_{b}^{x}\left(\alpha_{0} \alpha_{1} \alpha_{0} \alpha_{1}-\lambda \alpha_{1} \alpha_{0}\right)\left[\left[A_{0},\left[A_{1}, A_{0}\right]\right], A_{1}\right] \\
& +\int_{b}^{x}\left(\alpha_{0} \alpha_{1} \alpha_{1} \alpha_{1}-\lambda \alpha_{1} \alpha_{1}-\left(\mu+\frac{\kappa}{3}\right) \alpha_{1}\right)\left[\left[\left[A_{0}, A_{1}\right], A_{1}\right], A_{1}\right] \\
& +\frac{1}{2} \int_{b}^{x} \alpha_{0} \alpha_{1} \alpha_{0} \alpha_{1}\left[\left[\left[A_{0}, A_{1}\right], A_{1}\right], A_{0}\right]
\end{aligned}
$$

where $\lambda, \mu, \kappa$ are as in Proposition 9.

Proof We proceed by a comparison of $p_{4}^{c r}(x)$ with

$$
\exp \left(\int_{b}^{x} \alpha_{1}\left(A_{1}+\lambda\left[\left[A_{0}, A_{1}\right], A_{1}\right]-\left(\mu+\frac{1}{3} \kappa\right)\left[A_{1},\left[A_{1},\left[A_{1}, A_{0}\right]\right]\right]\right)\right) \exp \left(u_{3}(x)\right) .
$$


A computation similar to that in the proof of Proposition 12 shows that

$p_{4}^{c r}(x)=\exp \left(\int_{b}^{x} \alpha_{1}\left(A_{1}+\lambda\left[\left[A_{0}, A_{1}\right], A_{1}\right]-\left(\mu+\frac{\kappa}{3}\right)\left[A_{1},\left[A_{1},\left[A_{1}, A_{0}\right]\right]\right]\right)\right) \exp \left(u_{4}(x)\right)$

where $u_{4}(x)$ is as in the statement of the proposition. Therefore since the coefficient of $\int_{b}^{x} \alpha_{1}$ is primitive and a generator of $F^{0} \mathscr{U}_{4}$ by Proposition 9 we are done.

\subsection{A useful lemma to compute period maps}

The examples of the previous subsection suggest the following useful lemma to compute the maps $j_{n+1}^{d r}$. Of course, this will rely on us having already computed $F^{0} \mathscr{U}_{n}$ using Algorithm 5 , as well as the co-ordinates of the map $j_{n-1}^{d r}$. We suppose we have already computed some decomposition $p_{n-1}^{c r}(x)=p_{n-1}^{H}(x) u_{n-1}(x)$. Provided a certain lifting condition is satisfied, we can describe the decomposition at level $n$ exactly.

Lemma 16 Suppose that $B_{n-1}$ is primitive in $F^{0} \mathscr{U}_{n-1}$ and that $p_{n-1}^{c r}(x)=\exp \left(\int_{b}^{x} \alpha B_{n-1}(x)\right)$ $\exp \left(j_{n-1}^{d r}(x)\right)$ for some $\alpha$ a linear combination of $\alpha_{w}$. Then suppose that the lift $B_{n}$ of $B_{n-1}$ to $F^{0} \mathscr{U}_{n}$ is also primitive. Then $j_{n}^{d r}$ has coordinates

$$
j_{n}^{d r}(x):=j_{n-1}^{d r}(x)+p_{n}^{c r}(x)-\exp \left(\int_{b}^{x} \alpha B_{n}(x)\right) \exp \left(j_{n-1}^{d r}(x)\right) .
$$

Proof Suppose that we have a decomposition

$$
p_{n-1}^{c r}(x)=\exp \left(\int_{b}^{x} \alpha B_{n-1}(x)\right) \exp \left(j_{n-1}^{d r}(x)\right) .
$$

As we did in the proofs of Propositions 12 and 13 consider the difference

$$
\begin{aligned}
& p_{n}^{c r}(x)-\exp \left(\int_{b}^{x} \alpha B_{n}(x)\right) \exp \left(j_{n-1}^{d r}(x)\right) \\
& \quad=p_{n}^{c r}(x)-\exp \left(\int_{b}^{x} \alpha\left(B_{n}(x)-B_{n-1}(x)\right)\right) \exp \left(\int_{b}^{x} \alpha B_{n-1}(x)\right) \exp \left(j_{n-1}^{d r}(x)\right) .
\end{aligned}
$$

Since we have the previously stated decomposition (23) at level $n-1$ then

$$
\exp \left(\int_{b}^{x} \alpha B_{n-1}(x)\right) \exp \left(j_{n-1}^{d r}(x)\right)=p_{n-1}^{c r}(x)+\text { words of degree } n .
$$

Therefore (24) contains only words of length $n$. Therefore

$$
\exp \left(p_{n}^{c r}(x)-\exp \left(\int_{b}^{x} \alpha B_{n}(x)\right) \exp \left(j_{n-1}^{d r}(x)\right)\right)
$$

commutes with $\exp \left(\int_{b}^{x} \alpha B_{n}(x)\right) \exp \left(j_{n-1}^{d r}(x)\right)$ and we find that

$$
\begin{aligned}
& \exp \left(\int_{b}^{x} \alpha B_{n}(x)\right) \exp \left(j_{n-1}^{d r}(x)+p_{n}^{c r}(x)-\exp \left(\int_{b}^{x} \alpha B_{n}(x)\right) \exp \left(j_{n-1}^{d r}(x)\right)\right) \\
& =\exp \left(\int_{b}^{x} \alpha B_{n}(x)\right) \exp \left(j_{n-1}^{d r}(x)\right) \exp \left(p_{n}^{c r}(x)-\exp \left(\int_{b}^{x} \alpha B_{n}(x)\right) \exp \left(j_{n-1}^{d r}(x)\right)\right)
\end{aligned}
$$




$$
\begin{aligned}
= & \left(p_{n}^{c r}(x)+\exp \left(\int_{b}^{x} \alpha B_{n}(x)\right) \exp \left(j_{n-1}^{d r}(x)\right)-p_{n}^{c r}(x)\right) \\
& \times \exp \left(p_{n}^{c r}(x)-\exp \left(\int_{b}^{x} \alpha B_{n}(x)\right) \exp \left(j_{n-1}^{d r}(x)\right)\right) \\
= & p_{n}^{c r}(x)+\exp \left(\int_{b}^{x} \alpha B_{n}(x)\right) \exp \left(j_{n-1}^{d r}(x)\right)-p_{n}^{c r}(x)+p_{n}^{c r}(x) \\
& -\exp \left(\int_{b}^{x} \alpha B_{n}(x)\right) \exp \left(j_{n-1}^{d r}(x)\right) \\
= & p_{n}^{c r}(x)
\end{aligned}
$$

This gives us a decomposition of the form

$p_{n}^{c r}(x)=\exp \left(\int_{b}^{x} \alpha B_{n}(x)\right) \exp \left(j_{n-1}^{d r}(x)+p_{n}^{c r}(x)-\exp \left(\int_{b}^{x} \alpha B_{n}(x)\right) \exp \left(j_{n-1}^{d r}(x)\right)\right)$

Since $B_{n}$ is primitive then $\exp \left(\int_{b}^{x} \alpha B_{n}(x)\right)$ is group-like and we conclude that we can define $j_{n}^{d r}$ as in the statement of the theorem.

\subsection{The level 2 period map on affine hyperelliptic curves}

We can use the computation of the Hodge filtration on $\mathscr{U}_{2}$ for a generic genus $g$ odd hyperelliptic curve $C$ that we determined in Proposition 10 to determine the level 2 de Rham period map on $X:=C-\{\infty\}$. Fix a basepoint $b \in X(K)$. As above, we will find that

$$
p_{2}^{c r}(x)=1+\sum_{k=0}^{2 g-1} \int_{b}^{x} \alpha_{k} A_{k}+\sum_{k, l=0}^{2 g-1} \int_{b}^{x} \alpha_{k} \alpha_{l} A_{k} A_{l}
$$

Proposition 14 The map $j_{2}^{d r}: X_{v}\left(K_{v}\right) \rightarrow F^{0} U_{2}^{d r} \backslash U_{2}^{d r}$ is defined by

$$
\begin{aligned}
x \mapsto u_{2}(x):= & \sum_{k=0}^{g-1} \int_{b}^{x} \alpha_{k} A_{k}+\frac{1}{2} \sum_{k, l=0}^{g-1} \int_{b}^{x} \alpha_{k} \alpha_{l}\left[A_{k}, A_{l}\right]+\sum_{k=0}^{g-1} \sum_{l=g}^{2 g-1} \int_{b}^{x} \alpha_{k} \alpha_{l}\left[A_{k}, A_{l}\right] \\
& +\sum_{k=g}^{2 g-1} \sum_{\substack{0 \leq i<g \\
g \leq j<2 g}} c_{i j k} \int_{b}^{x} \alpha_{k}\left[A_{j}, A_{i}\right]
\end{aligned}
$$

Proof A careful computation shows that

$$
\begin{aligned}
p_{2}^{c r}(x)=\exp & \left(\sum_{k=g}^{2 g-1} \int_{b}^{x} \alpha_{k}\left(A_{k}+\sum_{\substack{0 \leq i<g \\
g \leq j<2 g}} a_{1, k+1}^{2,1+j+i(2 g)}\left[A_{i}, A_{j}\right]\right)\right. \\
& \left.+\frac{1}{2} \sum_{l, k=g}^{2 g-1} \int_{b}^{x} \alpha_{k} \alpha_{l}\left[A_{k}, A_{l}\right]\right) \\
& \times \exp \left(u_{2}(x)\right)
\end{aligned}
$$

where $u_{2}(x)$ is as in the statement of the proposition and we are done. 


\section{De Rham period maps with tangential basepoints}

\subsection{Computing iterated integrals with tangential basepoints}

Throughout Sects. 2 to 5 we have assumed that the basepoint $b$ is a $K$-rational point on $X$. It may be the case that we cannot readily determine such a rational basepoint. Indeed part of the value of studying the unipotent Albanese map is that they should give us the means to compute the rational or $S$-integral points on a curve (for some finite set of primes $S$ ). Therefore, we need to find a way to circumvent the obstacle of finding a rational basepoint and this is afforded to us by the theory of tangential basepoints or basepoints at infinity. These basepoints correspond to tangent vectors at the points of $D$ and philosophically speaking they should give us access to a wider range of canonical maps. To replace the rational basepoint $b$ in the maps $j_{n}^{d r}$ with a tangential basepoint will require a method of "analytic continuation" to the tangential basepoint. We briefly outline the method by which we can compute this, following the description given by Besser and Furusho in [12].

We assume once more that $C$ is a smooth projective curve over $K$ a number field punctured at a single point $P$ defined over $K$ and let $X:=C-\{P\}$. Let $v$ be a non-Archimedean valuation on $K$ and take $K_{v}$ the completion of $K$ at $v$. Let $\mathscr{U}$ be the universal unipotent connection on $X_{v}$, and $\overline{\mathscr{U}}$ its logarithmic extension to $C_{v}$. Let $t$ be a local parameter at $P$ inducing a parameter $\bar{t}$ on $T_{P} C$, which is a normal vector at $P$ taking the value 1 at the tangent vector $b=\frac{d}{d t}$. Let $T_{P}^{0} C:=T_{P} C-\{0\}$. Then we can associate to each logarithmic connection $\overline{\mathscr{V}}$ on $C$ with logarithmic poles at $P$ a connection on $T_{P}^{0} C$ :

Definition 25 Define $\operatorname{Res}_{P} \overline{\mathscr{V}}:=\left(P^{*} \overline{\mathscr{V}} \otimes \mathscr{O}_{T_{P}^{0} C}, d+\operatorname{Res}_{P} \Omega d \log \bar{t}\right)$ where $\Omega=\left(\omega_{i j}\right)$ is the connection matrix of $\overline{\mathscr{V}}$ near $P$ and $\operatorname{Res}_{P} \Omega=\left(\operatorname{res}_{P} \omega_{i j}\right)$ is the residue matrix of $\Omega$ at $P$.

In $\left[18, \S 15\right.$, Théorie algébrique] Deligne shows that the definition of $\operatorname{Res}_{P}$ does not depend on the choice of local parameter $t$ at $P$. The map $\operatorname{Res}_{P}$ gives us a functor $\operatorname{Res}_{P}: \operatorname{Un}(X) \rightarrow$ $\operatorname{Un}\left(T_{P}^{0} C\right)$ which associates to a unipotent connection $\mathscr{V}$ on $X$ the residue connection $\operatorname{Res}_{P} \overline{\mathscr{V}}$ of the canonical logarithmic extension of $\mathscr{V}$. Using this construction we obtain more fibre functors $P$ which we now define:

Definition 26 Let $y \in T_{P}^{0} C\left(K_{v}\right)$ and let $\overline{\mathscr{V}}$ be a logarithmic connection on $C$ with logarithmic poles at $P$. Then the fibre functor $e_{y}: \operatorname{Un}(X) \rightarrow \operatorname{Vect}_{K_{v}}$ at $y$ is such that $\mathscr{V} \mapsto y^{*} \operatorname{Res}_{P} \overline{\mathscr{V}}$ where $\overline{\mathscr{V}}$ is the canonical logarithmic extension of $\mathscr{V}$.

The existence of a canonical Frobenius invariant de Rham path between any two points $x, y$ tangential or otherwise was demonstrated in [12, Theorem 4.1]. In loc.cit. Proposition 4.5 they identify the path $p_{x, y}$ from $x$ to $y$ for given points $x \in X_{v}\left(K_{v}\right)$ and $y \in T_{P}^{0} C\left(K_{v}\right)$ as being the constant term of a formal local solution to the differential equation defined by the logarithmic connection $\overline{\mathscr{V}}$. We now outline this construction in further detail.

There is a basis of global solutions to $\operatorname{Res}_{P} \overline{\mathscr{V}}=0$ with coefficients in $k[\log \bar{t}]$, with all solutions having the form $\exp \left(\operatorname{Res}_{P} \Omega \log \bar{t}\right) \cdot g$ with $g \in P^{* \bar{V}}$. The exponential will be finite since $\overline{\mathscr{V}}$ and hence $\operatorname{Res}_{P} \overline{\mathscr{V}}$ are unipotent. We can also find formal local horizontal solutions $s$ of $\overline{\mathscr{V}}$ near $P$ with coefficients in the ring $K_{v}[[t]][\log t]$. Here $\log t$ is treated as a formal variable with the property that $d \log t=d t / t$.

To analytically continue the horizontal section $s$ near $P$ to the tangent vector $\frac{d}{d t}$ we specialise to the fibre $P^{* \bar{V}} \otimes \mathscr{O}_{T_{P}^{0} C}$ by taking the constant term of this formal solution: that is, we let $t=\log t=0$. Call this constant term $s_{0}$. In Proposition 4.5, Besser and Furusho 
show that the path $p_{x, y}$ is the path $\left.\overline{\mathscr{V}} \mapsto\left(s \mapsto \exp \left(\operatorname{Res}_{P} C_{n}^{\prime} \log \bar{t}\right) \cdot s_{0}\right)\right)$. The specialisation of $\exp \left(\operatorname{Res}_{P} \Omega \log \bar{t}\right) \cdot s_{0}$ at $\bar{t}=1$ then is the analytic continuation along Frobenius of the horizontal section $s$ to the punctured tangent space, and this is precisely the constant term $s_{0}$. We recast this below as a computational algorithm:

Algorithm 6 (Computing iterated integrals with tangential basepoints on $X=C-D$ )

\section{Input}

- A smooth projective curve $C$ over a number field $K$ of characteristic 0 , a $K$-point $P$, $X:=C-\{P\}$ with affine model $f(x, y)=0$.

- A non-Archimedean valuation $v$ on $K$ and the completion $K_{v}$ of $K$ at $v$.

- A tangential basepoint $b$ and $x \in X_{v}\left(K_{v}\right)$.

- Differentials $\omega_{1}, \ldots, \omega_{n} \in \Omega_{C / K_{v}}^{1}(P)$ with at worst logarithmic poles at $P$.

\section{Output}

- The value of

$$
\int_{b}^{x} \omega_{1} \ldots \omega_{n} \in K_{v}
$$

\section{Algorithm}

I If $x \in] P[$ (the residue disk of $P$ ):

(1) Let $\partial_{b}$ be the derivation associated to $b$ and let $t$ be a local parameter at $P$ such that $\partial_{b} t=1$.

(2) Let $\sigma$ be a dummy variable and define

$$
\begin{aligned}
\varepsilon & =(x(\sigma), y(\sigma)) \\
z & =t(x) \\
\omega_{i}(t) & =f_{i}(t) d t
\end{aligned}
$$

where $f_{i}(t) d t$ is the Laurent expansion of $\omega_{i}$ at $P$ in $t$ for each $i$.

(3) Compute the formal iterated integral $\int_{\epsilon}^{x} \omega_{1} \ldots \omega_{n}$ :

$\int_{\sigma}^{z} f_{1}\left(t_{1}\right)\left(\int_{\sigma}^{t_{1}} f_{2}\left(t_{2}\right) \ldots\left(\int_{\sigma}^{t_{n-1}} f_{r-1}\left(t_{n-1}\right)\left(\int_{\sigma}^{t_{n}} f_{n}\left(t_{n}\right) d t_{n}\right) d t_{n-1}\right) \ldots d t_{2}\right) d t_{1}$

with output the logarithmic Coleman function

$$
a_{0}^{x}(\sigma)+a_{1}^{x}(\sigma) \log (\sigma)+a_{2}^{x}(\sigma) \log (\sigma)^{2}+\cdots
$$

where the $a_{i}^{x}(\sigma)$ analytic functions in the variable $\sigma$.

(4) Define

$$
\int_{b}^{x} \omega_{1} \ldots \omega_{n}:=a_{0}^{x}(0)
$$

i.e. set $\sigma=\log \sigma=0$ in the output of the previous step.

II Else $x \notin] P[$ :

(1) Choose $y \in] P[$ and do: 
(a) For $i=1, \ldots, n$ compute

$$
\int_{y}^{x} \omega_{1} \ldots \omega_{i}
$$

using Coleman integration.

(b) For $i=1, \ldots, n$ compute

$$
\int_{b}^{y} \omega_{i} \ldots \omega_{n}
$$

using Step I.

(2) Define

$$
\int_{b}^{x} \omega_{1} \ldots \omega_{n}:=\sum_{i=0}^{n} \int_{y}^{x} \omega_{1} \ldots \omega_{i} \int_{b}^{y} \omega_{i+1} \ldots \omega_{n}
$$

where the empty integral is defined to be 1 .

If we had chosen a different tangential basepoint $b^{\prime}$ then we need to make a different choice of normalising parameter $t^{\prime}$ with $\partial_{b^{\prime}} t^{\prime}=1$, where $\partial_{b^{\prime}}$ is the derivation associated to $b^{\prime}$. In [6, Lemma 3.2] it is shown that the above definition of the integral is independent of the choice of parameter $t^{\prime}$ satisfying this normalisation condition. For points $x$ lying outside ] $P$ [ we made use the following co-product formula for iterated integrals [2, Lemma 5.2.3]: if $x, y, z$ are points on $C$ such that that a path is to be taken from $x$ to $z$ through $y$ and $\omega_{1}, \ldots, \omega_{n}$ are holomorphic 1 -forms at these points then

$$
\int_{x}^{z} \omega_{1} \ldots \omega_{n}=\sum_{i=0}^{n} \int_{y}^{z} \omega_{1} \ldots \omega_{i} \int_{x}^{y} \omega_{i+1} \ldots \omega_{n}
$$

It is clear from the definition of the integral $\int_{b}^{x} \omega_{1} \ldots \omega_{n}$ that the co-product formula above still holds even if one of the endpoints of the path is a tangent vector. Hence, if we want to compute $\int_{b}^{x} \omega_{1} \ldots \omega_{n}$ with $x$ outside of $] P$ [ we find a point $\left.y \in\right] P$ [ and split the path at $y$. The co-product formula then gives us integrals of the form

$$
\int_{y}^{x} \omega_{1} \ldots \omega_{i}, \int_{b}^{y} \omega_{i+1} \ldots \omega_{n}
$$

where the first integral is computed using the algorithms in loc.cit. and the second integral is computed using Algorithm 6. Note also that if the differentials $\omega_{i}$ are regular also at $P$ then in fact the above algorithm simply gives us

$$
\int_{P}^{x} \omega_{1} \ldots \omega_{n}
$$

We wish to make applications of this to computing the de Rham period maps. If we replace the rational basepoint $b$ with a tangential basepoint we obtain a de Rham path space $\pi_{1, d r}(X ; b, x)=\operatorname{Isom}^{\otimes}\left(e_{b}, e_{x}\right)$ for $x \in X_{v}\left(K_{v}\right)$. This has a Hodge structure which is a limit Hodge structure of that on $\pi_{1, d r}(X ; y, x)$ as $y$ varies over $X\left(K_{v}\right)$ ([24]). As noted in loc.cit. it is sufficient to compute the Hodge filtration at rational basepoints.

Instead, we need to consider the logarithmic connection $\overline{\mathscr{U}}_{n}$ near $\infty$ : the restriction to the open $Y$ is $\mathscr{U}_{n}^{\prime}$ which is a logarithmic connection with connection matrix which can be computed using the algorithms of Sect. 3 . Near $\infty$ we can compute the parallel transport of $1 \in b^{*} \operatorname{Res}_{\infty} \overline{\mathscr{U}}_{n}$ to $x^{*} \overline{\mathscr{U}}_{n}$ for an $x \in Y$ using Proposition 4.5 in loc.cit. as described above. 
Let us consider how we do this in practice. Assume for now that $C$ is an elliptic curve or odd hyperelliptic curve, that $P$ is the point at infinity $\infty$ and fix a branch of the $p$-adic logarithm. The connection $\mathscr{U}_{n}^{\prime}$ is given by $\nabla^{\prime}=d+C_{n}^{\prime}$ on $Y$ as computed by Algorithm 3 . This connection matrix then defines an iterated integral which gives the parallel transport of $1 \in b^{*} \operatorname{Res}_{\infty} \overline{\mathscr{U}}_{n}$ to $x^{*} \overline{\mathscr{U}}_{n}$ as before. By computing a decomposition into a product of the Hodge trivialisation and a trivialisation of $F^{0} U_{n}^{d r} \backslash U_{n}^{d r}$ we may then describe the coordinates of the map $j_{n}^{d r}$ by an iterated integral with tangential basepoint. Finally we use Algorithm 6 to compute the image of this map for $x \in X_{v}\left(K_{v}\right)$.

\subsection{The levels 2 and 3 period maps on elliptic curves}

In this section we provide some explicit examples of the period maps on elliptic curves when the basepoint is tangential. First we consider the level 2 map which was considered in [10]. Throughout this section recall that we take $F \in K(C)$ with a simple pole at $\infty$ such that $d F-\alpha_{1}$ is regular at $\infty$.

Proposition 15 With $b$ a tangential basepoint at $\infty$ on the elliptic curve $X$, the level 2 unipotent Albanese map $j_{2}^{d r}: X_{v}\left(K_{v}\right) \rightarrow F^{0} U_{2}^{d r} \backslash U_{2}^{d r}$ on points $x \in X_{v}\left(K_{v}\right) \cap Y_{v}\left(K_{v}\right)$ is given by

$$
x \mapsto \int_{b}^{x} \alpha_{0} A_{0}+\int_{b}^{x}\left(F \alpha_{0}+\alpha_{0} \alpha_{1}^{\prime}\right)\left[A_{0}, A_{1}\right]
$$

where $\alpha_{1}^{\prime}=\alpha_{1}-d F$.

Proof In Proposition 4 we computed that the connection matrix of logarithmic extension $\overline{\mathscr{U}}_{2}$ over $Y$ can be taken to be

$$
C_{2}^{\prime}=\left(\begin{array}{ccccccc}
0 & 0 & 0 & 0 & -\alpha_{0} & 0 & 0 \\
0 & 0 & 0 & 0 & 0 & -\alpha_{0} & -F \alpha_{0} \\
0 & 0 & 0 & 0 & -\alpha_{1}^{\prime} & 0 & F \alpha_{0} \\
0 & 0 & 0 & 0 & 0 & -\alpha_{1}^{\prime} & 0 \\
0 & 0 & 0 & 0 & 0 & 0 & -\alpha_{0} \\
0 & 0 & 0 & 0 & 0 & 0 & -\alpha_{1}^{\prime} \\
0 & 0 & 0 & 0 & 0 & 0 & 0
\end{array}\right)
$$

where $\alpha_{1}^{\prime}=\alpha_{1}-d F$ for $F \in K(C)$ with a simple pole at $\infty$ such that $\alpha_{1}^{\prime}$ has at most a simple pole there also. Let $t$ be a local parameter at $\infty$. The parallel transport map from 1 at $b=\frac{d}{d t}$ to $x \in Y_{v}\left(K_{v}\right)$ then is given by

$$
\begin{aligned}
p_{2}^{c r}(x)= & +\int_{b}^{x} \alpha_{0} A_{0}+\int_{b}^{x} \alpha_{1}^{\prime} A_{1}+\int_{b}^{x} \alpha_{0} \alpha_{0} A_{0} A_{0}+\int_{b}^{x}\left(F \alpha_{0}+\alpha_{0} \alpha_{1}^{\prime}\right) A_{0} A_{1} \\
& +\int_{b}^{x}\left(\alpha_{1}^{\prime} \alpha_{0}-F \alpha_{0}\right) A_{1} A_{0}+\int_{b}^{x} \alpha_{1}^{\prime} \alpha_{1}^{\prime} A_{1} A_{1} \\
=1 & +\int_{b}^{x} \alpha_{0} A_{0}+\int_{b}^{x} \alpha_{1}^{\prime} A_{1}+\frac{1}{2}\left(\int_{b}^{x} \alpha_{0}\right)^{2} A_{0}^{2}+\int_{b}^{x} \alpha_{0} \alpha_{1}^{\prime}\left[A_{0}, A_{1}\right] \\
& +\int_{b}^{x}\left(\alpha_{1}^{\prime} \alpha_{0}+\alpha_{0} \alpha_{1}^{\prime}\right) A_{1} A_{0}+\int_{b}^{x} F \alpha_{0}\left[A_{0}, A_{1}\right]+\frac{1}{2}\left(\int_{b}^{x} \alpha_{1}^{\prime}\right)^{2} A_{1}^{2} \\
=1 & +\int_{b}^{x} \alpha_{0} A_{0}+\int_{b}^{x} \alpha_{1}^{\prime} A_{1}+\frac{1}{2}\left(\int_{b}^{x} \alpha_{0}\right)^{2} A_{0}^{2}+\int_{b}^{x}\left(F \alpha_{0}+\alpha_{0} \alpha_{1}^{\prime}\right)\left[A_{0}, A_{1}\right]
\end{aligned}
$$




$$
\begin{aligned}
& +\int_{b}^{x} \alpha_{1}^{\prime} \int_{b}^{x} \alpha_{0} A_{1} A_{0}+\frac{1}{2}\left(\int_{b}^{x} \alpha_{1}^{\prime}\right)^{2} A_{1}^{2} \\
= & \exp \left(\int_{b}^{x} \alpha_{1}^{\prime} A_{1}\right) \exp \left(\int_{b}^{x} \alpha_{0} A_{0}+\int_{b}^{x}\left(F \alpha_{0}+\alpha_{0} \alpha_{1}^{\prime}\right)\left[A_{0}, A_{1}\right]\right)
\end{aligned}
$$

Recall that we computed the Hodge filtration on $\overline{\mathscr{U}}_{2}$ over $Y$ to be generated by $1, A_{1}, A_{1}^{2}$. Hence we deduce that

$$
j_{2}^{d r}(x)=\int_{b}^{x} \alpha_{0} A_{0}+\int_{b}^{x}\left(F \alpha_{0}+\alpha_{0} \alpha_{1}^{\prime}\right)\left[A_{0}, A_{1}\right]
$$

for $x \in X_{v}\left(K_{v}\right) \cap Y_{v}\left(K_{v}\right)$.

Remark 33 To define the map $j_{2}^{d r}$ at points $x \in X_{v}\left(K_{v}\right)-Y_{v}\left(K_{v}\right)$ with tangential basepoint $b$ we proceed as follows: first we need to compute $p_{2}^{c r}(x)$. Recall that $p_{n}^{c r}$ is the parallel transport from $b$ to $x$ under $\overline{\mathscr{U}}_{n}$ so we are looking for a horizontal section $s$ which is the analytic continuation from $1 \in b^{*} \operatorname{Res}_{P} \overline{\mathscr{U}}_{n}$. Fix some $z \in Y_{v}\left(K_{v}\right) \cap X_{v}\left(K_{v}\right)$ and compute $p_{2}^{c r}(z)$ which is an element of $z^{*} \mathscr{U}_{2}^{\prime}$. Compute $\left(G_{2}^{-1}\right)_{Z} p_{2}^{c r}(z)=s_{z}$. Given this initial condition for $s$ over $X$, we find that $p_{2}^{c r}(x)$ is given by

$$
\left(G_{2}^{-1}\right)_{z} p_{2}^{c r}(z)+\sum_{|w| \leq 2} \int_{z}^{x} \alpha_{w} w
$$

We then compute $j_{2}^{d r}(x)$ as before by expressing $p_{2}^{c r}(x)=p^{H}(x) u_{2}(x)$ and putting $j_{2}^{d r}(x)=u_{2}(x)$.

We now move onto a more complicated example at level 3 but the principle here is the same.

Proposition 16 With $b$ a tangential basepoint at $\infty$ on the elliptic curve $X$, the level 3 unipotent Albanese map $j_{3}^{d r}: X_{v}\left(K_{v}\right) \rightarrow F^{0} U_{3}^{d r} \backslash U_{3}^{d r}$ on points $x \in X_{v}\left(K_{v}\right) \cap Y_{v}\left(K_{v}\right)$ is given by

$$
\begin{aligned}
x & \mapsto \int_{b}^{x} \alpha_{0} A_{0}+\int_{b}^{x}\left(\alpha_{0} \alpha_{1}^{\prime}+F \alpha_{0}\right)\left[A_{0}, A_{1}\right] \\
& +\frac{1}{2} \int_{b}^{x}\left(\alpha_{0} \alpha_{1}^{\prime} \alpha_{0}+F \alpha_{0} \cdot \alpha_{0}-\alpha_{0} \cdot F \alpha_{0}\right)\left[A_{0},\left[A_{1}, A_{0}\right]\right] \\
& +\int_{b}^{x}\left(\alpha_{0} \alpha_{1}^{\prime} \alpha_{1}^{\prime}+F \alpha_{0} \cdot \alpha_{1}^{\prime}+\alpha_{0}^{\prime}-\lambda \alpha_{1}^{\prime}\right)\left[\left[A_{0}, A_{1}\right], A_{1}\right]
\end{aligned}
$$

where $\alpha_{0}^{\prime}=\frac{1}{2} F^{2} \alpha_{0}-\lambda d F$ and $\alpha_{1}^{\prime}=\alpha_{1}-d F$.

Proof First we note that with the previously computed gauge transformation $G_{3}$ as computed in Proposition 5:

$$
C_{3}^{\prime}=\left(\begin{array}{cc}
0_{4} & D_{3}^{\prime} \\
0_{3 \times 4} & C_{2}^{\prime}
\end{array}\right), \quad D_{3}^{\prime}=\left(\begin{array}{ccccccc}
-\alpha_{0} & 0 & 0 & 0 & 0 & 0 & 0 \\
0 & -\alpha_{0} & 0 & 0 & 0 & 0 & 0 \\
0 & 0 & -\alpha_{0} & 0 & -F \alpha_{0} & 0 & 0 \\
0 & 0 & 0 & -\alpha_{0} & 0 & -F \alpha_{0} & -\alpha_{0}^{\prime} \\
-\alpha_{1}^{\prime} & 0 & 0 & 0 & F \alpha_{0} & 0 & 0 \\
0 & -\alpha_{1}^{\prime} & 0 & 0 & 0 & F \alpha_{0} & 2 \alpha_{0}^{\prime} \\
0 & 0 & -\alpha_{1}^{\prime} & 0 & 0 & 0 & -\alpha_{0}^{\prime} \\
0 & 0 & 0 & -\alpha_{1}^{\prime} & 0 & 0 & 0
\end{array}\right)
$$


where $F$ is as in the proof of Proposition 15. Therefore, the parallel transport of 1 on $b^{*} \operatorname{Res}_{\infty} \mathscr{U}_{3}$ to $x^{*} \mathscr{U}_{3}^{\prime}$ for $x \in Y_{v}\left(K_{v}\right)$ is given by

$$
\begin{aligned}
p_{3}^{c r}(x)= & +\sum_{|w| \leq 3} \int_{b}^{x} \alpha_{w} w+\int_{b}^{x} F \alpha_{0}\left(A_{0} A_{1}-A_{1} A_{0}\right) \\
& +\int_{b}^{x} \alpha_{0}^{\prime}\left(A_{0} A_{1} A_{1}-2 A_{1} A_{0} A_{1}+A_{1} A_{1} A_{0}\right) \\
& +\int_{b}^{x} F \alpha_{0} \cdot \alpha_{0}\left(A_{0} A_{1} A_{0}-A_{1} A_{0} A_{0}\right)+\int_{b}^{x} \alpha_{0} \cdot F \alpha_{0}\left(A_{0} A_{0} A_{1}-A_{0} A_{1} A_{0}\right) \\
& +\int_{b}^{x} F \alpha_{0} \cdot \alpha_{1}^{\prime}\left(A_{0} A_{1} A_{1}-A_{1} A_{0} A_{1}\right)+\int_{b}^{x} \alpha_{1}^{\prime} \cdot F \alpha_{0}\left(A_{1} A_{0} A_{1}-A_{1} A_{1} A_{0}\right) .
\end{aligned}
$$

We find that

$$
\begin{aligned}
\int_{b}^{x} & \alpha_{0} \cdot F \alpha_{0}\left(A_{0} A_{0} A_{1}-A_{0} A_{1} A_{0}\right)+\int_{b}^{x} F \alpha_{0} \cdot \alpha_{0}\left(A_{0} A_{1} A_{0}-A_{1} A_{0} A_{0}\right) \\
= & \frac{1}{2} \int_{b}^{x} \alpha_{0} \cdot F \alpha_{0}\left[A_{0},\left[A_{0}, A_{1}\right]\right]+\frac{1}{2} \int_{b}^{x} F \alpha_{0} \cdot \alpha_{0}\left[\left[A_{0}, A_{1}\right], A_{0}\right] \\
& +\frac{1}{2} \int_{b}^{x} F \alpha_{0} \int_{b}^{x} \alpha_{0}\left(\left[A_{0}, A_{1}\right] A_{0}+A_{0}\left[A_{0}, A_{1}\right]\right) .
\end{aligned}
$$

Observe also that

$$
\begin{gathered}
\int_{b}^{x} F \alpha_{0} \cdot \alpha_{1}^{\prime}\left(A_{0} A_{1} A_{1}-A_{1} A_{0} A_{1}\right)+\int_{b}^{x} \alpha_{1}^{\prime} \cdot F \alpha_{0}\left(A_{1} A_{0} A_{1}-A_{1} A_{1} A_{0}\right) \\
=\int_{b}^{x} F \alpha_{0} \cdot \alpha_{1}^{\prime}\left[\left[A_{0}, A_{1}\right], A_{1}\right]+\int_{b}^{x} \alpha_{1}^{\prime} \int_{b}^{x} F \alpha_{0} A_{1}\left[A_{0}, A_{1}\right] .
\end{gathered}
$$

Putting these formulations together and using the approach that we have previously taken we find that

$$
p_{3}^{c r}(x)=\exp \left(\int_{b}^{x} \alpha_{1}^{\prime}\left(A_{1}+\lambda\left[\left[A_{0}, A_{1}\right], A_{1}\right]\right) \exp \left(u_{3}(x)\right)\right.
$$

where $u_{3}(x)$ is as in the statement of the proposition. To define $j_{3}^{d r}$ at points not in $Y_{v}\left(K_{v}\right)$ we proceed as before.

\subsection{The level 2 period map on hyperelliptic curves}

We again consider a genus $g$ odd hyperelliptic curve $C$ and determine the level 2 unipotent Albanese map on $X:=C-\{\infty\}$ with tangential basepoint following the approach used above.

Proposition 17 With $b$ a tangential basepoint at $\infty$ on an odd affine hyperelliptic curve $X$ of genus $g$ the level 2 unipotent Albanese map $j_{2}^{d r}: X_{v}\left(K_{v}\right) \rightarrow F^{0} U_{2}^{d r} \backslash U_{2}^{d r}$ on points $x \in X_{v}\left(K_{v}\right) \cap Y_{v}\left(K_{v}\right)$ is given by 


$$
\begin{aligned}
j_{2}^{d r}(x)= & \sum_{k=0}^{g-1} \int_{b}^{x} \alpha_{k} A_{k}+\frac{1}{2} \sum_{k, l=0}^{g-1} \int_{b}^{x} \alpha_{k} \alpha_{l}\left[A_{k}, A_{l}\right] \\
& +\sum_{k=0}^{g-1} \sum_{l=g}^{2 g-1} \int_{b}^{x}\left(\alpha_{k} \alpha_{l}^{\prime}+c_{2}^{2 g l+k+1,0}\right)\left[A_{k}, A_{l}\right] \\
& +\sum_{k=g}^{2 g-1} \sum_{\substack{0 \leq i<g \\
g \leq j<2 g}} c_{i j k} \int_{b}^{x} \alpha_{k}^{\prime}\left[A_{j}, A_{i}\right]
\end{aligned}
$$

Proof We will find after application of Algorithm 3 that

$$
\begin{aligned}
p_{2}^{c r}(x)= & +\sum_{k=0}^{g-1} \int_{b}^{x} \alpha_{k} A_{k}+\sum_{k=g}^{2 g-1} \int_{b}^{x} \alpha_{k}^{\prime} A_{k}+\sum_{k=0}^{g-1} \sum_{l=g}^{2 g-1} \int_{b}^{x} \alpha_{k} \alpha_{l}^{\prime} A_{k} A_{l} \\
& +\sum_{k=g}^{2 g-1} \sum_{l=0}^{g-1} \int_{b}^{x} \alpha_{k}^{\prime} \alpha_{l} A_{k} A_{l}+\sum_{k=g}^{2 g-1} \sum_{l=g}^{2 g-1} \int_{b}^{x} \alpha_{k}^{\prime} \alpha_{l}^{\prime} A_{k} A_{l} \\
& -\sum_{k=0}^{2 g-1} \sum_{l=0}^{2 g-1} \int_{b}^{x} c_{2}^{2 g k+l+1,0} A_{k} A_{l}
\end{aligned}
$$

where $\alpha_{k}^{\prime}=\alpha_{k}-d h_{1}^{k+1,0}$ and $c_{2}^{2 g k+l+1,0}$ are 1-forms on $C$ with at worst logarithmic poles at $\infty$. The computations in the proof of Proposition 10 show that we can take $c_{2}^{2 g k+l+1,0}=0$ when $k, l<g$ and otherwise $c_{2}^{2 g k+l+1,0}=c_{2}^{2 g l+k+1,0}$. Now the extra expression appearing in $p_{2}^{c r}(x)$

$$
-\sum_{k=0}^{2 g-1} \sum_{l=0}^{2 g-1} \int_{b}^{x} c_{2}^{2 g k+l+1,0} A_{k} A_{l}
$$

can be rewritten as

$$
\begin{aligned}
& -\sum_{k=0}^{g-1} \sum_{l=g}^{2 g-1} \int_{b}^{x} c_{2}^{2 g k+l+1,0}\left[A_{k}, A_{l}\right]-\frac{1}{2} \sum_{k, l=g}^{2 g-1} \int_{b}^{x} c_{2}^{2 g k+l+1,0}\left[A_{k}, A_{l}\right] \\
& =\sum_{k=0}^{g-1} \sum_{l=g}^{2 g-1} \int_{b}^{x} c_{2}^{2 g l+k+1,0}\left[A_{k}, A_{l}\right]+\frac{1}{2} \sum_{k, l=g}^{2 g-1} \int_{b}^{x} c_{2}^{2 g l+k+1,0}\left[A_{k}, A_{l}\right] .
\end{aligned}
$$

We can then conclude by noting that

$$
\begin{aligned}
p_{2}^{c r}(x)=\exp & \left(\sum_{k=g}^{2 g-1} \int_{b}^{x} \alpha_{k}^{\prime}\left(A_{k}+\sum_{\substack{0 \leq i<g \\
g \leq j<2 g}} a_{1, k+1}^{2,1+j+i(2 g)}\left[A_{i}, A_{j}\right]\right)\right. \\
& \left.+\frac{1}{2} \sum_{l, k=g}^{2 g-1} \int_{b}^{x} \alpha_{k}^{\prime} \alpha_{l}^{\prime}\left[A_{k}, A_{l}\right]\right)
\end{aligned}
$$




$$
\begin{aligned}
& \times \exp \left(\tilde{u}_{2}(x)\right) \exp \left(\sum_{k=0}^{g-1} \sum_{l=g}^{2 g-1} \int_{b}^{x} c_{2}^{2 g l+k+1,0}\left[A_{k}, A_{l}\right]\right) \\
& \exp \left(\frac{1}{2} \sum_{k, l=g}^{2 g-1} \int_{b}^{x} c_{2}^{2 g l+k+1,0}\left[A_{k}, A_{l}\right]\right)
\end{aligned}
$$

where

$$
\begin{aligned}
\tilde{u}_{2}(x)= & \sum_{k=0}^{g-1} \int_{b}^{x} \alpha_{k} A_{k}+\frac{1}{2} \sum_{k, l=0}^{g-1} \int_{b}^{x} \alpha_{k} \alpha_{l}\left[A_{k}, A_{l}\right]+\sum_{k=0}^{g-1} \sum_{l=g}^{2 g-1} \int_{b}^{x} \alpha_{k} \alpha_{l}^{\prime}\left[A_{k}, A_{l}\right] \\
& +\sum_{k=g}^{2 g-1} \sum_{\substack{0 \leq i<g \\
g \leq j<2 g}} a_{1, k+1}^{2,1+j+i(2 g)} \int_{b}^{x} \alpha_{k}^{\prime}\left[A_{j}, A_{i}\right] .
\end{aligned}
$$

Since $\sum_{k=0}^{g-1} \sum_{l=g}^{2 g-1} \int_{b}^{x} c_{2}^{2 g l+k+1,0}\left[A_{k}, A_{l}\right]$ and $\frac{1}{2} \sum_{k, l=g}^{2 g-1} \int_{b}^{x} c_{2}^{2 g l+k+1,0}\left[A_{k}, A_{l}\right]$ are expressions in words of length 2 the last two exponentials above commute with all others. Since $\left[A_{k}, A_{l}\right]$ is a generator of $F^{0} \mathscr{U}_{2}$ for $k, l \geq g$ then we can conclude that $j_{2}^{d r}(x)$ is as in the statement of the proposition.

Acknowledgements The author would like to thank Minhyong Kim for introducing him to the non-abelian Chabauty method, for the suggestion of this problem, and for his help and encouragement. This work was completed while the author was an EPSRC (Grant No. EP/M50659X/1) funded D.Phil. student (Award No. MATH1503) at the Mathematical Institute, University of Oxford. He would also like to thank the referree for many helpful comments.

Open Access This article is licensed under a Creative Commons Attribution 4.0 International License, which permits use, sharing, adaptation, distribution and reproduction in any medium or format, as long as you give appropriate credit to the original author(s) and the source, provide a link to the Creative Commons licence, and indicate if changes were made. The images or other third party material in this article are included in the article's Creative Commons licence, unless indicated otherwise in a credit line to the material. If material is not included in the article's Creative Commons licence and your intended use is not permitted by statutory regulation or exceeds the permitted use, you will need to obtain permission directly from the copyright holder. To view a copy of this licence, visit http://creativecommons.org/licenses/by/4.0/.

\section{Index of notation}

This index lists the notation used throughout the paper for ease of reference.

$\begin{array}{llll}(\mathscr{U}, u), 6 & D_{n+1}^{\prime}, 17 & H_{n+1}^{r, j, i}, 20 & P^{d r}(x), 8 \\ (\mathscr{V}, \nabla), 5 & D_{n+1}, 16 & H_{n+1}, 17 & P_{n}^{d r}(x), 8 \\ \left(\mathscr{V}, \nabla, F^{\bullet}\right), 27 & F^{\bullet}, 7 & I_{m}, 32,40 & R, 7 \\ A_{i}, 7 & F^{\bullet} P^{d r}, 9 & K, 5 & R_{n}, 7 \\ C_{n+1}^{\prime}, 17 & F^{\bullet} \overline{\mathscr{U}}_{n}, 28,31 & K_{v}, 7 & S_{m}^{f}, 35 \\ C_{n}^{r, i}, 18 & G_{n+1}, 17 & P^{c r}(y), 9 & S_{m}^{f}, 33 \\ C_{n}, 16 & H_{n}^{r, i}, 18 & P^{d r}, 8 & T_{P}^{0} C, 50\end{array}$




$\begin{array}{llll}T_{m}^{f}, 35 & \infty, 15 & \left\{\left(\mathscr{U}_{n}, u_{n}\right)\right\}_{n \geq 0}, 6 & c_{n}^{r, i}, 18,20 \\ T_{m}^{f}, 32 & \operatorname{Res}_{P} \overline{\mathscr{V}}, 50 & \mathscr{C}^{*}, 6 & e_{b}, 6 \\ T_{P} C, 50 & \mathscr{U}, 6 & \mathscr{P}, 8 & h_{n}^{r, i}, 18,20 \\ U^{c r}, 9 & \overline{\mathscr{U}}, 12 & \nabla, 5,11 & j^{d r}, j_{n}^{d r}, 10,45,48 \\ U^{d r}, 8 & \overline{\mathscr{U}}_{n}, 12 & \phi, 18 & k, 7 \\ U_{n}^{d r}, 8 & \mathscr{U}_{n}, 7 & \psi, 18,19 & p_{n}^{H}(x), 10 \\ V_{d r}, 7 & \mathrm{Un}^{\prime}(X), 6 & \tau, 31 & p_{n}^{c r}(x), 10,45 \\ \mathscr{B}_{n}, 15,25,28 & \mathrm{Un}_{n}(X), 6 & \tilde{S}_{m}^{f}, 33 & t, 50 \\ \Delta, 8 & \alpha_{i}, 7 & \tilde{T}_{m}^{f}, 33 & u_{n}(x), 10 \\ \mathscr{F}_{n}, 30 & \int_{x}^{y} \omega_{1} . . \omega_{r}, 11 & a_{m, f}^{l, k}, 31,36 & v, 7 \\ \Omega_{C}^{1}(D), 11 & \left\{\left(\overline{\mathscr{U}}_{n}, u_{n}\right)\right\}, 12 & b_{m, f}^{l, k}, 32,36 & w_{l}^{k}, 15,25\end{array}$

\section{References}

1. Andreatta, F., Iovita, A., Kim, M.: A p-adic nonabelian criterion for good reduction of curves. Duke Math. J. 164(13), 2597-2642 (2015)

2. Balakrishnan, J.: Coleman Integration for Hyperelliptic Curves: Algorithms and Applications. ProQuest LLC, Ann Arbor, MI. Thesis (Ph.D.)-Massachusetts Institute of Technology (2011)

3. Balakrishnan, J., Bradshaw, R., Kedlaya, K.: Explicit Coleman integration for hyperelliptic curves. In: Algorithmic number theory, Lecture Notes in Comput. Sci., vol. 6197, pp. 16-31. Springer, Berlin (2010)

4. Balakrishnan, J., Dogra, N.: Quadratic Chabauty and rational points II: Generalised height functions on Selmer varieties (2016). ArXiv:1705.00401, preprint only - v1 accessed

5. Balakrishnan, J.S.: Iterated Coleman integration for hyperelliptic curves. In: ANTS X-Proceedings of the Tenth Algorithmic Number Theory Symposium, Open Book Ser., vol. 1, pp. 41-61. Math. Sci. Publ., Berkeley, CA (2013)

6. Balakrishnan, J.S., Besser, A.: Coleman-Gross height pairings and the $p$-adic sigma function. J. Reine Angew. Math. 698, 89-104 (2015)

7. Balakrishnan, J.S., Dogra, N.: An effective Chabauty-Kim theorem (2018). ArXiv:1803.10102, preprint only - v1 accessed

8. Balakrishnan, J.S., Dogra, N.: Quadratic Chabauty and rational points, I: $p$-adic heights. Duke Math. J. 167(11), 1981-2038 (2018). With an appendix by J. Steffen Müller

9. Balakrishnan, J.S., Dogra, N., Müller, J.S., Tuitman, J., Vonk, J.: Explicit Chabauty-Kim for the split cartan modular curve of level 13 (2018). ArXiv:1711.05846, preprint only - v1 accessed

10. Balakrishnan, J.S., Kedlaya, K.S., Kim, M.: Appendix and erratum to "Massey products for elliptic curves of rank 1" [mr2629986]. J. Am. Math. Soc. 24(1), 281-291 (2011)

11. Besser, A.: Coleman integration using the Tannakian formalism. Math. Ann. 322(1), 19-48 (2002)

12. Besser, A., Furusho, H.: The double shuffle relations for $p$-adic multiple zeta values. In: Primes and knots, Contemp. Math., vol. 416, pp. 9-29. Am. Math. Soc., Providence, RI (2006)

13. Chabauty, C.: Sur les points rationnels des courbes algébriques de genre supérieur à l'unité. C. R. Acad. Sci. Paris 212, 882-885 (1941)

14. Chiarellotto, B., Le Stum, B.: F-isocristaux unipotents. Composit. Math. 116(1), 81-110 (1999)

15. Coates, J., Kim, M.: Selmer varieties for curves with CM Jacobians. Kyoto J. Math. 50(4), 827-852 (2010)

16. Coleman, R.: Effective Chabauty. Duke Math. J. 52(3), 765-770 (1985)

17. Deligne, P.: Équations différentielles à points singuliers réguliers. Lecture Notes in Mathematics, vol. 163. Springer, Berlin-New York (1970)

18. Deligne, P., Illusie, L.: Relèvements modulo $p^{2}$ et décomposition du complexe de de Rham. Invent. Math. 89(2), 247-270 (1987)

19. Dogra, N.: Topics in the theory of Selmer varieties (2015). Thesis (Ph.D.)-University of Oxford

20. Ellenberg, J.S., Rayor Hast, D.: Rational points on solvable curves over $\mathbb{Q}$ via non-abelian chabauty. ArXiv e-prints (2017). ArXiv:1706.00525, preprint only-v2 accessed

21. Hadian, M.: Motivic fundamental groups and integral points. Duke Math. J. 160(3), 503-565 (2011) 
22. Kim, M.: The motivic fundamental group of $\mathbb{P}^{1} \backslash\{0,1, \infty\}$ and the theorem of Siegel. Invent. Math. 161(3), 629-656 (2005)

23. Kim, M.: The unipotent Albanese map and Selmer varieties for curves. Publ. Res. Inst. Math. Sci. 45(1), 89-133 (2009)

24. Kim, M.: Massey products for elliptic curves of rank 1. J. Am. Math. Soc. 23(3), 725-747 (2010)

25. Kim, M.: $p$-adic $L$-functions and Selmer varieties associated to elliptic curves with complex multiplication. Ann. Math. 172(1), 751-759 (2010)

26. Vologodsky, V.: Hodge structure on the fundamental group and its application to $p$-adic integration. Mosc. Math. J. 3(1), 205-247 (2003)

27. Wildeshaus, J.: Realizations of polylogarithms: lecture notes in mathematics. Springer, Berlin (1997)

28. Wojtkowiak, Z.: Cosimplicial objects in algebraic geometry. In: Algebraic $K$-theory and algebraic topology (Lake Louise, AB, 1991), NATO Adv. Sci. Inst. Ser. C Math. Phys. Sci., vol. 407, pp. 287-327. Kluwer Acad. Publ., Dordrecht (1993)

Publisher's Note Springer Nature remains neutral with regard to jurisdictional claims in published maps and institutional affiliations. 